TITLE:

\title{
Phase Separation and Dewetting in Polymer Blend Thin Films( Dissertation_全文)
}

$\operatorname{AUTHOR}(\mathrm{S}):$

Ogawa, Hiroki

CITATION:

Ogawa, Hiroki. Phase Separation and Dewetting in Polymer Blend Thin Films. 京都大学, 2008, 博士(工学)

ISSUE DATE:

2008-07-23

URL:

https://doi.org/10.14989/doctor.k14104

RIGHT: 
Phase Separation and Dewetting in Polymer Blend

\author{
Thin Films
}

Hiroki Ogawa

2008 
Chapter 1

General Introduction

1.1 Introduction 1

1.2 Theoretical Background 3

1.2.1 Spinodal Decomposition 3

1.2.2 Nucleation and Growth 11

$\begin{array}{lll}\text { 1.2.3 Dewetting } & 12\end{array}$

1.3 Current Situation of Polymer Blend Thin Film Studies 17

1.4 Outline of This Thesis 19

References $\quad 21$

Chapter 2

Basic Theories of Scattering and Reflectivity, and Experimental Techniques $\quad 25$

2.1 Introduction 25

2.2 Theory of Scattering and Reflectivity 25

$\begin{array}{ll}\text { 2.2.1 Scattering Theory } & 25\end{array}$

2.2.2 Reflectivity Theory 30

2.3 Experimental Apparatus 36

2.3.1 Small-Angle Light Scattering 36

2.3.1.1 Instrumental Setup $\quad 37$

2.3.1.2 Basic Performances $\quad 39$

2.3.2 Neutron Reflectometers 44

2.3.3 Optical Microscope 48

2.3.4 Atomic Force Microscope 48

2.3.5 Scanning Near-Field Optical Microscope $\quad 49$

References $\quad 50$

Chapter 3

Phase Separation and Dewetting in Polystyrene/Poly(vinyl methyl ether) Blend Thin Films in a Wide Thickness Range 51

3.1 Introduction 51

3.2 Experimental 51

3.3 Results and Discussion 52 
$\begin{array}{ll}3.4 \text { Conclusion } & 66\end{array}$

References $\quad 68$

Chaper 4

Phase Separation and Dewetting for Ultra-thin Films in Polystyrene/Poly(vinyl methyl ether) Blend $\quad 70$

$\begin{array}{ll}4.1 \text { Introduction } & 70\end{array}$

$\begin{array}{ll}4.2 \text { Experimental } & 70\end{array}$

$\begin{array}{ll}4.3 \text { Results and Discussion } & 71\end{array}$

4.4 Conclusion 85

References $\quad 86$

Chapter 5

Composition Fluctuations before Dewetting in Polystyrene/Poly(vinyl methyl $\begin{array}{lr}\text { ether) Blend Thin Films } & 87\end{array}$

$\begin{array}{ll}5.1 \text { Introduction } & 87\end{array}$

5.2 Experimental $\quad 87$

5.3 Results and Discussion $\quad 88$

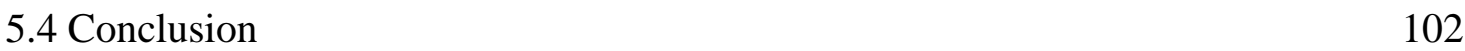

$\begin{array}{ll}\text { References } & 104\end{array}$

Chapter 6

Time-resolved Neutron Reflectivity Measurements for Dewettng Process in $\begin{array}{ll}\text { Polystyrene/Poly(vinyl methyl ether) Blend Thin Films } & 105\end{array}$

$\begin{array}{ll}6.1 \text { Introduction } & 105\end{array}$

$\begin{array}{ll}6.2 \text { Experimental } & 105\end{array}$

$\begin{array}{ll}\text { 6.3 Results and Discussion } & 106\end{array}$

$\begin{array}{ll}\text { 6.4 Conclusion } & 117\end{array}$

$\begin{array}{ll}\text { References } & 118\end{array}$

Chapter 7

Effect of Critical and Non-Critical Composition for Phase Separation and Dewetting in Polystyrene/Poly(vinyl methyl ether) Blend Thin Films 
$\begin{array}{ll}7.1 \text { Introduction } & 119\end{array}$

$\begin{array}{ll}7.2 \text { Experimental } & 119\end{array}$

$\begin{array}{ll}7.3 \text { Results and Discussion } & 120\end{array}$

$\begin{array}{ll}7.4 \text { Conclusion } & 127\end{array}$

$\begin{array}{ll}\text { References } & 129\end{array}$

$\begin{array}{lr}\text { Summary } & 130\end{array}$

$\begin{array}{ll}\text { List of Publications } & 133\end{array}$

$\begin{array}{ll}\text { Acknowledgement } & 134\end{array}$ 


\section{Chapter 1}

\section{General Introduction}

\subsection{Introduction}

Properties of polymer thin films on solid substrates are of great importance from scientific as well as industrial viewpoints because many important phenomena are related to the surface properties such as coating, adhesive, surface friction, lubricants, and dielectric layer [1-4]. Many studies on one component thin films have been carried out to reveal interesting but unusual properties of thin films. It was reported by many groups [5-9] that the glass transition temperature $T_{\mathrm{g}}$ decreased with film thickness below about $40 \mathrm{~nm}$, and the thermal expansivity of the thin films is also a big issue under debate in the thin film research field [8-13].

In addition, dewetting is also very important phenomenon in thin films from viewpoints of stability. A thin liquid film coated on a substrate, which itself may be either another liquid or a solid, may not be thermodynamically stable. If such thin film is prepared in this unstable state, it will subsequently break up into droplets. This process is dewetting, which is of importance in many areas of technology that involve thin films and coatings, microelectronics fabrication and surface pattering [14-16], and has been focus of considerable recent attention. It is now apparent that dewetting may occur by a number of different mechanisms, and theoretical and experimental studies have begun to clarify these mechanisms [17-23] and identify the factors controlling the dynamics of dewetting and the morphology of the resulting dewetted films on both solid and liquid substrates [24-26]. For very thin films dewetting may follow from an interfacial instability driven by dispersion forces, which amplify thermally excited capillary waves, either at the surface of liquid film, or at the interface between a liquid film and a liquid substrate. A wavelength selection mechanism leads to a final morphology characterized by a well-defined length scale, which is related to the wavelength of the capillary wave which grows the fastest in the early time stage, i. e., linear regime.

In contrast to single component, multi component systems confined to a thin film have received much less attention, in part, because confinement introduces new 
complications, such as surface and interface interactions. In polymer blends, the surface composition typically differs from the bulk because each component tends to have different surface energies. For a phase-separating thin film blend, the surface can form a wetting layer via diffusion (slow) of the surface preferred component or by hydrodynamic (fast) flow of this component through channels in the nonwet phase. Confinement can influence the thermodynamic and kinetic behavior in thin films. For example, surface interactions and/or confinement can shift the critical temperature, which in turn changes the driving force for phase separation [27-29]. The resulting segregation or wetting will break the compositional symmetry and can cause oscillatory compositional profiles perpendicular to the surface, a phenomenon called surface directed spinodal decomposition (SDSD) [30].

In order to understand the phase separation in polymer blend thin films, we have to know the phase separation processes in bulk.

The kinetics of ordering processes in a phase transition is a subject of many theoretical and experimental investigations in the field of small-molecule or atomic systems, such as fluid mixtures, metallic alloys, and inorganic glasses. Such a study is also attracting much theoretical and experimental interest in the field of polymers [31, 32]. The dynamics in the early stage of phase separation is investigated on the kinetics of first-order phase transition. There are two categories for the kinetics of the phase transition, the spinodal decomposition and nucleation and growth. The former corresponds to the phase separation from the unstable state where the thermal fluctuations grow from the infinite minimal perturbations, and the latter corresponds to the phase transition from metastable state where formation of the critical nucleus having a finite size should observed. Cahn et al. [33] formalized the relationship between fluctuation and thermodynamical stability. This Chan's linealized theory, which will be explained in Chapter 2, describes the early stage of time evolution of phase transition but cannot be applied to the late stage. In the late stage, the fluctuations produce the so-called clusters which grow with time. It has been found that a dynamical scaling law proposed by Furukawa [34] fairly well describes the time evolution of the structure function in the late stage. These theories can describe the dynamics of the phase transition for the system having first order parameter such as density.

In studies of polymer blend thin films, attention has focussed on the effect that surfaces have on microstructure evolution within the film during phase separation. It 
is typically found that segregated layers parallel to the surfaces develop which persist some distance into the film. This process, known as SDSD, is reasonably well understood both experimentally [35-38] and theoretically [39-41]. In recent years, spinodal decomposition morphology of binary polymer blend thin films has received significant attention [42-52]. These studies reveal that films can display a variety of structures, bi-continuous and discrete phases, and stratified or perforated layers. This rich diversity of morphologies results from the dynamics interplay between a variety factors including phase separation, wetting, hydrodynamics, capillary fluctuations, dewetting and confinement. Although an understanding of the processes of both dewetting and phase separation is well advanced, the coupling between the two has received little attention theoretically. Recently Clarke et al. investigated this problem from purely thermodynamic viewpoint [53-54], but they concluded that their theory and results are not directly comparable to the experimental results of Composto et al [83-87].

A comprehensive understanding of the morphology evolution in multi component thin film is lacking because of many factors involved, such as the interplay between factors and the range of parameter space. In particular, the dynamic interplay between factors makes it difficult to isolate the role of each factor on the morphology evolution. For example, dewetting and phase separation occur simultaneously and perturb each other. On the other hand, these factors depend differently on specific parameters, such as temperature, composition, film thickness, viscosity, surface and interfacial interactions. As such, a comprehensive understanding of phase separation as well as dewetting in thin films requires systematic studies to unravel the effect of each factor on the morphology evolution. In this thesis, therefore, we investigated morphology and kinetics of phase separation as well as dewetting in polymer blend thin films as a function of one parameter, film thickness, temperature, composition, while keeping others fixed. In the following sections we briefly review the mechanism of phase separation: nucleation and growth and spinodal decomposition, and also dewetting. We will describe current situation and also motivation of this studies in section 1.3 and outline of this thesis in section 1.4. 


\subsection{Theoretical Background}

\subsubsection{Spinodal Decomposition (SD)}

In this section, we briefly review the theories which we shall use for the analysis of our experimental data. A good review of the theories was given by Gunton, San Miguel and Sahni [55]. The time-evolution of the conserved order parameter $S(\boldsymbol{r}, t)$ is generally given by the nonlinear Langevin equation.

$$
\frac{\partial S(\boldsymbol{r}, t)}{\partial t}=L \nabla^{2} \frac{\delta \Phi(t)}{\delta S(\boldsymbol{r}, t)}+f_{T}(\boldsymbol{r}, t)
$$

where $f_{T}(\boldsymbol{r}, t)$ describes the effects of the random thermal force [56], $L$ is the kinetics Onsager coefficient, and $\frac{\delta \Phi(t)}{\delta S(\boldsymbol{r}, t)}$ is the local chemical potential. The potential $\Phi(t)$ may be given by the Ginzburg-Landau expansion near the critical point and for the early stage of unmixing.

$$
\Phi(t)=\int d \boldsymbol{r}\left\{\frac{1}{2}[\nabla S(\boldsymbol{r}, t)]^{2}-\frac{1}{2} h S(\boldsymbol{r}, t)^{2}+\frac{1}{4 !} g S(\boldsymbol{r}, t)\right\}^{4}
$$

Combining Eq. (1.1) and (1.2) one obtains,

$$
\frac{\partial S(\boldsymbol{r}, t)}{\partial t}=-L \nabla^{2}\left\{\left(h+\nabla^{2}\right) S-\frac{1}{6} g S^{3}\right\}+f_{T}(\boldsymbol{r}, t)
$$

Equation 1.3 is known as time-dependent Ginzgurg-Landau equation (TDGL) which gives the general principle to describe spinodal decomposition in solids such as metallic alloys and inorganic glasses. The equation may be applied for unmixing kinetics of fluid mixtures in early stage, where the hydrodynamics effect [57] does not significantly contribute to the ordering process and of liquid polymer mixtures, also in early stage.

Thus the ordering process is intrinsically nonlinear $(g \neq 0)$ and nonlocal $(\nabla S \neq 0)$ as shown in Eq. (1.3). However one can simplify the TDGL by neglecting the $g S^{3}$ term in Eq. (1.3), which may be legitimate in the early stage, since $S$ is small, and by neglecting $f_{T}(\boldsymbol{r}, t)$, which also may be legitimate, if the effects of the thermal fluctuation force are small compared with that of the diffusion flux $L \nabla^{2}\{\delta \Phi(t) / \delta S(\boldsymbol{r}, t)\}$. Then one obtains the linear time-evolution equation for the order parameter, as first given by Cahn [58],

$$
\frac{\delta S(\boldsymbol{r}, t)}{\delta t}=-L \nabla^{2}\left(\nabla^{2}+h\right) S(\boldsymbol{r}, t)
$$


The linearized time-evolution equation gives the equation of motion for the $q$-Fourier component of the order parameter $S_{q}(t)$ as given by

$$
d S_{q}(t) / d t=L q^{2}\left(h-q^{2}\right) S_{q}(t)
$$

and hence the exponential growth behavior

$$
S_{q}(t)=S_{q}(0) \exp [R(q) t]
$$

with

$$
R(q)=L q^{2}\left(h-q^{2}\right)
$$

The expression $R(q)$ for the growth rate of the $q$-Fourier mode is identical to that obtained by Cahn [58] with the following substitution:

$$
\begin{aligned}
& L=2 M \kappa \\
& h=-\left(\partial^{2} f / \partial \phi_{2}\right) /(2 \kappa)
\end{aligned}
$$

Where $M$ is the mobility constant, $\kappa$ is the coefficient associated with gradient free energy density $\kappa(\nabla \phi)^{2}, f$ is the free energy density of a mixture, and $\phi$ is the concentration of one component in the binary mixture.

The linearized theory of the spinodal decomposition (SD) was also developed for polymer systems, and $\kappa$ and $f$ were calculated by van Aartsen [59] for symmetric polymer mixtures (polymer $A$ +polymer $B$ ). Pincus [60] extended and corrected deGennes' theory on the $q$-dependent of the Onsager coefficient $L(q)$. Binder [61] refined further the linearized theory in which $q$-dependent of the Onsager coefficient and the thermodynamic driving force as well as the thermal fluctuating forces are given for asymmetric polymer pairs, ie., a polymer pair having different polymerization indices $N_{A}$ and $N_{B}$, Kuhn statistical segment length $a_{A}$ and $a_{B}$, and selfdiffusivities $D_{A}$ and $D_{B}$.

In this article we shall consider unmixing processes in the small $q$ regime

$$
q<<1 / R_{g K} \quad(K=A \text { or } B)
$$

as can be studied by light-scattering experiments. The equation $R_{g K}$ is the radius of gyration of $K$-polymer,

$$
R_{g K}=N_{K} a_{K}^{2} / 6
$$

where $N_{K}$ is the polmerization index. The growth rate $R(\boldsymbol{q})$ for the asymmetric polymer pairs in the small $\boldsymbol{q}$-regime is given by

$$
R(q)=q^{2} D_{\text {app }}\left[1-q^{2} /\left(2 q_{m}^{2}\right)\right]
$$

on the basis of the linearized theory, where $D_{a p p}$ is the mutual (or apparent) diffusion coefficient and $q_{m}$ is the wavelength of the particular Fourier component of 
fluctuations with a maximum growth rate.

$$
\begin{aligned}
& D_{a p p}=D_{A} D_{B} N\left[D_{A} N_{A} \phi_{A}+D_{B} N_{B} \phi_{B}\right]^{-1} \varepsilon \\
& q_{m}^{2}=9 \bar{N}\left(N_{A} N_{B} \bar{a}^{2}\right)^{-1} \varepsilon
\end{aligned}
$$

with

$$
\begin{aligned}
& \varepsilon=\left(\chi-\chi_{S}\right) / \chi_{S}=\frac{1}{\chi_{S}}\left(\frac{\partial \chi}{\partial T}\right)_{T_{S}} \Delta T_{S}+\cdots \\
& 2 \chi_{S}=\left(\phi N_{A}\right)^{-1}+\left[(1-\phi) N_{B}\right]^{-1} \\
& \bar{N}=\phi N_{A}+(1-\phi) N_{B} \\
& \bar{a}^{2}=a_{A}^{2}(1-\phi)+a_{B}^{2} \phi
\end{aligned}
$$

where $\varepsilon$ is the thermodynamics driving force for $\mathrm{SD}, \chi$ is the Flory interaction parameter between $A$ and $B$ [62], and $\chi_{s}$ is the $\chi$-parameter at the spinodal point.

For symmetric polymer pairs with $N_{A}=N_{B}=N, a_{A}=a_{B}=a$, and $D_{A}=D_{B}=D_{C}$, Eq. (1.12) and (1.13) are given by

$$
\begin{aligned}
& D_{a p p}=D_{c} \varepsilon \\
& q_{m}^{2}=\left(9 / R_{0}^{2}\right) \varepsilon
\end{aligned}
$$

with $R_{0}^{2}=N a^{2}$, the mean-squared end-to-end distance of polymer coils.

The linearlized theory is expected to be applicable only to the very early stage of SD because of the neglect of the nonlinear terms and only to systems where the contribution of the random thermal force is not significant.

The linearized theory predicts the following characteristics :

1) The exponential time-evolution of the order parameter (Eq. (1.6)) and hence the elastics scattered intensity $I(q, t)$,

$$
I(q, t) \equiv(\text { const. })\left\langle S_{q}(t)^{2}\right\rangle_{T}=I(q, 0) \exp [2 R(q) t]
$$

2) The mode of the fluctuations with a maximum growth rate has a constant wavenumber $q_{m}$ independent of time, or the scattering vector $q_{m}$ at which the scattered intensity becomes maximum is independent of time,

$$
q_{m}(t=0) \sim t^{0}
$$

where $q_{m}(t=0)$ is the value $q_{m}$ in the linear regime.

3) The $q$-dependent of the growth rate is given by Eq. (1.11) or $R(q) / q^{2}$ linearly decreases with increasing $q^{2}$.

4) $R(q)$ has a crossover from positive to negative at $q_{c}$, i.e., 


$$
\begin{aligned}
& R(q) / q^{2}=D_{\text {app }}\left[1-q^{2} / q_{c}^{2}\right] \\
& q_{c}^{2}=q_{m}^{2}
\end{aligned}
$$

5) $D_{a p p}$ and $q_{m}^{2}$ should be given by Eq. (1.18) and (1.19).

The linearized theory of SD should be regarded at best as an approximate theory applicable in the very early stage. Even in the early stage as time elapses, the intrinsically nonlinear nature of the time-evolution behavior of the order parameter becomes increasingly important, which is due to the increasing contribution of the nonlinear terms, e.g. $g S^{3}$ in the TDGL equation (Eq. (1.3)). Langer, Bar-on, and Miller [63] (LBM) solved the TDGL equation by using a "decoupling approximation" on the probability distribution functional of the order parameter. Their results predict (i) nonexponential growth of the order parameter and elastic scattered intensity and (ii) time-dependent growth of the fluctuation size $q_{m}^{-1}$. Thus, instead of Eq. (1.20) and (1.21), LBM predicts

$$
\begin{aligned}
& \tilde{I}\left(q_{m}, t\right) \sim t^{0.81} \\
& q_{m} \sim t^{-0.212}
\end{aligned}
$$

even in the very early stage where $\tilde{I}$ is reduced scattered intensity.

In the late stage of spinodal decomposition the scaling relations of $q_{m}(t)$ and $I_{m}(t) \equiv I\left(q_{m}, t\right):$

$$
\begin{aligned}
& q_{m}(t) \sim t^{-\alpha} \\
& I_{m}(t) \sim t^{\beta}
\end{aligned}
$$

have been traditionally investigated (both experimentally and theoretically) to probe the time-evolution of the structure pattern in the early-to-late stage of unmixing of small-molecule systems.

Kawasaki [57] and Kawasaki-Ohta [64] generalized the TDGL equation by introducing the hydrodynamic interaction in order to account for coarsening behavior in the early-to-late stage of the unmixing of small-molecule fluid mixtures. That is, the Fokker-Planck equation for the time evolution of the probability functional of the order parameter was written as

$$
\begin{aligned}
& \frac{\partial}{\partial} P(\{S\}, t)=\mathrm{L} P(\{S\}, t) \\
& \mathrm{L}=\mathrm{L}_{\mathrm{TDGL}}+\mathrm{L}_{\mathrm{HD}}
\end{aligned}
$$

where $\mathrm{L}_{\text {TDGL }}$ is the stochastic operator associated with the thermodynamic potential, 


$$
\mathrm{L}_{\mathrm{TDGL}}=-L \int d \boldsymbol{r} \frac{\delta}{\delta S(\boldsymbol{r})} \nabla^{2}\left[\frac{\delta}{\delta S(\boldsymbol{r})}+\frac{\delta \Phi}{\delta S(\boldsymbol{r})}\right]
$$

and $\mathrm{L}_{\mathrm{HD}}$ is the operator associated with the hydrodynamic interaction.

$$
\begin{gathered}
\mathrm{L}_{\mathrm{HD}}=2 \iint d \boldsymbol{r}_{1} d \boldsymbol{r}_{2} \frac{\delta}{\delta S\left(\boldsymbol{r}_{1}\right)} \nabla_{1} S\left(\boldsymbol{r}_{1}\right) \cdot T\left(\boldsymbol{r}_{1}-\boldsymbol{r}_{2}\right) \\
\nabla_{2} S\left(\boldsymbol{r}_{2}\right)\left[\frac{\delta}{\delta S\left(\boldsymbol{r}_{2}\right)}+\frac{\delta \Phi(t)}{\delta S\left(\boldsymbol{r}_{2}\right)}\right]
\end{gathered}
$$

$T(r)$ is the Oseen tensor given by

$$
T(r)=\frac{1}{8 \pi \eta}\left(\frac{1}{r} \delta_{\alpha \beta}+\frac{1}{r^{3}} r_{\alpha} r_{\beta}\right)
$$

Theory predicts there is no simple scaling relation, the power being a function of the time $t$. The theory can predict the limiting value of $\alpha$ obtained by LBM at the short time limit where the gradient of the order parameter $\nabla S$ is so small that the hydrodynamic interaction $\mathrm{L}_{\mathrm{HD}}$ is insignificant compared with the thermodynamic interaction $\mathrm{L}_{\text {TDGL }}$. On the other hand, at the long time limit, the term $\nabla S$ becomes significant so that the contribution of $\mathrm{L}_{\mathrm{HD}}$ much outweighs the contribution of $\mathrm{L}_{\text {TDGL }}$ and hence $\alpha$ approaches unity, as predicted by Siggia [65], although the Kawasaki approach cannot be rigorously applied to the very late stage since it is based on Langer's expansion.

Furukawa [66, 67] extended the Kawasaki-Ohta theory by developing the scaling idea. The theory can describe the behavior over a much wider range of $\tau$, the reduced time as defined Eq. (2.45) in later, applicable to the early-to late stage SD. His predictions again gives no simple scaling relation, $\alpha$ varying with $t$ or $\tau$ from 0.2 to 1 .

The time-evolution behavior of the unmixing structure during isothermal phase separation of the critical mixture is illustrated schematically in Figure 1.1. The figure shows the experimentally justified model for the spatial concentration profile of one constitution component $\phi_{A}(\boldsymbol{r}, t)$. In the very early stage of SD where the time-evolution behavior can be approximated by the linearized theory, the wavelength $\Lambda_{m}$ for the dominant mode of the fluctuation, which is equivalent to $\Lambda$ in Figure 1.1(a), is given by

$$
\Lambda_{m}=2 \pi / q_{m}(t=0)
$$

and is invariant with $t$. The amplitude of fluctuations $\Delta \phi$ exponentially increases 
(a) Early stage of SD

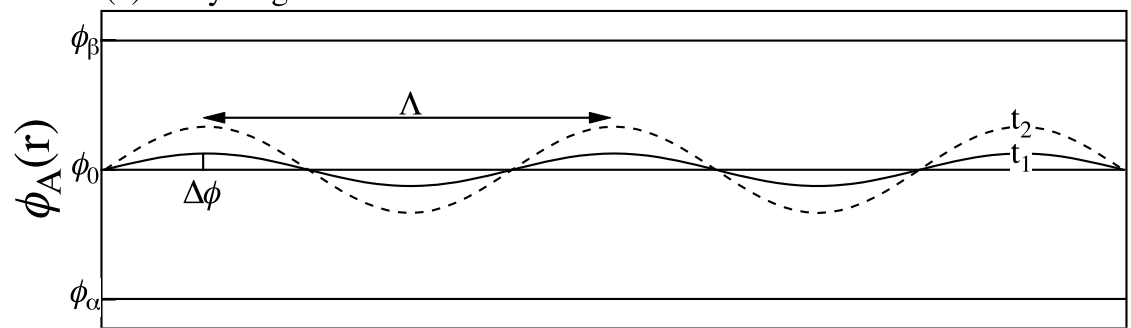

(b) Intermadiate stage of SD r

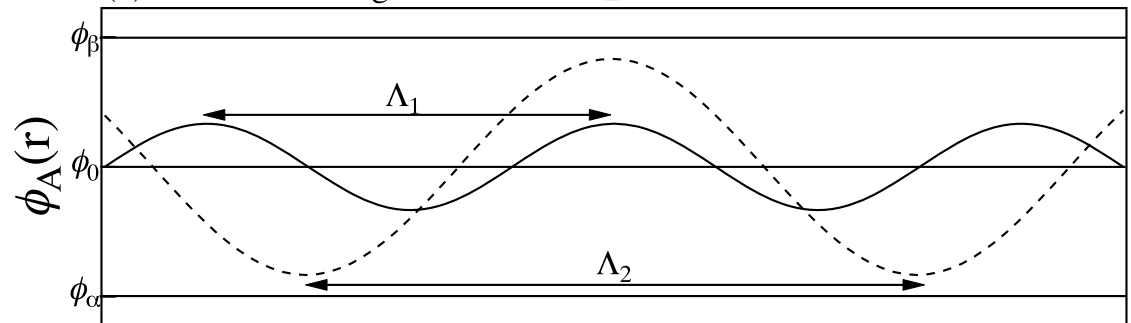

(c) Late stage of SD

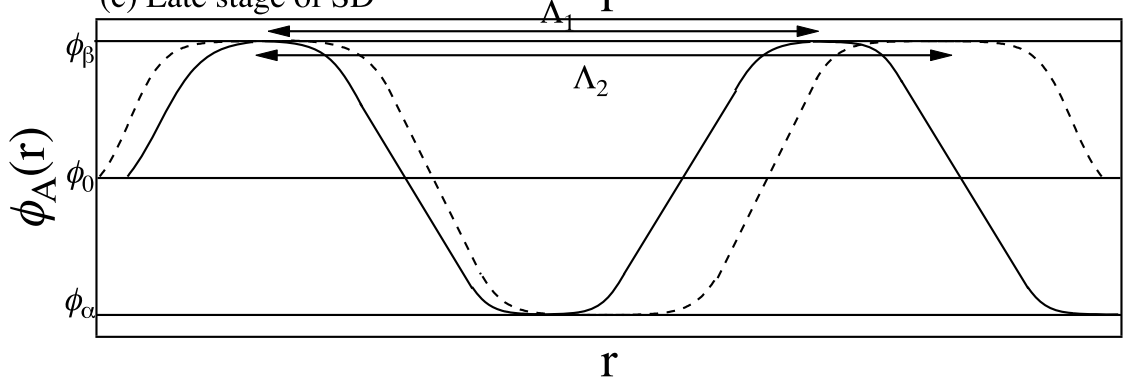

Figure 1.1: Schematic representation of spatial concentration fluctuation of one component in a critical mixture at three stage of unmixing: (a) early stage of SD, (b) intermediate stage of SD, and (c) late stage of SD. 
with $t\left(t_{1}<t_{2}\right)$. In the intermediate stage of SD (Figure 1.1(b)) where various coarsening processes may occur as described above and as will be described further, both the wavelength of the dominant mode of fluctuations and the amplitude of the fluctuations grow with time $\left(\Lambda_{1}<\Lambda_{2}\right)$. The unmixing structures at different times have the self-similarity,

$$
\phi_{A}\left(\frac{r}{\Lambda_{1}}, t_{1}\right)=\phi_{A}\left(\frac{r}{\Lambda_{2}}, t_{2}\right)
$$

where $\phi_{A}$ is the concentration of one component, e.g., component $A$ in the mixture of $A$ and $B$. In this regime, elastic scattering theory predicts a relation between the scaling exponents $\alpha$ and $\beta$ given by

$$
\beta=3 \alpha
$$

On the other hand, in the intermediate stage of SD, scattering theory predicts the following inequality between the two exponents,

$$
\beta>3 \alpha
$$

In the late stage of SD where Eq. (1.34) and (1.35) hold, Binder-Stauffer (BS) [68, 69] predicted

$$
\alpha=\left\{\begin{array}{l}
\frac{1}{3} \text { (fluid mixtures) } \\
\frac{1}{6} \text { (solid mixtures) (BS - process) }
\end{array}\right.
$$

based on the diffusion and coalescence of clusters. In this regime, the Lifshitz-Slyozov [70] model (or Ostward ripening mechanism) also predict a $1 / 3$ power for solid mixtures on the basis of the vaporization-condensation mechanism.

$$
\alpha=\frac{1}{3} \quad \text { (solid mixtures, LS - process) }
$$

Siggia [65] predicted $\alpha=1$ by taking into account the motion of the interface driven by the interfacial tension $\sigma$

$$
q_{m}(t) \sim(\eta / \sigma) t^{-1}
$$

where $\eta$ is the viscosity. Siggia's model seems to account for the limiting behavior of the coarsening with the greatest power $\alpha$ in the long-time and dissipative regime.

In the late stage of SD, Binder $[68,69]$ proposed the idea of dynamical scaling, in that the structure function $S(\boldsymbol{q}, t)$ or the scattering intensity profile $I(\boldsymbol{q}, t)$ of the unmixing system at time $t$ can be scaled with a single length parameter $\Lambda(t)$, 


$$
S(\boldsymbol{q}, t) \sim \Lambda(t)^{d} \tilde{S}[q \Lambda(t)]
$$

where $d$ is the dimensionality of the systems, $\boldsymbol{q}$ is the scattering vector

$$
|\boldsymbol{q}|=(4 \pi / \lambda) \sin (\theta / 2)
$$

$\lambda$ and $\theta$ are, respectively, the wavelength of $\mathrm{X}$-ray, neutron, or light in the medium and the scattering angle in the medium, and $\tilde{S}$ is a universal scaling function. Furukawa [71] predicted the universal scaling function

$$
\tilde{S}(x) \sim x^{2} /\left(\gamma / 2+x^{2+\gamma}\right)
$$

where

$$
x=q \Lambda(t)
$$

and $\gamma$ is defined as

$$
\gamma=\left\{\begin{array}{cc}
d+1 & \text { for the off }- \text { critical mixture (cluster regime) } \\
2 d & \text { for the critical mixture (percolation regime) }
\end{array}\right.
$$

\subsubsection{Nucleation and Growth (NG)}

The process of nucleation and growth is not peculiar to polymers, but observed in many materials, and we consider it briefly. The specific point making up the differences to the case of a spinodal decomposition is the existence of an activation barrier. The reason for its occurrence is easily recognized. Figure 1.2 shows the change of the Gibbs free energy, $\Delta G$, following from the formation of spherical precipitate of the new equilibrium phase. $\Delta G$ depends on the radius $r$ of the precipitate, as described by the equation

$$
\Delta G(r)=-\frac{4 \pi}{3} r^{3} \Delta g+4 \pi r^{2} \sigma
$$

with

$$
\Delta g=g\left(\phi_{0}\right)-g\left(\phi^{\prime \prime}\right)
$$

Eq. (1.44) emanates from the view that $\Delta G$ is set up by two contributions, one being related to the gain in the bulk Gibbs free energy of the precipitate, the other to the effect of the interface between particle and matrix. This interface is associated with an excess free energy, and the symbol $\sigma$ stands for the excess free energy per unit area.

Since the building up of the interface causes an increase in the free energy, a barrier $\Delta G_{\mathrm{b}}$ develops, which first has to be overcome before growth can set in. The passage over this barrier constitutes the nucleation step. Representing an activated process, it occurs with a rate given by the Arrhenius equation 


$$
v_{\text {nuc }} \sim \exp -\frac{\Delta G_{\mathrm{b}}}{k T}
$$

whereby $\Delta G_{\mathrm{b}}$ is the barrier height

$$
\Delta G_{\mathrm{b}}=\frac{16 \pi}{3} \frac{\sigma^{3}}{(\Delta g)^{2}}
$$

Eq. (1.47) follows from Eq. (1.44) when searching for the maximum. $\Delta G_{\mathrm{b}}$ increases with decreasing distance from the binodal, where we have $\Delta g=0$. The change is illustrated by the curves in Fig. 1.2 which were calculated for different values of the ratio $\Delta g / \sigma$. We learn from this behavior that, in order to achieve reasonable rates, nucleation requires a certain degree of supercooling (or overheating, if there is an upper miscibility gap).

Nucleation and growth occurs if the unmixing is induced at a temperature near to the binodal, where the system is still stable with regard to small fluctuations. Further away from the binodal this restricted 'metastability' gets lost and spinodal decomposition sets in.

\subsubsection{Dewetting}

There are two kinds of situations in wetting of a substrate by liquid as shown in Figure 1.3. For example, a water droplet spreads completely on a cleaned glass substrate. On the other hand, a water droplet on the plastics does not. This wetting and dewetting can be dominated by a spreading coefficient $(S)$, which is defined by the difference between surface energy of liquid on the dried surface $\left(E_{D}\right)$ and that on the wetting surface $\left(E_{\mathrm{W}}\right)$,

$$
\begin{aligned}
& S=E_{D}-E_{W}, \\
& S=\gamma_{S O}-\left(\gamma_{S L}+\gamma\right) .
\end{aligned}
$$

where $\gamma_{S O}, \gamma_{S L}$ and $\gamma$ are surface tensions are between solid and air, solid and liquid, liquid and air, respectively.

If $S$ is positive, liquid is stable. On the other hand, if $S$ is negative, dewetting occurs when thickness is less than the critical thickness. It is known that the thickness of critical dewetting $\left(e_{\mathrm{c}}\right)$ is given by

$$
e_{\mathrm{c}}=2 \kappa^{-1} \sin \frac{\theta_{\mathrm{E}}}{2}
$$




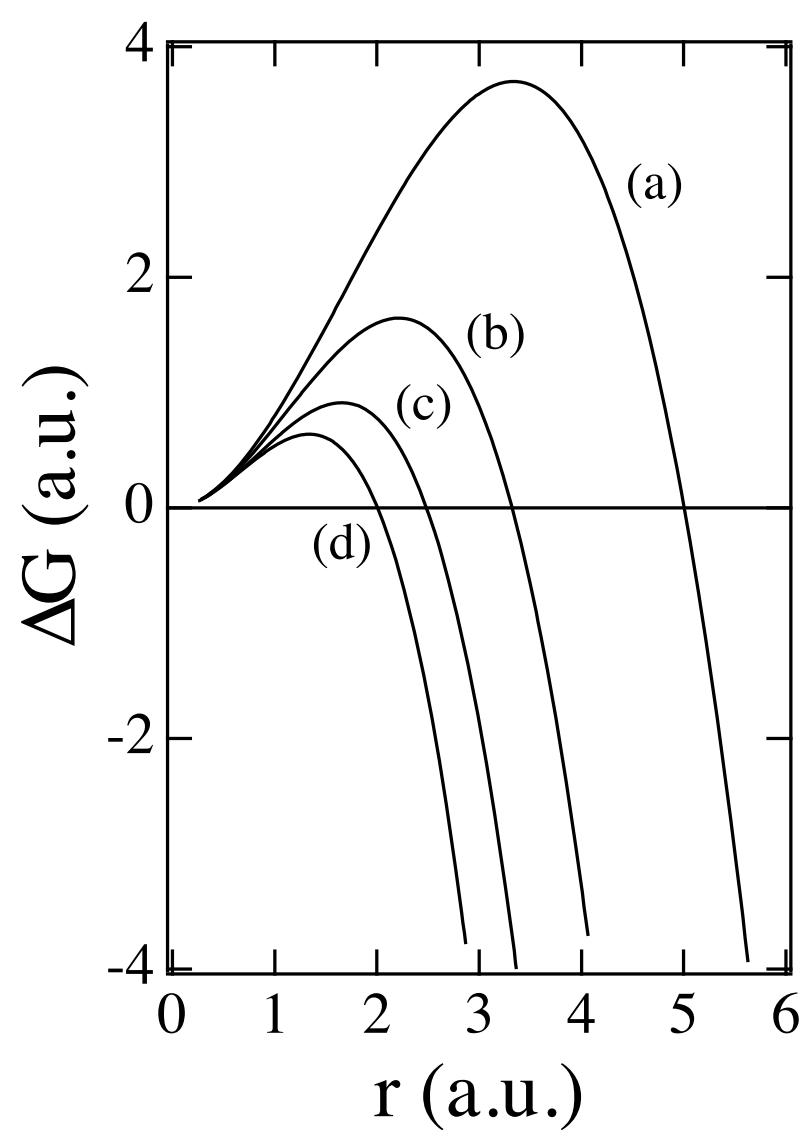

Figure 1.2: Activation barrier encounted during formation of a spherical nucleus. Curves (a) (d) correspond to a sequence 2:3:4:5 of values for $\Delta g / \sigma$. 

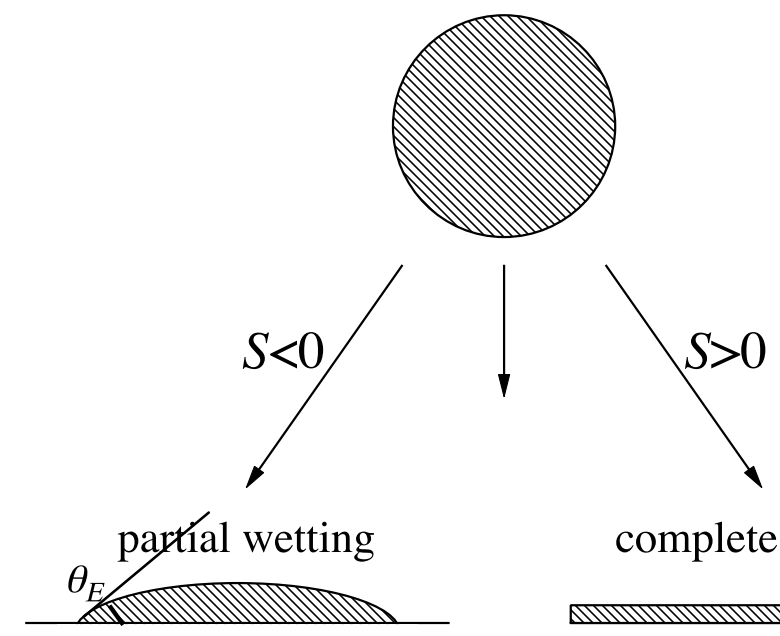

complete wetting

Figure 1.3: Two situations when puddle put on.

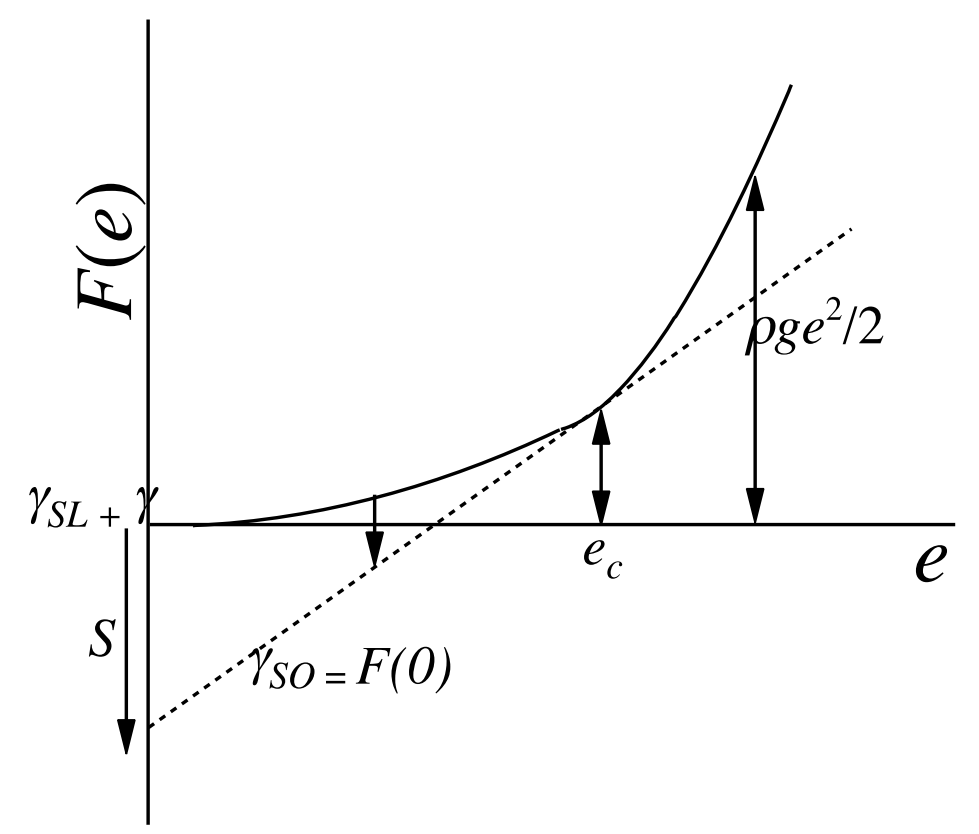

Figure 1.4: Schematic representation of free energy for dewetting liquid. 
where $\kappa^{-1}$ is capillary length which have millimeter order, $\theta_{\mathrm{E}}$ is contact angle between the liquid surface and he substrate (see Figure 1.3). In order to understand the critical thickness, it is necessary to consider the free energy of thin film $F(e)$ as thefunction of thickness (Fig. 1.4).

For thick films, $F(e)$ is given by the sum of surface energy and gravitational energy.

$$
F(e)=\gamma_{\mathrm{SL}}+\gamma+\frac{1}{2} \rho g e^{2}
$$

where $g$ is gravitational acceleration and $\rho$ is the density. When $S<0\left(\gamma_{\mathrm{SO}}<\gamma_{\mathrm{SL}}+\gamma\right)$, it is understood from the Maxwell construction law that equilibrium is hold between dry solid surface and a thin film with thickness of $e_{\mathrm{c}}$. Therefore $e_{\mathrm{c}}$ is the thickness of the puddle. When the thickness is $e<e_{\mathrm{c}}$, it is clear from this construction law that a thin film is always in metastable state, because the free energy is lowered when the phase separation occurs into two phases to coexist with each other. From Eq. (1.51), when surface tension of wetting solid $\left(\tilde{\gamma}_{\mathrm{SO}}\right)$ is defined as $\tilde{\gamma}_{\mathrm{SO}}=\frac{\partial(F(e) A)}{\partial A}$, it is given by

$$
\tilde{\gamma}_{\mathrm{SO}}=\gamma_{\mathrm{SL}}+\gamma-\frac{1}{2} \rho g e^{2}
$$

The equilibrium between a dry surface and the puddle can be written as

$$
\tilde{\gamma}_{\mathrm{SO}}=\gamma_{\mathrm{SO}}
$$

This expression is equal to an expression that shows local balance of on a part of the puddle, and its solution is give by $e=e_{\text {c }}$.

Equation (1.52) does not take into account the microscopic energy in thin film. Hence, $F(e \rightarrow 0)$ actually does not approach $F(0)=\gamma_{\text {so }}$. To improve this inconsistency it is necessary to consider the effect of the long distance force $(P(e))$ in $F(e)$. It is given by

$$
F(e)=\gamma_{\mathrm{SL}}+\gamma+P(e)+\frac{1}{2} \rho g e^{2} .
$$

Hereafter we consider a simple liquid in which $P(e)$ is dominated by the van der Waals force. Therefore, $P(e)$ is described as

$$
P(e)=\frac{A}{12 \pi e^{2}}
$$

It is noted that $A=A_{\mathrm{SL}}-A_{\mathrm{LL}}$ is the Hamaker constant for a liquid on a solid, and it is 


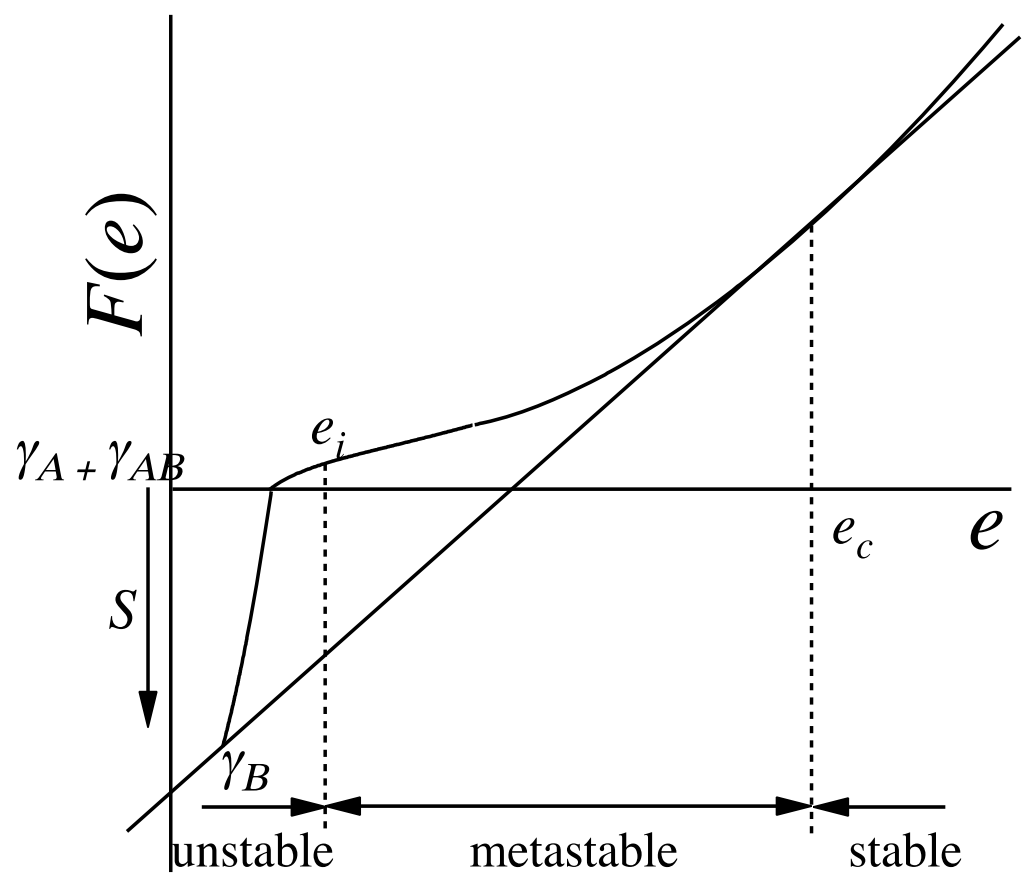

Figure 1.5: Schematic representation of two kinds of dewetting mechanism (spinodal decomposition and nucleation and growth). 
negative in this situation.

Figure 1.5 indicates the energy of the wetting solid $(F(e))$ in a wide range thickness from microscopic to macroscopic region. From this figure, it is shown that dewetting has two mechanisms similar to phase separation.

In the case of macroscopic thickness, the curvature of $F(e)$ is positive because gravity is predominant, and then thin film becomes metastable. Nucleation of dry region is necessary for the dewetting and grows when the diameter is larger than the critical value. This growth was theoretically [72] and experimentally [73, 74] studied. Nucleation and growth dewetting firstly studied by C. Redon [75].

In the case of microscopic thickness, the curvature of $F(e)$ is negative because the gravity energy is negligible compared with the long range force, and hence thin film in an unstable region. Therefore, the capillary wave is amplified and thin film spontaneously separate to various drops to form polygonal patterns. It is called spinodal decomposition similar to the case of phase transition. This was firstly discovered by G. Reiter [76].

\subsection{Current Situation of Polymer Blend Thin Film Studies}

Reich et al. first studied the thickness and composition dependence of cloud point $\left(T_{c}\right)$ for the phase separation systematically by laser light scattering and revealed that film thickness influences the phase separation behavior of PS/PVME blends when the film thickness is smaller than $1 \mu \mathrm{m}$, though this effect is substrate dependent [77]. Krausch et al. investigated the film thickness dependence of the spinodal wave for a binary polymeric blend and revealed that spinodal wave originating from the polymer-substrate interface was remarkable when the film thickness was smaller than $1 \mu \mathrm{m}$ [41]. Moreover, the offset of spinodal waves originating from two interfaces, the air-polymer and the polymer-substrate, was confirmed when the film thickness was smaller than $500 \mathrm{~nm}$. After his pioneering works, the various researchers studied the phase separation of polymer blend thin films. Tanaka et al. studied the surface structure of PS/PVME ultra-thin films using atomic force microscopy (AFM) and observed dewetted droplets in one phase region for films thinner than twice the radius of gyration. In this report, polymeric films whose thicknesses are less than twice the radius of gyration of an unperturbed chain, $2 R_{\mathrm{g}}$, can be defined as two-dimensional ultra-thin blend films [78]. In this thesis, we also similarly use this ultra-thin film by 
the same definition. Karim et al. [79, 80] also reported similar undulation of the free surface in the two phase region where dPS is encapsulated by PVME in the thin film. These observations suggest that phase separation and dewetting competitively occur in thin and ultra-thin films of polymer blend. Small-angle light scattering studies on the kinetic of phase separation of PS/PVME blend thin films [81] show that the phase separation proceeds by a spinodal decomposition mechanism in a thick film (= $180 \mathrm{~nm})$ while in the thin film (= $30 \mathrm{~nm})$ it switches to nucleation and growth.

For other polymer blend systems, Stamm et al. [82] have extensively investigated blend thin films of poly(p-methylstyrene) (PpMS) and deuterated polystyrene (dPS), which was a weakly incompatible system, to find that bilayer structure is formed via phase separation and the upper layer of poly(p-methylstyrene) dewetted on the lower layer of deuterated polystyrene. The blend thin films of poly (styrene-ran-acrylonitrile) (SAN) and deuterated poly (methyl methacrylate) (dPMMA) were also studied by some groups [44, 83-86]. Composto et al. recently examined the kinetics of phase separation in the blend thin films displaying discrete and bicontinuous domain morphologies [83], and found breakdown of dynamic scaling in the bicontinuous domain growth due to suppression of lateral hydrodynamic pumping with decreasing the film thickness while the scaling was hold in the discrete case. The same group also studied the dewetting mechanism of thin blend films of SAN and dPMMA in a wide thickness range [44, 84, 85] and presented a morphology map [86] based on the pattern development mechanism for thickness values and bulk composition between $50-1000 \mathrm{~nm}$ and $\phi_{\mathrm{PMMA}}=0.3-0.8$, respectively. As for the dewetting Composto et al. [84, 85] have proposed the capillary fluctuation mechanism for the blend thin films of SAN and dPMMA $\left(R_{\mathrm{g}}<d<150 R_{\mathrm{g}}, R_{\mathrm{g}}\right.$ being radius of gyration of a polymer). On the other hand, Chung et al. [87] have recently shown that the phase separation in thin films of SAN and dPMMA $(d=550 \mathrm{~nm})$ drove the dewetting and the capillary fluctuations failed to explain the dewetting. Liao et al. [88] have experimentally demonstrated that the composition fluctuation mechanism [54, 89 91] induced the dewetting in thermodynamically stable ultrathin blend films of SAN and dPMMA in one phase region. Thus, the dewetting mechanism in blend thin films is still a controversial problem.

Phase separation and dewetting of various polymer blend systems were well characterized by various methods, however the definite mechanism of phase separation 
and dewetting is still unknown. Even a quantitative evaluation of the film thickness dependence of the dewetting and the phase separation has not been investigated experimentally in PS/PVME. On the other hand, blend thin films of SAN and dPMMA is well done. As will be seen in this thesis, in order to understand the phase separation and dewetting mechanisms we will perform systematic studies on PS/PVME blend in a wide thickness range from hundred $\mu \mathrm{m}$ to few $\mathrm{nm}$.

\subsection{Outline of This Thesis}

The contents of this thesis are as follows;

In Chapter 2, the fundamental theories of scattering and reflectivity are described. In addition, we will introduce the instruments used in this study in detail.

In Chapter 3, the morphology and kinetics of phase separation as well as dewetting in PS/PVME blend thin films in a wide thickness $d$ range from semi-infinite thickness (65 $\mu \mathrm{m})$ to that close to radius of gyration $R_{\mathrm{g}}$ of PS and PVME chains $\left(d \sim 1.5 R_{\mathrm{g}}\right)$ are studied. From these results, we elucidate the relation between phase separation and dwetting in blend thin films of PS and PVME in the two phase region. We found that the dewetting occurred in a spinodal decomposition (SD) mechanism after a long incubation time in a thickness range below $200 \mathrm{~nm}$ although direct evidences for the layered structure were not obtained in this Chapter.

In Chapter 4, we investigated morphology and kinetics of dewetting in thin and ultrathin films of PS/PVME blends in a thickness $d$ range from thin film $\left(2.5 R_{\mathrm{g}}\right)$ to that less than radius of gyration $R_{\mathrm{g}}$ of PS and PVME chains ( $d \sim 0.5 R_{\mathrm{g}}$ ) to elucidate the relation between phase separation and dewetting of PS and PVME in the two phase region.

In Chapter 5, we have mainly studied structural development in the depth direction in the incubation period before the dewetting for PS/PVME thin films below 200 nm using neutron reflectivity to elucidate the dewetting mechanism.

In Chapter 6, structural development for PS/PVME thin films below 200 nm at various temperatures in the two phase region before and after the dewetting are studied using time-of-flight NR measurement. We discussed phase separation as well as dewetting in the depth direction using specular reflectivity and in the in-plane direction using off- specular reflectivity.

In Chapter 7, we studied the effects of the composition in the PS/PVME blend thin 
films on the dewetting morphology and kinetics below 200 nm using optical microscope, and structural development in the depth direction before the dewetting using neutron reflectivity.

In the end of the thesis, in summary, the whole results and discussions are summarized. 


\section{References}

[1] Jones R L, Richards R W. Polymers at Surface and Interfaces. Cambridge: Cambridge University Press; (1999).

[2] Karim A, Kumar S. Polymer Surfaces, Interfaces and Thin Films. Singapore: World Scientific; (2000).

[3] Bucknall D G. Progress in Materials Science 49, 713 (2004).

[4] Mueller-Buschbaum P, Bauer E, Wunnicke O, Stamm M. J. Phys.: Condens. Matter 17, S363 (2005)

[5] Keddie J L, Jones R A, Cory R A. Europhys. Lett. 27, 59 (1994).

[6] Keddie J L, Jones R A, Cory R A. Faraday Discuss. 98, 219 (1994).

[7] Forrest J A, Dalnoki-Veress K, Stevens J R, Dutcher J R. Phys. Rev. Lett. 77, 2002 (1996).

[8] Kawana S, Jones R A L. Phys. Rev. E, 63, 021501 (2001).

[9] Miyazaki T, Nishida K, Kanaya T. Phys. Rev. E, 69, 06183 (2004).

[10] Orts W J, Zanten J H v, Wu W, Satija S K. Phys. Rev. Lett. 71, 867 (1993).

[11] Fukao K, Miyamoto Y. Phys. Rev. E, 61, 1743 (2000).

[12] DeMaggio G B, Frieze W E, Gidley D W, Zhu M, Hristov H A, Yee A F. Phys. Rev. Lett. 78, 1524 (1997).

[13] Kanaya T, Miyazaki T, Watanabe H, Nishida K, Yamano H, Tasaki S, Bucknall D B, Polymer 44, 3769 (2003).

[14] Gau H, Herminghaus S, Lenz P, Lipowsky R, Science 283, 46 (1999).

[15] Boltau M, Walheim S, Mlynek J, Krausch G, Steiner U, Nature 391, 877 (1998).

[16] Higgins A M, Jones R A L, Nature 404, 476 (2000).

[17] Vrij A, Discuss. Faraday Soc. 42, 23 (1966).

[18] Brochard-Wyart F, Daillant J, Can. J. Phys. 68, 1084 (1990).

[19] Brochard-Wyart F, Martin P, Redon C, Langmuir 9, 3682 (1993).

[20] Reiter G, Phys. Rev. Lett. 68, 75 (1992).

[21] Xie R, Karim A, Douglas J F, Han C C, Weiss R A, Phys. Rev. Lett. 81, 1251 (1998).

[22] Bishof J, Sherer D, Herminghaus S, Leiderer P, Phys. Rev. Lett. 77, 1536 (1996).

[23] Seeman R, Herminghaus S, Jacobs K, J. Phys. Condens. Matter 13, 4925 (2001).

[24] Lambooy P, Phelan K C, Haugg O, Krausch G, Phys. Rev. Lett. 76, 1110 (1996). 
[25] Krausch G, J. Phys. Condens. Matter 9, 7741 (1997).

[26] Sferrazza M, Heppenstall-Butler M, Cubitt R, Bucknall D, Webster J, Jones R A L, Phys. Rev. Lett. 81, 5173 (1998).

[27] Flebbe T, Dunweg B, Binder K, J. Phys. II 6, 667 (1996).

[28] Binder K, Nielaba P, Pereyra V Z, J. Phys. B 104, 81 (1997).

[29] Binder K, Acta Polym. 46, 204 (1995)

[30] Jones R A L, Norton L J, Kramer E J, Bates F S, Wiltzius P, Phys. Rev. Lett. 66, 1326 (1991).

[31] Hashimoto T, in "Current Topics in Polymer Science Vol. II”, Ottenbrite R M, Utracki L A, Inoue S, Eds., Hanser, N.Y., pp.199 (1987).

[32] Nose T, Phase Transition 8, 245 (1987).

[33] Cahn J W, Hilliard J E, J. Chem. Phys. 28, 258 (1958).

[34] Furukawa H, Physica 123A, 497 (1984).

[35] Bruder F, Brenn R, Phys. Rev. Lett. 69, 624 (1992).

[36] Geoghegan M, Krausch G, Prog. Polym. Sci. 28, 261 (2002).

[37] Wang H, Douglas J F, Satija S K, Composto R J, Han C C, Phys. Rev. E 67, 061801 (2003).

[38] Nisato G, Ermi B D, Douglas J F, Karim A, Macromolecules 32, 2356 (1999).

[39] Puri S, Frisch H L, J. Phys. Condens. Matter 9, 2109 (1997).

[40] Plapp M, Gouyet J F, Phys. Rev. Lett. 78, 4970 (1997).

[41] Binder K, Puri S, Frisch H L, Faraday Discuss. 112, 103 (1999).

[42] Krausch G, Dai C, Kramer E J, Bates F K, Phys. Rev. Lett. 71, 3669 (1993).

[43] Krausch G, Kramer E J, Bates F K, Marko J F, Brown G, Chakrabarti A, Macromolecules 26, 5566 (1993).

[44] Sung L, Karim A, Douglas J F, Han C C, Phys. Rev. Lett. 76, 4368 (1996).

[45] Heier J, Kramer E J, Revesz P, Battistig G, Bates F S, Macromolecules 32, 3758 (1999).

[46] Wang H, Composto R J, Europhys. Lett. 50, 622 (2000).

[47] Haas C K, Torkelson J M, Phys. Rev. E 55, 3191 (1997).

[48] Genzer J, Kramer E J, Phys. Rev. Lett. 78, 4946 (1997).

[49] Kajiyama T, Tanaka K, Ohki I, Ge S, Yoon J, Takahara A, Macromolecules 27, 7923 (1994).

[50] Muller-Buschbaum P, Vanhoorne P, Scheumann V, Stamm M, Europhys. Lett. 40, 
655 (1997).

[51] Jandt K D, Heier J, Bates F S, Kramer E J, Langmuir 12, 3716 (1996).

[52] Hoppe H, Heuberger M, Klein J, Phys. Rev. Lett. 86, 4863 (2001).

[53] Clarke N, Eur. Phys. J. E 14, 207 (2004).

[54] Clarke N, Macromolecules, 38, 6775 (2005).

[55] Gunton J D, San Miguel M, Sahni P S, Phase Transitions and Critical Phenomena, vol. 8, eds. Academic Press, N.Y., pp.269 (1983).

[56] Cook H E, Acta Metall. 18, 297 (1970).

[57] Kawasaki K, Progr. Theor. Phys. 57, 826 (1977).

[58] Cahn J W, J. Chem. Phys. 42, 93 (1965).

[59] van Aartsen J, Eur. Polym. J. 6, 919 (1970).

[60] Pincus P, J. Chem. Phys. 75, 1996 (1981).

[61] Binder K, J. Chem. Phys. 79, 6387 (1983).

[62] Flory P J, Principle of Polymer Chemistry, Cornell Univ. Press, N.Y. (1953).

[63] Langer J S, Bar-on M, Miller H D, Phys. Rev. A11, 1417 (1975).

[64] Kawasaki K, Ohta T, Progr. Theor. Phys. 59, 362 (1978).

[65] Siggia E D, Phys. Rev. A20, 595 (1979).

[66] Furukawa H, Phys. Lett. Sect. A98, 28 (1983).

[67] Furukawa H, Adv. Phys. 34, 703 (1985).

[68] Binder K, Stauffer D, Phys. Rev. Lett. 33, 1006 (1974).

[69] Binder K, Phys. Rev. B15, 4425 (1977).

[70] Lifshitz I M, Slyozov V V, J. Phys. Chem. Solids. 19, 35 (1961).

[71] Furukawa H, Physica A. 123, 497 (1984).

[72] Taylor G I, Michael E, J. Fluid Mech. 58, 625 (1973).

[73] Paddy J F, Spec. Discuss. Faraday. Soc. 1, 64 (1971).

[74] Debregeas G, Brochard-Wyart F, J. Coll. Interface Sci. 190, 134 (1997).

[75] Redon C, Brochard-Wyart F, Phys. Rev. Lett. 66, 715 (1991).

[76] Reiter G, Phys. Rev. Lett. 68, 75 (1992).

[77] Reich S, Cohen Y, J. Polym. Sci.: Polym. Phys. Ed. 19, 1255 (1981).

[78] Tanaka K, Yoon J S, Takahara A, Kajiyama T, Macromolecules, 28, 934 (1995).

[79] Ermi B D, Karim A, Douglas J F, J. Poly. Sci., Part. B, 36, 191 (1998).

[80] Karim A, Slawecki T M, Kumar S K, Douglas J F, Satija S K, Han C C, Russell T P, Liu Y, Overnay R, Sokolov J, M. H. Rafailovich, Macromolecules, 857 (1998). 
[81] El-Mabrouk K, Belaiche M, Bousmina M, J. Coll. Interface Sci. 306, 354 (2007).

[82] Mueller-Buschbaum P, O'Neil S A, Affrossman S, Stamm M, Macromolecules, 31, 5003 (1998).

[83] Chung H, Composto R J, Phys. Rev. Lett. 92, 185704 (2004).

[84] Wang H, Composto R J, J. Chem. Phys. 113, 10386 (2000).

[85] Wang H, Composto R J, Interface Science, 11, 237 (2003).

[86] Chung H, Wang H, Composto R J, Macromolecules,39, 153 (2006).

[87] Chung H, Ohno K, Fukuda T, Composto R J, Macromolecules, 40, 384 (2007).

[88] Liao Y, Su Z, Sun Z, Shi T, An L, Macromol. Rapid Commun. 27, 351 (2006).

[89] Wensink K D F, Je'remoe B, Langmuir, 18, 413 (2002).

[90] Sharma A, Mittal J. Phys. Rev. Lett. 89, 1861 (2002).

[91] Sharma A, MIttal J, Verma R. Langmuir, 18, 10213 (2002). 


\section{Chapter 2}

\section{Basic Theories of Scattering and Reflectivity, and Experimental Techniques}

\subsection{Introduction}

Structure of polymer systems is generally investigated in both real and reciprocal spaces. The former studies are implemented using various kinds of microscopes such as electron microscope, atomic force microscope, and optical microscope. The latter is implemented by various kinds of scattering techniques such as wide-angle X-ray and neutron diffraction, small-angle X-ray and neutron scattering and light scattering. For the surface and interface studies, we also employ neutron reflectivity techniques. In this chapter we summarize the basic principles of scattering and reflectivity, and explain the scattering apparatus, the reflectivity apparatus and the microscopes used in this thesis in order to give a basis to understand the results presented.

\subsection{Theory of Scattering and Reflectivity}

In the following section, we describe the basic theories of scattering and reflectivity.

\subsubsection{Scattering Theory}

Depending on the system under study and the desired resolution, photons in the $\mathrm{X}$-ray and light range, or neutrons are used. The general set up of a scattering experiment is indicated schematically in Figure 2.1. There has an incident beam ofmonochromatic radiation with wavelength $\lambda$ and intensity $I_{0}$. It is scattered by a sample and the intensity $I$ of the scattered wave is registered by a detector at a distance $A$, under variation of the direction of observation. Employing the scattering vector $\boldsymbol{q}$, defined as

$$
\boldsymbol{q} \equiv \boldsymbol{k}_{f}-\boldsymbol{k}_{i}
$$

where $\boldsymbol{k}_{f}$ and $\boldsymbol{k}_{i}$ denote the wave vectors of the incident and the scattered plane waves, respectively. The result of a scattering experiment is usually expressed by giving the intensity distribution in $\boldsymbol{q}$-space, $I(\boldsymbol{q})$. In the majority of scattering experiments on polymers the radiation frequency remains practically unchanged 


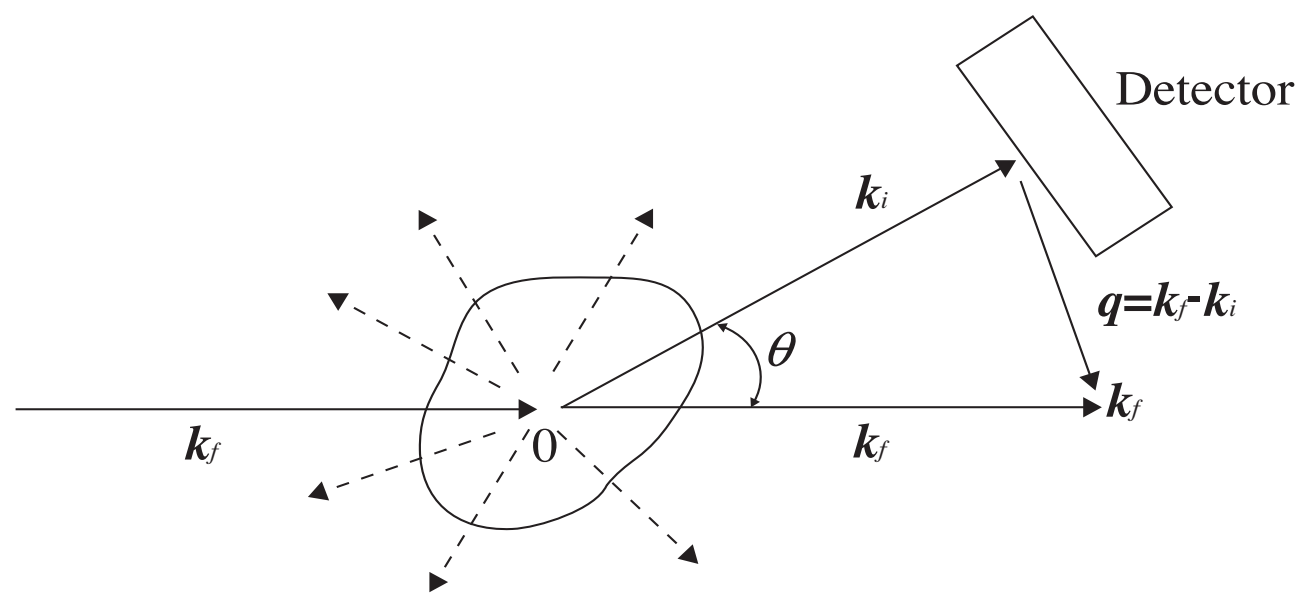

Figure 2.1: A schematic representation of the light-scattering experiment. 
because there is no energy transfer between the incident and scattered beam. They are so-called 'elastic scattering' while those in which energy transfer occurs are named 'inelastic scattering'. Then in the case of elastic scattering we have

$$
\left|\boldsymbol{k}_{f}\right|=\left|\boldsymbol{k}_{i}\right|=\frac{2 \pi}{\lambda}
$$

and $|\boldsymbol{q}|$ is related to the scattering angle $2 \theta$ by

$$
|\boldsymbol{q}|=\frac{4 \pi}{\lambda} \sin \theta
$$

\section{Principles for Static Scattering Experiments}

Two different functions can be used for representing scattering data in reduced forms. The first one is the differential scattering cross-section per unit volume of the sample described as

$$
\Sigma(\boldsymbol{q}) \equiv \frac{1}{V} \frac{d \sigma}{d \Omega}=\frac{1}{V} \frac{I(\boldsymbol{q}) A^{2}}{I_{0}}
$$

In light scattering experiments this function is called the Rayleigh ratio. While the effect of the volume is removed, $\Sigma(\boldsymbol{q})$ remains dependent on the scattering power of the particles in the sample, which varies with the applied radiation. For X-rays, the scattering power is related to the electron densities, for light scattering to the associated refractive indices and for neutron scattering to the scattering length densities. This dependence is eliminated in the second function which, however, can only be employed if the scattering can be treated as being due to just one class of particles. In polymer systems these can be identified with the monomeric units. For equal particles the scattering properties can be described by the scattering function $S(\boldsymbol{q})$, which is defined as

$$
S(\boldsymbol{q}) \equiv \frac{I(\boldsymbol{q})}{I_{m} N_{m}}
$$

where $N_{m}$ represents the total number of particles or monomers in the sample, and $I_{m}$ is the scattering intensity produced by one particle, if placed in the same incident beam. The scattering function $S(\boldsymbol{q})$ is often also addressed as the structure function or structure factor. The relationship between $\Sigma(\boldsymbol{q})$ and $S(\boldsymbol{q})$ is described by the equation as 


$$
\Sigma(\boldsymbol{q})=\left\langle c_{m}\right\rangle\left(\frac{\mathrm{d} \sigma}{\mathrm{d} \Omega}\right)_{m} S(\boldsymbol{q}),
$$

where $(\mathrm{d} \sigma / \mathrm{d} \Omega)_{m}$ represents the scattering cross section per particle or monomer and $\left\langle c_{m}\right\rangle$ stands for their mean density

$$
\left\langle\mathrm{c}_{\mathrm{m}}\right\rangle=\frac{N_{m}}{V} .
$$

Scattering diagrams generally emerge from the superposition and interference of the scattered waves emanating from all the particles in the sample. If we describe the amplitudes of single scattered waves at the point of registration by the detector in normalized form, the total scattering amplitude is obtained as

$$
C=\sum_{i=1}^{N_{m}} \exp \left(\mathrm{i} \varphi_{i}\right) .
$$

The phases $\varphi_{i}$ are simply determined by the particle positions $\boldsymbol{r}_{i}$ and the scattering vector $\boldsymbol{q}$ only, being given by

$$
\varphi_{i}=\boldsymbol{q} \boldsymbol{r}_{i} .
$$

Thus the scattering amplitude produced by a set of particles at locations $\boldsymbol{r}_{i}$ is formulated as

$$
C(\boldsymbol{q})=\sum_{i=1}^{N_{m}} \exp \left(\mathbf{i} \boldsymbol{q} \boldsymbol{r}_{i}\right) .
$$

The scattering intensity $I(\boldsymbol{q})$ is proportional to the square of $C$

$$
I(\boldsymbol{q}) \sim\left\langle|C(\boldsymbol{q})|^{2}\right\rangle .
$$

Since measurements require a certain time that ensemble average, expresses as brackets, are generally obtained. For ergodic systems the time average carried out by the detector equals the theoretical ensemble average. Gels are nonergodic systems as well as glasses so that the time average is not always equal to the ensemble average in generally used time scales in scattering measurements. The scattering pattern from gel using highly coherent laser has a granular pattern called speckle which reflects the fluctuations of discrete ensemble in the coherent region. As the normalization of the amplitudes of the single scattered waves is already implied in the definition of the scattering function Eq. (2.5), Eq. (2.11) can be completed to

$$
S(\boldsymbol{q})=\frac{1}{N_{m}}\left\langle|C(\boldsymbol{q})|^{2}\right\rangle .
$$


This is a basic equation of general validity and it may serve as starting point for the derivation of other forms of scattering equations. The calculation of the squared scattering amplitude is following.

The first formula is given directly by insertion of Eq. (2.10) to Eq. (2.12), leading to

$$
S(\mathbf{q})=\frac{1}{N_{m}} \sum_{i, j=1}^{N_{m}}\left\langle\exp \left[\mathbf{i q}\left(\mathbf{r}_{i}-\mathbf{r}_{j}\right)\right]\right\rangle \text {. }
$$

One can also use a continuum description and introduce the particle density distribution $c_{m}(\boldsymbol{r})$ instead of specifying the discrete positions $\boldsymbol{r}_{i}$ of all particles. First, the scattering amplitude for a single microstate, as represented by the associated density distribution

$$
C(\boldsymbol{q})=\int_{V} \exp (\mathrm{i} \boldsymbol{q} \boldsymbol{r}) \cdot\left[c_{m}(\boldsymbol{r})-\left\langle c_{m}\right\rangle\right] d^{3} \boldsymbol{r}
$$

Scattering occurs only when $c_{m}$ varies within the sample that we subtract the mean value $\left\langle c_{m}\right\rangle$. Thus it is obviously revealed that the scattering is directly relating to the fluctuations. We can also see that $C(\boldsymbol{q})$ equals the Fourier-transition of the fluctuations in the particle density. Insertion of Eq. (2.14) into Eq. (2.12) and carrying out the ensemble average yields

$$
S(\boldsymbol{q})=\frac{1}{N_{m}} \int_{v} \int_{v} \exp \left[\mathrm{i} \boldsymbol{q}\left(\boldsymbol{r}^{\prime}-\boldsymbol{r}^{\prime \prime}\right)\right]\left\langle\left[c_{m}\left(\boldsymbol{r}^{\prime}\right)-\left\langle c_{m}\right\rangle\right]\right\rangle \cdot\left[c_{m}\left(\boldsymbol{r}^{\prime \prime}\right)-\left\langle c_{m}\right\rangle\right] d^{3} \boldsymbol{r}^{\prime} d^{3} \boldsymbol{r}^{\prime \prime}
$$

For all macroscopically homogeneous systems, where the equation of

$$
\left\langle c_{m}\left(\boldsymbol{r}^{\prime}\right) c_{m}\left(\boldsymbol{r}^{\prime \prime}\right)\right\rangle=\left\langle c_{m}\left(\boldsymbol{r}^{\prime}-\boldsymbol{r}^{\prime \prime}\right) c_{m}(0)\right\rangle
$$

is approved. This equation can be reduced to a single integral. Substitution of $\boldsymbol{r}^{\prime}-\boldsymbol{r}^{\prime \prime}$ by $\boldsymbol{r}$ yields

$$
\left.S(\boldsymbol{q})=\frac{1}{\left\langle c_{m}\right\rangle} \int_{V} \exp (\mathrm{iqr}) \cdot\left[\left\langle c_{m}(\boldsymbol{r}) c_{m}(0)\right\rangle-\left\langle c_{m}\right\rangle^{2}\right]\right]^{3} \boldsymbol{r}
$$

This equation expresses $S(\boldsymbol{q})$ as the Fourier-transform of the space dependent correlation function of the particle density.

A third form of the basic scattering equation is obtained if structures are characterized with the aid of the pair distribution function $g(\boldsymbol{r})$. From the definition, the product $g(\boldsymbol{r}) d^{3} \boldsymbol{r}$ gives the probability that starting from a given particles, the particle itself or some other particle is found in the volume element $d^{3} \boldsymbol{r}$ at a distance $\boldsymbol{r}$. The pair distribution function $g(\boldsymbol{r})$ is composed of a sum of two contributions which are self-contributions and the contributions of the other particles. For fluid 
systems with short-range order, the limiting value of the pair distribution function $g(\boldsymbol{r})$ at large distance $(r \rightarrow \infty)$ equals the mean density $\left\langle c_{m}\right\rangle$. As follows directly from the definitions, density distribution function and pair distribution function are related by

$$
\left\langle c_{m}(\boldsymbol{r}) c_{m}(0)\right\rangle=\left\langle c_{m}\right\rangle \cdot g(\boldsymbol{r}) .
$$

Insertion of the equation in Eq. (2.17) gives

$$
S(\boldsymbol{q})=\int_{V} \exp (\mathrm{i} \boldsymbol{q} \boldsymbol{r}) \cdot\left[g(\boldsymbol{r})-\left\langle c_{m}\right\rangle\right] d^{3} \boldsymbol{r} .
$$

It is once again revealed that the scattering function equals a Fourier-transform, now of the pair distribution function. At the same time, we follow the asymptotic values of $S$ for large values of $\boldsymbol{q}$ from this equation. In the limit $\boldsymbol{q} \rightarrow \infty$ only the contribution of the self-correlation part is left, and we find $S(\boldsymbol{q} \rightarrow \infty) \rightarrow 1$. It can be concluded that there are neither constructive nor destructive interferences between the particles for large $\boldsymbol{q}$ so that they behave like incoherent scatterers.

In the case of isotropic system, both vector parameters $\boldsymbol{q}$ and $\boldsymbol{r}$ can be treated as length parameters $q(=|\boldsymbol{q}|)$ and $r(=|\boldsymbol{r}|)$, respectively. Then the Fourier-relation between $g(r)$ and $S(q)$ has the form as

$$
S(\boldsymbol{q})=\int_{r=0}^{\infty} \frac{\sin q r}{q r} 4 \pi r^{2}\left[g(r)-\left\langle c_{m}\right)\right] d r
$$

In this chapter, it was formulated three equivalent relations, Eq. (2.13), (2.17) and (2.19), which may be employed in the evaluation of scattering data. All three equations express a Fourier-relation between $S(\boldsymbol{q})$ and functions which describe properties of the microscopic structure in statistical terms.

\subsubsection{Reflectivity Theory}

Much of the simple theory of reflection is presented in optics textbooks; a classic and clear presentation is to be found in Born and Wolf [1]. The general theory of the reflection of waves, both electromagnetic and particle, is dealt with in more detail in the book by Lekner [2]. Reviews of x-ray and neutron reflectivity, including their application to polymers, are available [3, 4]. In this section we concentrate on neutron reflectivity, primarily because of the advantage offered by deuterium labeling, though as x-ray reflectivity can also provide useful information, contrasts between reflection of neutrons and $\mathrm{x}$-rays will be pointed out where necessary.

We consider a plane wave traveling in medium 0 incident on the smooth surface of 
medium 1 (Figure 2.2); the associated wave-vectors in each medium are $k_{0}$ and $k_{1}$ and the refractive index at the boundary is given by $n=k_{1} / k_{0}$.

$$
n=1-\lambda^{2} A+\mathrm{i} \lambda C,
$$

where the complex term accounts for any absorption in medium 1 . For neutron beams

$$
\begin{gathered}
A=N b /(2 \pi), \\
C=n \sigma_{A} /(4 \pi),
\end{gathered}
$$

Where $N$ is the atomic number density of medium 1 and $b$ the bound atom coherent scattering length. The term $\sigma_{A}$ is the absorption cross section of the atomic species making up medium 1. Values of $b$ and $\sigma_{A}$ vary in an unpredictable manner across the periodic table and tabulated values are available [5]. For the atoms occurring in most polymers, absorption cross sections are either zero or negligible and the absorption term in equation (2.21) can generally be ignored. For polymeric species and solvents of low relative molecular mass $\mathrm{Nb}$ can be replaced by the scattering length density of the polymer segment of solvent molecule, $\rho$, and

$$
\rho=\frac{N_{A} d \sum_{i} b_{i}}{m},
$$

where $d$ is the physical density of the polymer (or solvent), $m$ is the relative molecular mass of the segment or solvent molecule and $\sum_{i} b_{i}$ is the sum of the bound atom coherent scattering lengths of the atoms making up the unit considered. For x-rays a similar expression is obtained:

$$
\rho=\frac{N_{A} d}{m} \sum_{i} z_{i} r_{\rho} .
$$

Here $r_{\rho}$ is the electron radius $\left(2.82 \times 10^{-13} \mathrm{~cm}\right)$ and $\sum_{i} z_{i}$ is the sum of the electrons in the species. The absorption coefficient, $C$, for x-rays is $\mu /(4 \pi)$, where $\mu$ is the mass absorption coefficient for the unit [6]. $\quad N_{\mathrm{A}}$ is Avogadro's number in these formulae.

At the interface between the two media the grazing angle of incidence, $\theta_{0}$, is related to the angle of refraction, $\theta_{1}$, by

$$
n_{0} \cos \theta_{0}=n_{1} \cos \theta_{1}
$$

If medium 0 is air, then $n_{0}$ is 1 and

$$
\cos \theta_{0}=n_{1} \cos \theta_{1}
$$


When $n_{1}$ is less than 1 there will exist a critical angle above which the angle of refraction will be real for all incident angles. (Note that, apart from light water and compounds that are heavily hydrogenous, most materials encountered in polymer science have a positive $A$ and thus $n_{1}<1$ ). At this critical angle of incidence, $\theta_{c}, \theta_{1}$ is zero and

$$
\cos \theta_{c}=n_{1} .
$$

When the critical angle is small, as is generally the case for neutron reflectivity, the cosine term can be expanded to give

$$
\theta_{c}=\left(\frac{\lambda^{2}}{\pi} \rho\right)^{1 / 2} .
$$

For a smooth polymer surface, the component of the incident beam's wave-vector normal to the surface, $k_{z 0}$, is $(2 \pi / \lambda) \sin \theta_{0}$, while in the polymer the $z$ component of the wave-vector, $k_{z 1}$, is $\left(k_{z 0}^{2}-4 \pi \rho\right)^{1 / 2}$, which can also be written $\left(k_{z 0}^{2}-k_{c}^{2}\right)^{1 / 2}$, where $k_{c}$ is the value of the component of the wave-vector normal to the surface at the critical angle. The reflectance of the interface between media 0 and 1 is given by the Fresnel formula

$$
r_{01}=\frac{k_{z 0}-k_{z 1}}{k_{z 0}+k_{z 1}} .
$$

The reflectivity $R$ is $r_{01} r_{01}^{*}$, with $r_{01}^{*}$ the complex conjugate of $r_{01}$; hence for the smooth interface the Fresnel reflectivity is given by

$$
R_{\mathrm{F}}(Q)=\left(\frac{Q-\left(Q^{2}-Q_{c}^{2}\right)^{1 / 2}}{Q+\left(Q^{2}-Q_{c}^{2}\right)^{1 / 2}}\right)^{2} .
$$

Hence we have used, instead of wave-vectors, the momentum transfer normal to the surface $Q=(4 \pi / \lambda) \sin \theta$, a notation preferred by those familiar with small-angle scattering. Replacing for $Q_{c}\left(=4 \pi^{1 / 2} \rho^{1 / 2}\right)$ we find that when $Q>>Q_{c}$ we can write

$$
R_{\mathrm{F}}(Q)=16 \pi^{2} \rho^{2} / Q^{4} \text {; }
$$

hence, for a smooth, sharp surface at sufficiently high $Q$ values, $Q^{4} R_{\mathrm{F}}(Q)$ should become a constant value determined only by the scattering length density of the bulk material.

When a single layer is interposed between the air and the bulk phase (i.e. the bulk material becomes a substrate) as in Figure 2.3 there is now reflection at each interface and a transmission coefficient for beam transport through the intervening layer. The reflected beam from the interface between media 1 and 2 can undergo reflection as well 


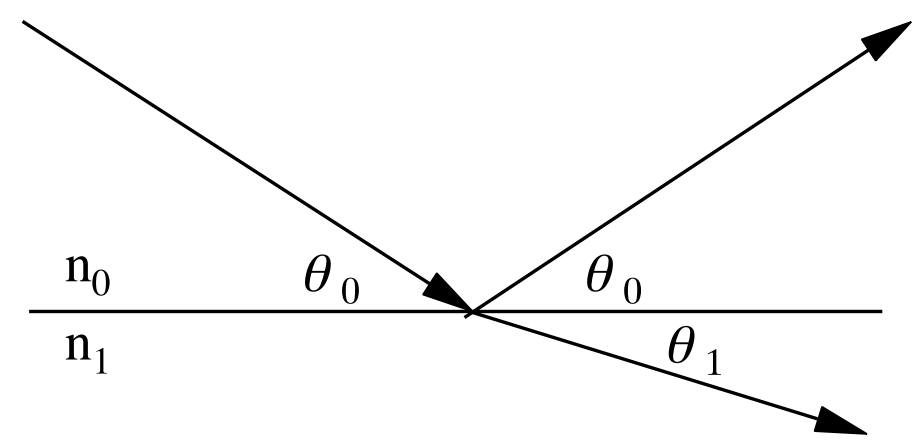

Figure 2.2: A schematic diagram of incident, reflected and refracted beams at the junction between two phases of refractive indices $n_{0}$ and $n_{1}$. In this case $n_{1}$ is smaller than $n_{0}$, as is commonly the case for neutrons incident on a material from air, and $\theta_{1}<\theta_{0}$.

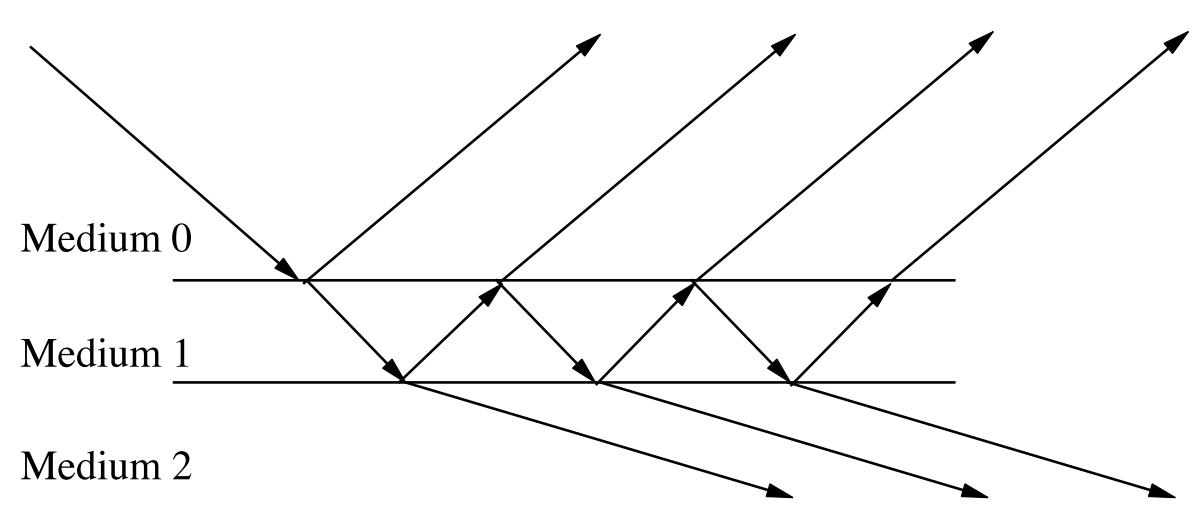

Figure 2.3: Multiple reflection and transmission for a beam incident on a layer of refractive index differing from that of the substrate. 
as refraction at the interface on going from medium 1 to medium 0 . This process continues until the transmission losses attenuate the beam to effective extinction. In this circumstance the reflectivity is given by

$$
R_{\mathrm{F}}=\left|\frac{r_{01}+r_{12} \exp (2 \mathrm{i} \beta)}{1+r_{01} r_{12} \exp (2 \mathrm{i} \beta)}\right|^{2}
$$

where the $r_{i j}$ teems the Fresnel reflectances calculated for each interface and $\beta$ is the phase shift or optical path length in medium 1:

$$
\beta=\frac{2 \pi}{\lambda} n_{1} d \sin \theta_{1}
$$

The externally reflected beams have different path lengths to the detector and interference between the reflected beams leads to maxima and minima being observed in the reflectivity. The separation of the minima in $Q, \Delta Q$, is related to the layer thickness by

$$
\Delta Q=2 \pi / d
$$

Similar formulae in terms of the Fresnel reflectances may be built up for situations with a small number of discrete layers. A larger number of layers demands the use of a more general method that is easily coded for numerical computation. The optical matrix method used in the reflectivity of light from stratified media [1] is often used. These methods are particularly relevant to polymer surfaces and interfaces for which the interfacial composition variation normal to the interface may change smoothly between limiting values as illustrated in Figure 2.4. In such cases the region over which the composition changes can be broken up into a series of discrete strips with a scattering length density approximating to that of the smooth decay. An additional factor is the occurrence of any roughness at the interfaces between such hypothetical strips (or indeed real roughness at the surface). Roughness at any interface reduces the specular reflectivity and is distinguishable in this respect from diffuseness of the interface. Its effect can be incorporated as a Gaussian smoothing factor on the Fresnel reflectivity coefficients. Such interfacial roughness can be incorporated into the optical matrix method if the method due to the Abeles is used in place of that due to Born and Wolf.

For this approach each layer in the interface as shown in Figure 2.4 is represented by a characteristic matrix; thus for layer $m$

$$
\mathbf{M}_{m}=\left\lfloor\begin{array}{cc}
\exp \left(\mathrm{i} \beta_{m-1}\right) & r_{m} \exp \left(\mathrm{i} \beta_{m-1}\right) \\
r_{m} \exp \left(-\mathrm{i} \beta_{m-1}\right) & \exp \left(-\mathrm{i} \beta_{m-1}\right)
\end{array}\right\rfloor,
$$




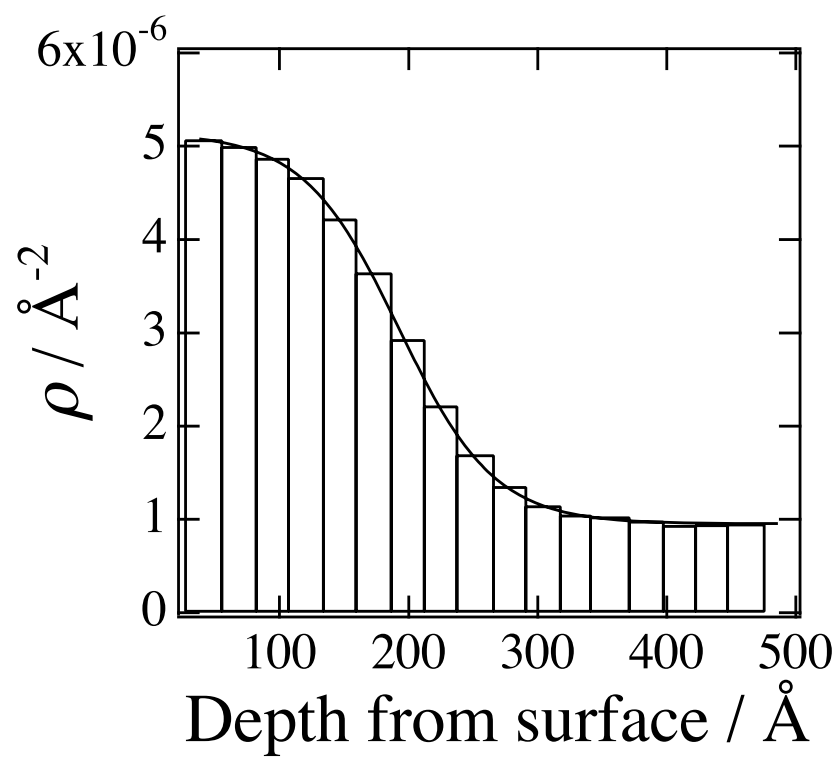

Figure 2.4: The gradual change in scattering length density (continuous line) and its approximation as a series of discrete layers.

\begin{tabular}{ccc}
\hline \hline Species & Unit chemical formula & $\rho\left(10^{-6} \AA^{-2}\right)$ \\
\hline H Polystyrene & $\mathrm{C}_{8} \mathrm{H}_{8}$ & 1.4 \\
D Polystyrene & $\mathrm{C}_{8} \mathrm{D}_{8}$ & 6.92 \\
H Polymethyl methacrylate & $\mathrm{C}_{5} \mathrm{H}_{8} \mathrm{O}_{2}$ & 0.90 \\
D Polymethyl methacrylate & $\mathrm{C}_{5} \mathrm{D}_{8} \mathrm{O}_{2}$ & 6.02 \\
H Polybutadiene & $\mathrm{C}_{4} \mathrm{H}_{6}$ & 0.45 \\
D Polybutadiene & $\mathrm{C}_{4} \mathrm{D}_{6}$ & 7.1 \\
H Polyethylene oxide & $\mathrm{C}_{2} \mathrm{H}_{4} \mathrm{O}$ & 0.56 \\
D Polyethylene oxide & $\mathrm{C}_{2} \mathrm{D}_{4} \mathrm{O}$ & 6.33 \\
Water & $\mathrm{H}_{2} \mathrm{O}$ & -0.56 \\
Heavy Water & $\mathrm{D}_{2} \mathrm{O}$ & 6.38 \\
H Cyclohexane & $\mathrm{C}_{6} \mathrm{H}_{12}$ & -0.28 \\
D Cyclohexane & $\mathrm{C}_{6} \mathrm{D}_{12}$ & 6.68 \\
\hline \hline
\end{tabular}

Table 2.1: Scattering length densities for selected polymers and solvents. 
where $\beta$ is the optical path length of layer $m$ as defined before and $r_{m}$ is the product of the Fresnel reflectance of the $m$ th interface $r_{m}^{\mathrm{f}}$ and a damping term that accounts for the roughness:

$$
\begin{aligned}
\mathbf{M}_{n} & =\mathbf{M}_{1} \mathbf{M}_{2} \mathbf{M}_{3} \cdots \mathbf{M}_{n} \\
& =\left\lfloor\begin{array}{ll}
\mathbf{M}_{11} & \mathbf{M}_{21} \\
\mathbf{M}_{12} & \mathbf{M}_{22}
\end{array}\right\rfloor .
\end{aligned}
$$

The reflectivity of the whole multilayer is then obtained as

$$
R(Q)=\frac{M_{21} M_{21}^{*}}{M_{11} M_{11}^{*}}
$$

This scheme lends itself well to computational calculation and, in principle, any number of layers can be incorporated, speed of processing being the limiting factor in simulating the reflectivity.

In particular terms, to observe an effect on the reflectivity due to some particular chemical species, there must be contrast between it and its surroundings. This is easily achieved using neutron beams because the bound atom coherent scattering length for deuterium is considerably different from that of hydrogen. Consequently, the scattering length density of the deuterated species is markedly different from that of the hydrogenous isomers (Table 2.1 shows some selected values of $\rho$ ). More detailed model and equation are refereed to a book [7].

\subsection{Experimental Apparatus}

In this work, we have used various kinds of instruments, including small-angle light scattering (SALS) apparatus [8], neutron reflectometer (NR), optical microscope (OM), atomic force microscope (AFM) and scanning near-field optical microscopy (SNOM).

\subsubsection{Small-Angle Light Scattering (SALS)}

During the phase separation process, especially the spinodal decomposition, characteristic power laws concerning the space and time appears. Therefore, the observation in the wide $q$ and intensity ranges should be essential to the studies of spinodal decomposition by the SALS instrument. The present SALS instrument has been constructed aiming to study the phase separation behavior for an extremely thin film. In the following sections, we describe the details of the SALS instrument to show the applicability of the instrument to thin films. 


\subsubsection{Instrumental Setup}

The optical geometry of the SALS instrument of the present study is schematized in Figure 2.5. The light source is a $50 \mathrm{~mW}$ He-Ne laser with the wavelength $\lambda=0.6328$ $\mathrm{mm}$, a product of Showa Optronics Co., Ltd. The beam power is reduced by the neutral density (ND) filters to meet each experimental requirement. Optical components, such as filters and lenses, are products of SIGMA KOKI Co., Ltd., if not otherwise specified. As was mentioned in the introduction section, a laser source is assumed to supply a beam of parallel light rays in theory, but in practice a small amount of unwished stray light is emitted from the laser-tube window (LW). If a scattering power of the sample is sufficient and/or a DPLS measurement is the subject of study, such a small amount of stray light does not matter so much. However, it will affect the signal-to noise ratio badly when a scattering power of the sample is faint. To eliminate the stray light, a pair of achromatic lenses $\left(L_{1}\right.$ and $\left.L_{2}\right)$ and an aperture (A) are introduced. In addition to the filtering purpose, the distances of $L_{1}$ to $A(=200 \mathrm{~mm})$ and $A$ to $L_{2}(=100 \mathrm{~mm})$ are arranged so as to reduce the beam diameter, since a small hole $(\phi=2.2 \mathrm{~mm})$ in a temperature-controlled stage for samples will become another origin of the stray light if the incident beam interferes with it.

Then the incident beam is focused on the detector plane (D) by a lens $L_{3}$. The place of the sample (S) is selectable between $L_{3}$ and $D$, unless the temperature-controlled stage mechanically interferes with $\mathrm{L}_{3}$ or $\mathrm{D}$. Thus the camera length (CL) can be flexibly selected to meet the experimental requirement. The longest and the shortest CLs of the present instrument are $400 \mathrm{~mm}$ and $100 \mathrm{~mm}$, respectively. The incident beam is finally screened by a beam stopper (BS) placed just before D. Since A and D are arranged optically conjugated, the stray light that is projected on D is limited within the shade of A in theory. However, in practice the diameter of the BS would better be selected depending on the status of use. In the present instrument a BS with $1.5 \mathrm{~mm}$ in diameter usually gives reasonable data up to the edge of the BS (see section 3.1). In this case, the lowest accessible $q$ is $0.021 \mu^{-1}$ for the longest CL (= $400 \mathrm{~mm})$ as will be shown later. When a sample has a very weak scattering power, a BS with $4.0 \mathrm{~mm}$ in diameter is used. This diameter is rather on the safe side. In this case, the lowest accessible $q$ is $0.056 \mu \mathrm{m}^{-1}$ for the longest CL.

If the monitoring of the transmittance of the sample is necessary, especially in the time-developing measurements, the center part of the BS is replaced by 


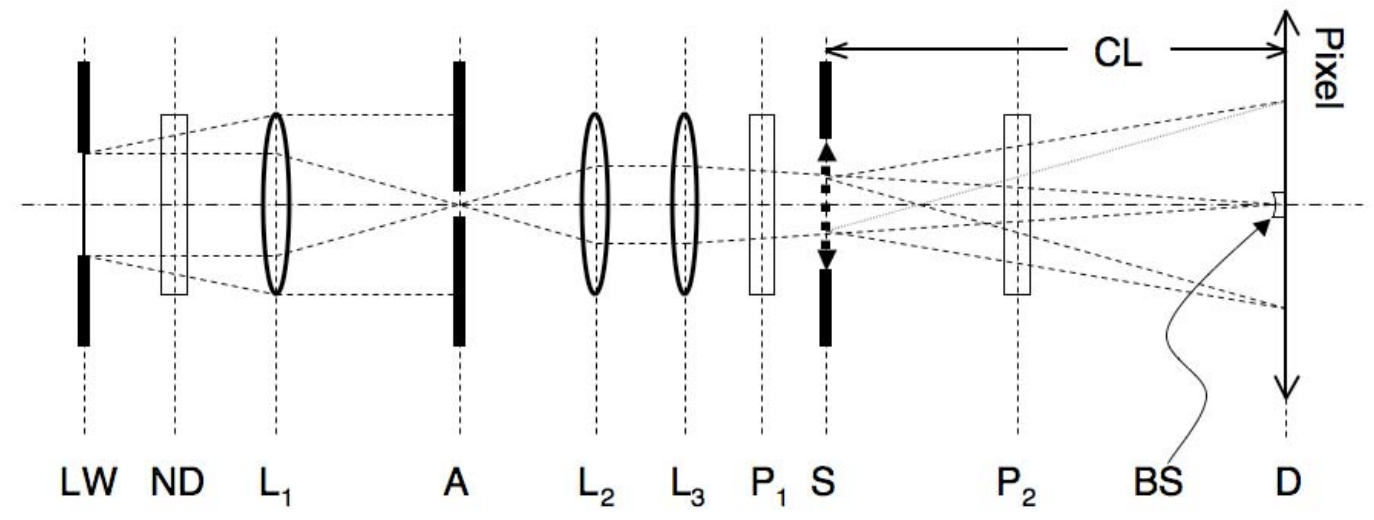

Figure 2.5: Schematic diagram of the optical geometry of the SALS instrument. LW: laser-tube window, ND: neutral density filters, $\mathrm{L}_{1}$ : lens $\left(\mathrm{F}_{1}=200 \mathrm{~mm}\right), \mathrm{A}$ : aperture $(\phi=0.3 \mathrm{~mm}), \mathrm{L}_{2}$ : lens $\left(\mathrm{F}_{2}=100 \mathrm{~mm}\right), \mathrm{L}_{3}$ : lens $\left(\mathrm{F}_{3}=450 \mathrm{~mm}\right), \mathrm{P}_{1}$ : polarizer, $\mathrm{S}$ : sample, $\mathrm{P}_{2}$ : analyzer, $\mathrm{BS}$ : beam stopper (semi-transparent), $\mathrm{D}$ : detector plane, and CL: camera length. 
semi-transparent material (exposed negative film). The intensity of the transmitted light is monitored by the pixels of the sensor just behind the center part of the BS. Thus the measurements of the scattering and the transmittance can be conducted simultaneously. Optionally, a polarizer $\left(\mathrm{P}_{1}\right)$ and an analyzer $\left(\mathrm{P}_{2}\right)$ are inserted before and after the specimen, respectively, for the DPLS measurements.

A CMOS image sensor, a product of Rad-icon Imaging Corp., is used for the detector in this study. The CMOS image sensor consists of $2048 \times 1024$ pixels and the each pixel covers $48 \mu \mathrm{m} \times 48 \mu \mathrm{m}$ area, accordingly ca. $10 \mathrm{~cm} \times 5 \mathrm{~cm}$ area is totally covered. The reported nominal dynamic range of the sensor is 12 bit $(=4096)$. The time necessary to save one scattering image using the provided software and a PC with a $2.8 \mathrm{GHz}$ Pentium 4 processor was $0.5 \mathrm{sec}$ without binning the pixels. This is the minimal time slice in the time-developing measurements of the present SALS instrument. This value will be reduced to $0.37 \mathrm{sec}$ in nominal if a PC with higher specification is used.

\subsubsection{Basic Performances}

\section{Beam Profile}

Figure 2.6(a) shows a two-dimensional beam profile on the detector plane (D) without a beam stopper. The profiles in the vicinity of the beam center are enlarged (see the scale). Figure 2.6(b) is a one-dimensional beam profile along the equator line that passing through the most intense point. The solid curve represents a Gaussian distribution fitted to the profile.

As seen from Figure 2.6(b), the full width at half maximum is ca. 8 pixels. This pixel value corresponds to $384(=8 \times 48) \mu \mathrm{m}$ in real space and also corresponds to $0.0048 \mu \mathrm{m}^{-1}$ in the absolute value of scattering vector $q(=4 \pi \sin \theta / \lambda$, where $\theta$ and $\lambda$ being the scattering angle and wave length, respectively) when the longest camera length $(C L=400 \mathrm{~mm}$ ) is selected. Namely, this $q$ value corresponds to the full limits of the $q$ resolution of the present instrument. The practical $q$ resolution will be discussed later. The base width, where the intensity is comparable to the level of a base line is ca. 30 pixels. This pixel value corresponds to $1.44 \mathrm{~mm}$. Therefore, the BS with $1.5 \mathrm{~mm}(>1.44 \mathrm{~mm})$ in diameter, as was mentioned in the former section, is considered a reasonable choice. 

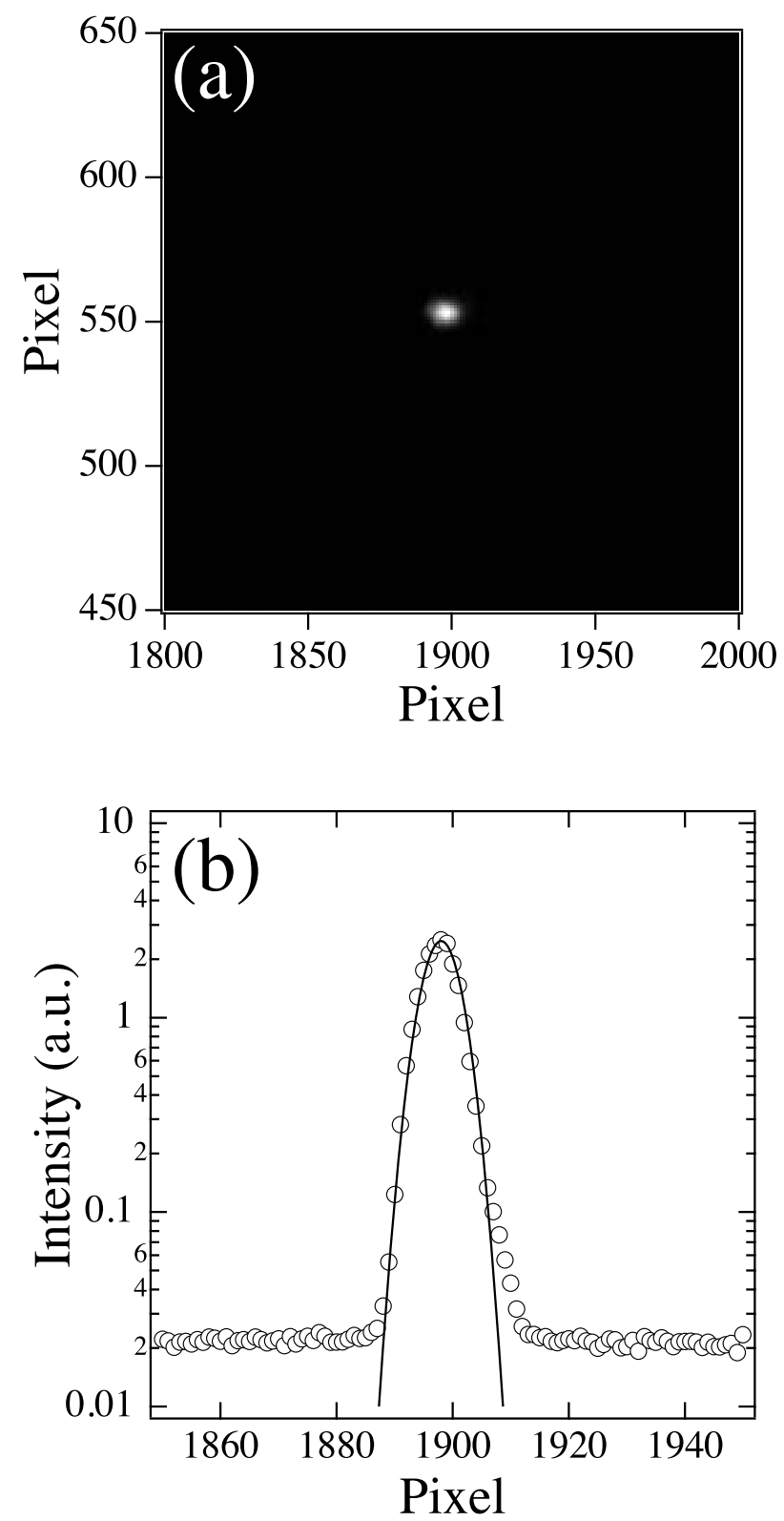

Figure 2.6: Two-dimensional (a) and a one-dimensional (b) beam profiles on the detector plane without a beam. 


\section{Dynamic Range and Linearity of Detector}

Although the nominal dynamic range of the sensor itself is 12 bit, the effective dynamic range that can be used for the data analysis depends on the status of use. Therefore, we have estimated a practical dynamic range of the present SALS instrument using the diffraction from a pinhole. The Fraunhofer diffraction from a pinhole, the so-called Airy pattern, is mathematically described as follows [9]:

$$
I(x)=\left[2 J_{1}(x) / x\right]^{2}
$$

where, $J_{2}(x)$ is the Bessel function of the first kind and $x$ is a product of $q$ and the radius of the pinhole $R(x=q R)$. Since the envelope curve for the Airy function shows the -3 power of $q$, a pinhole is often used as a standard sample to test SALS instruments [10].

Figure 2.7 shows a two-dimensional scattering image from a pinhole with $50 \mathrm{~mm}$ in diameter. Concentric periodic rings are clearly observed. A bright innermost ring adjacent to the shadow of the beam stopper is the 0 -th diffraction peak centered on $q=0$. The first and the higher order diffraction peaks from the $50 \mathrm{~mm}$ pinhole are perfectly resolved.

In order to evaluate the diffraction profile quantitatively, the two-dimensional scattering image is circularly averaged and converted into the one-dimensional intensity profile. Figure 2.8(a) shows a one-dimensional intensity profile taken at a single attenuation level. The cubic cosine correction for incident angle was conducted and the electronic noise, which was measured without beam, was subtracted, but the correction for the sensitivity of the image sensor was not conducted. The attenuation level is intentionally selected so as to saturate the counting capacity of the sensor. Accordingly, the intense part, i.e. the low $q$ region, of the intensity profile is plateaued at $4 \times 10^{3}$ in the given scale. On the other hand, the intensity in the highest $q$ region is ca. $2 \times 10^{0}$. In the meantime, the envelope curve for the measured diffraction peaks reproduces the -3 power of $q$ fairly well. Therefore, the practical dynamic range at a single attenuation level is evaluated as ca. $2 \times 10^{3}$ at least. The overall linearity of the sensor is also fairly well over three digits without the correction for the sensitivity, judging also from the reproduction of the power law of the envelope curve.

To meet a need for the wider dynamic range, multiple data sets taken at different attenuation levels are usually combined. Figure 2.8(b) shows such an example, where two data sets taken at two different attenuation levels are combined by overlapping the 


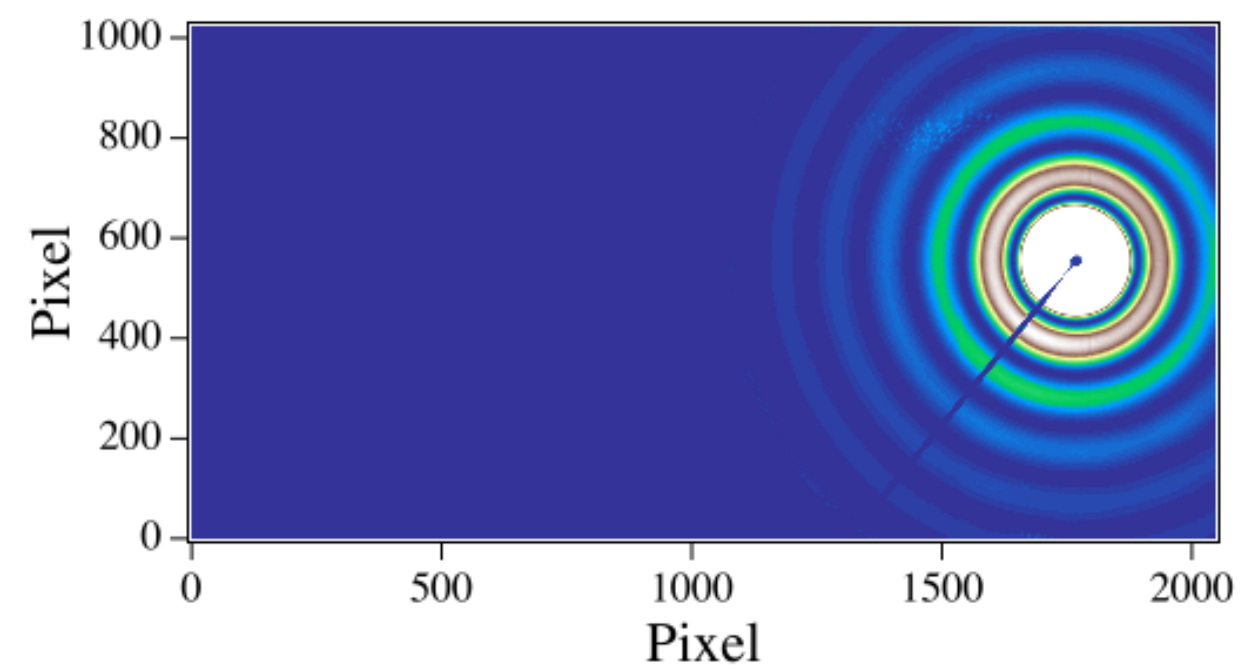

Figure 2.7: Two-dimensional scattering image from a pinhole with $50 \mu \mathrm{m}$ in diameter when CL is $400 \mathrm{~mm}$. 

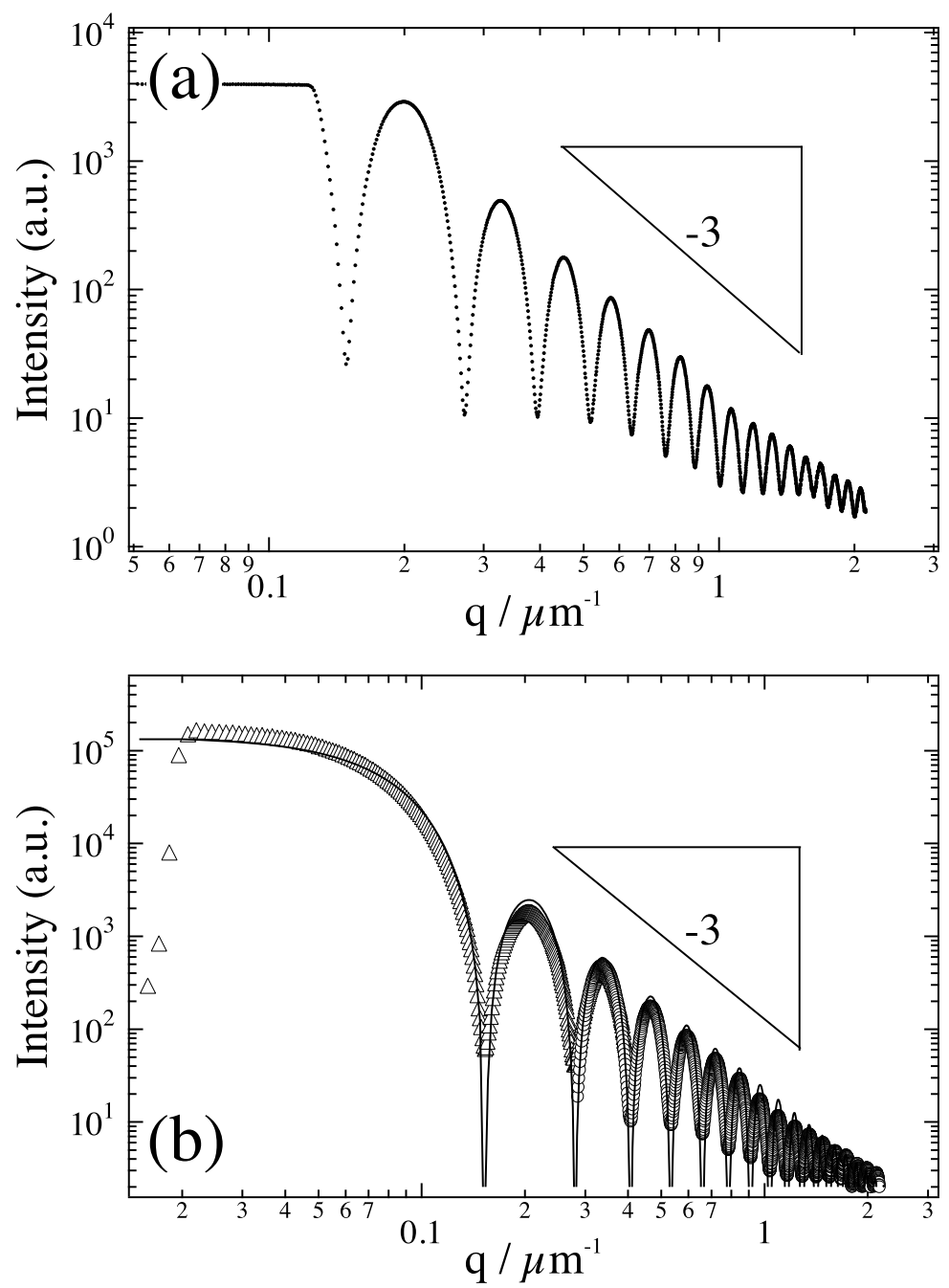

Figure 2.8: (a): One-dimensional intensity profile of the scattering from a pinhole with $50 \mu \mathrm{m}$ in diameter taken at a single attenuation level, and (b): combined profile using two data sets taken at two different attenuation levels. The solid curve represents the Airy function. 
2-nd diffraction peak. The relative attenuation factor for the data set of the higher intensity part was 1/60. The combined diffraction profile well reproduces the Airy function over five digits, except for the narrow minimums, which are somewhat smeared due to the resolution. In addition to the wide dynamic rage, a very wide scattering vector range from $q=0.02 \mu \mathrm{m}^{-1}$ to $2.2 \mu \mathrm{m}^{-1}$, i.e. three whole digits, is covered with a single camera length $(\mathrm{CL}=400 \mathrm{~mm})$. The higher limit of the scattering vector range is expandable to ca. $q=8 \mu \mathrm{m}^{-1}$ by using the shortest camera length (CL = $100 \mathrm{~mm}$ ). Namely, the diffraction from a Bragg spacing down to $0.8 \mu \mathrm{m}$ can be resolved.

\section{Resolution of Scattering Vector}

In order to verify the practical $q$ resolution, diffractions from pinholes with rather "macroscopic" sizes were measured. Figure 2.9 (a) and (b) show the one-dimensional intensity profiles from handmade pinholes with $0.53 \mathrm{~mm}$ and $1.00 \mathrm{~mm}$ in diameter, respectively. The diffraction peaks from the pinhole with $0.53 \mathrm{~mm}$ in diameter are perfectly resolved. Moreover, the diffraction peaks from the pinhole with $1.00 \mathrm{~mm}$ in diameter are still resolved. Thus the practical $q$ resolution is guaranteed less than $0.0063(=2 \pi / 1000) \mu \mathrm{m}^{-1}$. Namely, the full limits of the $q$ resolution $\left(0.0048 \mu \mathrm{m}^{-1}\right)$, which was estimated by the beam width, is almost attained.

\subsubsection{Neutron Reflectometers}

(1) MINE-II Reflectometer [11]

A cold neutron reflectometer (C3-1-2) is installed at a cold neutron guide tube of JRR-3M reactor of JAERI as shown in Figure 2.10. The monochromator system consists of two Ni-Ti multilayer mirrors with bilayer spacings of $110 \AA$ and two Ni-Ti multilayer mirrors with $120 \AA$, which are arranged in a partial polygon as shown in Figure 2.10. These multilayer mirrors have reflectivities of about 90\%. Neutrons from the guide tube are monochromatized by four sequential Bragg reflections and deflected by 23 degrees. The incident neutrons have a long wavelength of $8.8 \AA$ with wavelength resolution of $3.2 \%$. An angular resolution of 0.022 degrees can be achieved for a typical condition of $1 \mathrm{~mm}$ for slit 1 and slit 2 . With this resolution a

typical intensity of 1000 counts/sec is available for reflectivity measurements with a 
beam cross-section of $1 \mathrm{~mm} \times 36 \mathrm{~mm}$. The maximum size is $3 \times 40 \mathrm{~mm}^{2}$ at the exit of
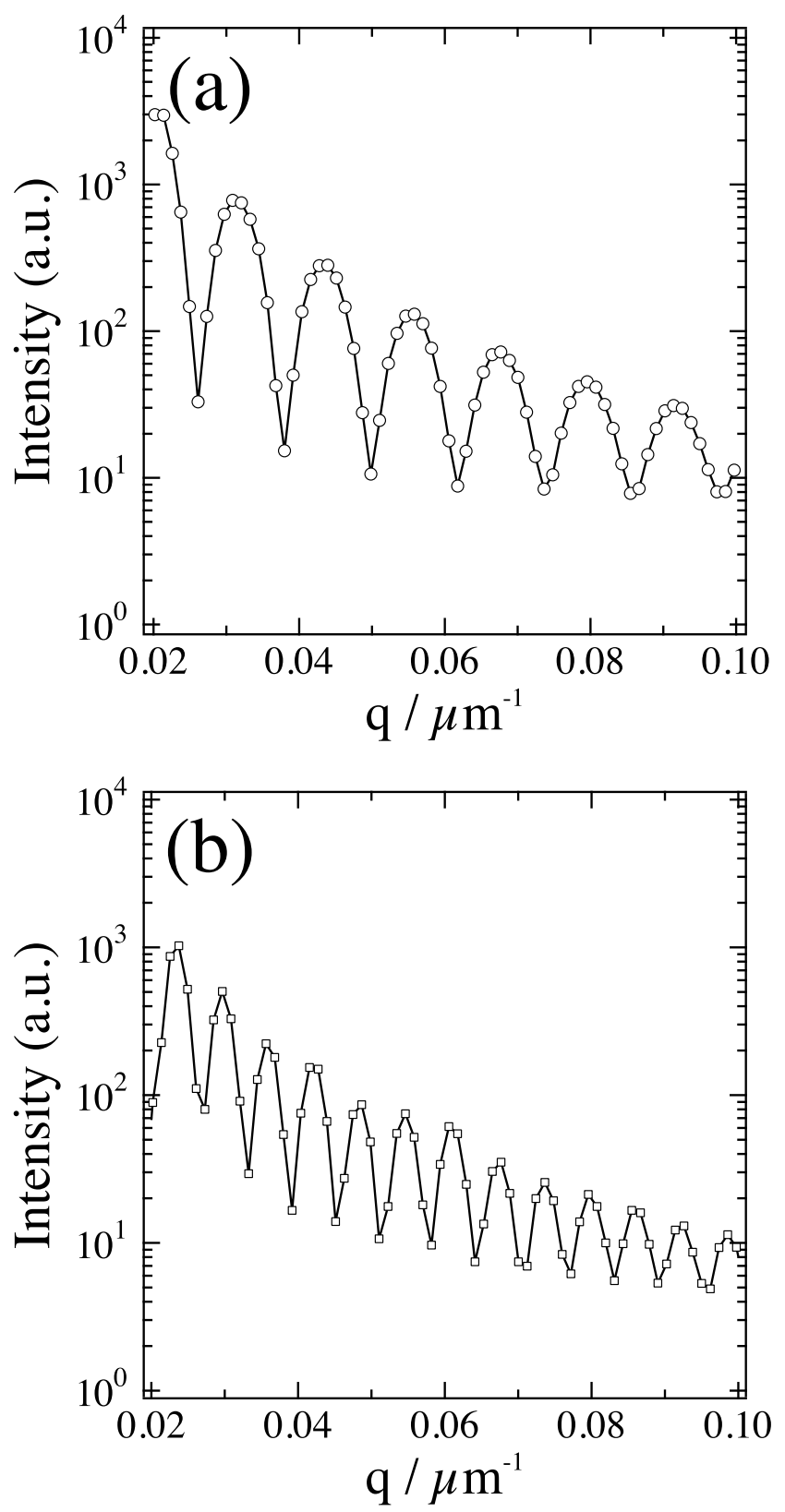

Figure 2.9: (a): One-dimensional intensity profile of the scattering from a pinhole with $0.53 \mathrm{~mm}$, and (b): with 1.00 $\mathrm{mm}$ in diameter. 
the monochromator system. A reflectivity curve is obtained within several hours and reflectivity of $10^{-5}$ can be measured, depending on the neutron background level.

The reflectometer is mounted in vertical geometry to yield a beam cross section of 3 $\times 40 \mathrm{~mm}^{2}$ and applicable to measurements of a mirror system which is formed on a flat substrate. The measurements are made automatically and 21 axes stepping motor drives are controlled by two personal computers.

The neutron detector system consists of a position-sensitive detector for measurements of off- specular profile and a He-3 detector for $\theta-2 \theta$ scans.

The neutron beam is monitored by neutrons transmitting through the third mirror of the monochromator system.

\section{(2) SPEAR Reflectometer}

Figure 2.11 indicates the schematic view of SPEAR located on Flight Path 9 in the Lujan Neutron Scattering Center at the Los Alamos National Laboratory. SPEAR is a time-of-flight (TOF) neutron reflectometer that measures chemical density profiles of thin layers (5 $\AA$ - $3000 \AA$ ) in a variety of different environments. The instrument uses an unpolarized neutron beam to study solid/solid, solid/liquid, solid/gas, and liquid/gas interfaces. SPEAR occupies flight-path 9 (FP9) and views a $20 \mathrm{~K}$ partially coupled liquid hydrogen $\left(\mathrm{LH}_{2}\right)$ moderator which provides a neutron flux of $9.4-106 \mathrm{n} / \mathrm{cm}^{2} / \mathrm{s}$ at a proton current of 100 microAmperes.

SPEAR's moderated neutrons are coarsely collimated into a beam with an average angle of $0.9^{\circ}$ to the horizontal that converges along the vertical direction at the sample position, which is $8.73 \mathrm{~m}$ from the moderator. The neutrons can be further collimated to the desired divergence by finely slitting the beam by a set of two slit packages. The largest vertical divergence is $+/-0.25^{\circ}$. In the horizontal direction, the beam converges at the detector at $12.40 \mathrm{~m}$ from the moderator with a maximum angular divergence of $0.45^{\circ}$.

Two choppers are used to define a typical neutron wavelength range of $1 \AA$ to $16 \AA$. The T0 chopper, which interrupts the beam during the initial flash of high-energy neutrons and gamma rays, significantly reduces the background that may limit reflectivity measurements. The frame-overlap chopper and mirrors define the wavelength band to be used and suppress frame-overlap background problems. With a polychromatic beam, a range of momentum transfer vectors $\left(Q_{z}\right)$ can be measured 


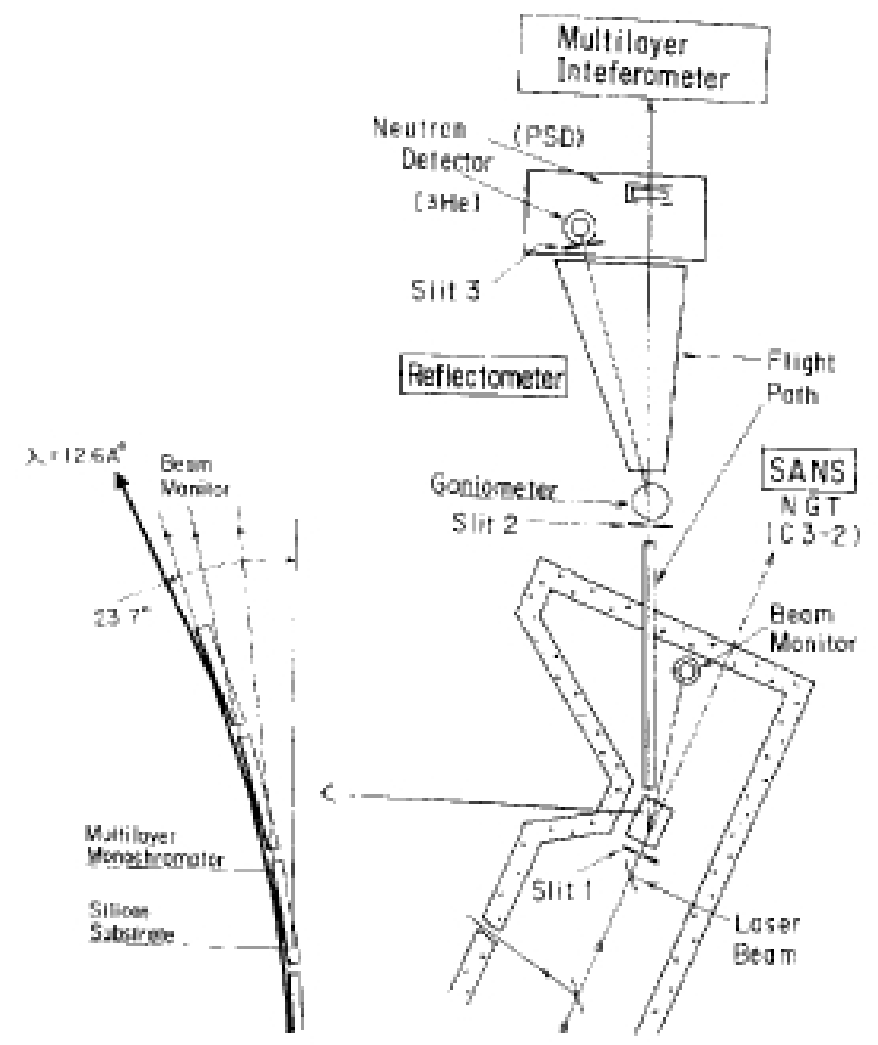

Figure 2.10: Schematic view of MINE.

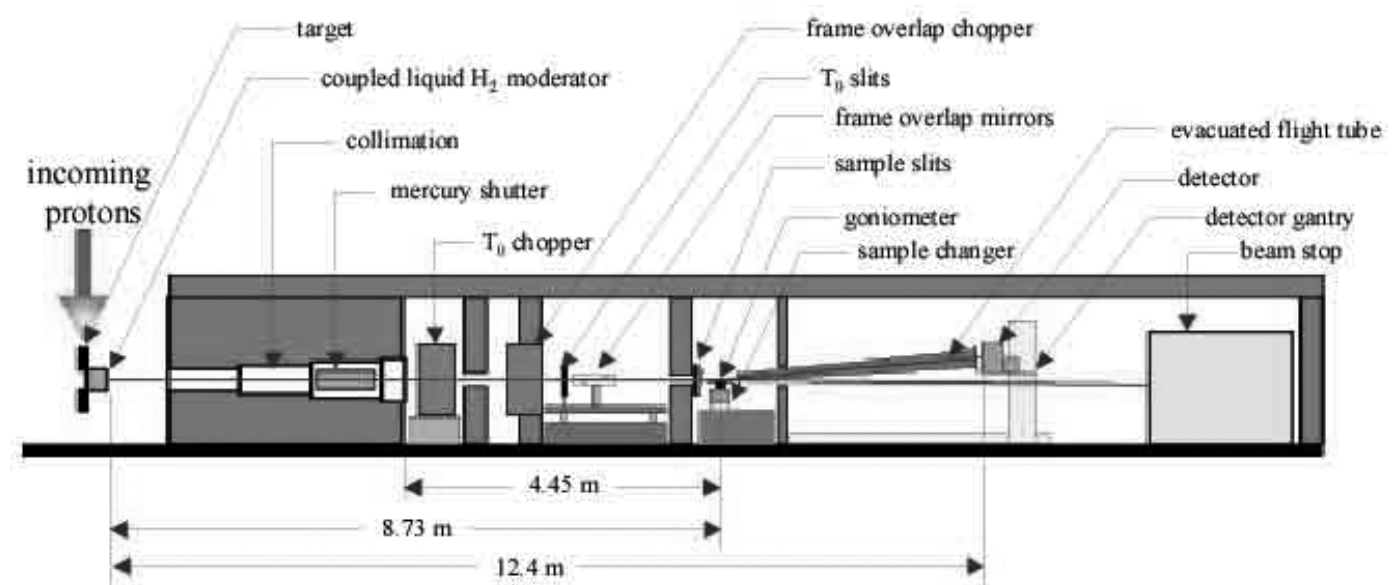

Figure 2.11: Schematic view of SPEAR. 
without altering the angle of the incident beam. This arrangement allows good statistics to be obtained for a minimum reflectivity of about $5 \times 10^{-7}$ in less than three hours. For time dependent experiments, reflectivities down to $5 \times 10^{-3}$ can be obtained in approximately $5 \mathrm{~min}$.

The sample stage $x-y-z-t h e t a-c h i$ goniometer, equipped with a sample changer, allows samples to be accurately positioned relative to the beam. SPEAR uses a single 3 He linear position-sensitive detector with $\sim 2 \mathrm{~mm}$ FWHM resolution for simultaneous studies of both specular and off-specular scattering.

\subsubsection{Optical Microscope (OM)}

In optical microscopes (OM), there are several methods that enhance the contrast of the fluctuations in the sample, such as bright field, dark field, polarized, differential interference, and phase contrast (PC) methods. Among these methods PCOM is the most sensitive to detect the in-plane local difference in the optical length of the sample [12]. The local difference in refractive index and/or thickness of the sample affect the optical length. Accordingly, the phase separation in thin film and the de-wetting phenomena can sensitively be detected by the PCOM. In this study NIKON OPTIPHOT-2 equipped with the phase contrast unit was used.

\subsubsection{Atomic Force Microscope (AFM) [12]}

AFM is a device to observe the height distribution on the surface of the sample using atomic force which works between probe and sample surface. The preparation for the sample is not needed in the AFM observation because the atomic force works without fail between probe and sample surface, and easily observe the insulating material. Many examples of observing AFM of the polymeric material have been widely reported from the conformation of single polymer to surface morphology of polymer blend. Concerning with scale, the object of observation is from single molecule to macroscopic materials of nonflatness with the level of $100 \mathrm{~nm}$.

Contact mode is to scan the sample surface in the state of contact with probe. The probe that is called a cantilever is attached to end point of the blade spring. The interaction between probe and surface of sample, specifically atomic force, are detected based on the amount of flexure of probe. The laser light is irradiated to the back of the cantilever, and the amount of flexure is evaluated by reflected light. Reflected light is 
detected by using photodiode division into four. Normally, feedback is put for constant power between probe and surface of sample, surface of sample is observed.

\subsubsection{Scanning Near-Field Optical Microscopy (SNOM) [13]}

In optical microscopes, the resolution is limited by the diffraction-limited, namely the order of wavelength of light, due to make it refract and diffract light with a lens.

Scanning near-field optical microscopy (SNOM) is a novel technique that breaks the optical diffraction barrier. As a member of the family of scanning probe microscopes, SNOM is equipped with a quasi-point light source that has a diameter much smaller than the wavelength of the excitation light. By scanning the sample surface at a very close distance, SNOM can reach a resolution down to tens of nanometers [14][15]. The advantage of SNOM over other microscopy techniques arises not only from its ability to create high resolution, spectrally resolved optical images that allows one to study the objects within nanometer scale, but also from its nature of operating with light. As a complement to other techniques such as scanning tunneling microscopy, scanning electron microscopy, and atomic force microscopy, SNOM is extremely useful in nanoscience related different optical properties, such as fluorescence, absorption, and polarization. In recent years, SNOM has been widely applied to many fields in polymer science such as studies on phase separation in polymer blend [16], single-chain characterizations in polymer monolayers [17], and investigation on the ordered structure of block copolymers [18, 19]. 


\section{References}

[1] Born M, Wolf E, Principles of Optics. Oxford: Pergamon Press; (1975).

[2] Lekner J, Theory of Reflection. Dordrecht, Martinus Nijhoff (1987).

[3] Russell T P, Materials Science Reports 5, 171 (1990).

[4] Thomas R K, Neutron reflection from polymer bearing surfaces. Scattering Methods in Polymer Science. R W Richards. London, Ellis Horwood (1995).

[5] Sears V F, Scattering lengths for neutrons. International Tables for Crystallograhy C.A.J.C. Wilson. Dordrecht, Kluwer (1992).

[6] Creagh D C, Hubbell J H, X-ray absorption coefficients. International Tables for Crystallograhy C.A.J.C. Wilson. Dordrecht, Kluwer (1992).

[7] Jones R L, Richards R W. Polymers at Surface and Interfaces. Cambridge: Cambridge University Press; (1999).

[8] Nishida K, Ogawa H, Matsuba G, Konishi T, Kanaya T, submitted to J. App. Crys.

[9] van de Hulst H C, Light Scattering by Small Particles, New York, John Wiley \& Sons (1957).

[10] Ferri F, Rev. Sci. Instrum. 68, 2265 (1997).

[11] Ebisawa T, Tasaki S, Otake Y, Funahashi H, Soyama K, Torikai N, Matushita Y, Physica B, 213\&214, 901 (1995).

[12] Nose T, Horie T, Kanaya T, A Laboratory Guide to Structure and Property Measurements of Organic Compounds and Polymers for Young Chemists, Kodansha Scientific (2006).

[13] Yang J, Sekine R, Aoki H, Ito S, Macromolecules 40, 7573 (2007).

[14] Betzig E, Trautman J K, Science 257, 189 (1992).

[15] Dunn R, Chem. Rev. 99, 2891 (1999).

[16] Aoki H, Kunai Y, Ito S, Yamada H, Matsushige K, Appl. Surf. Sci. 188, 534 (2002).

[17] Aoki H, Anryu M, Ito S, Polymer 46, 5896 (2005).

[18] Fasolka M J, Goldner L S, Hwang J, Urbas A M, DeRege P, Swager T, Thomas E L, Phys. Rev. Lett. 90, 016107 (2003).

[19] Raschke M B, Molina L, Elsaesser T, Kim D H, Knoll W, Hinrichs K, ChemPhysChem 6, 2197 (2005). 


\section{Chapter 3}

\section{Phase Separation and Dewetting in Polystyrene /Poly(vinyl methyl ether) Blend Thin Films in a Wide Thickness Range}

\subsection{Introduction}

It was reported that the structure and kinetics of phase separation in thin films of polystyrene (PS) and poly (vinyl methyl ether) (PVME) blend are very different from the bulk [1]. In addition, dewetting is also often observed in the thin films of the blends. Tanaka et al. [2] studied the surface structure of PS/PVME ultrathin films using atomic force microscopy (AFM) and observed dewetted droplets in one phase region for films thinner than twice the radius of gyration. Karim et al. [3, 4] also reported similar undulation of the free surface in the two phase region where dPS is encapsulated by PVME in the thin film. Small-angle light scattering studies on the kinetic of phase separation of PS/PVME blend thin films [5] show that the phase separation proceeds by a spinodal decomposition mechanism in a thick film (= $180 \mathrm{~nm})$ while in the thin film $(=30 \mathrm{~nm})$ it switches to nucleation and growth. These observations suggest that phase separation and dewetting competitively occur in thin films of the blends. However, there are no systematic studies on thin blend films of PS and PVME in a wide film thickness range.

In this Chapter, therefore, we investigated morphology and kinetics of phase separation as well as dewetting in thin films of PS/PVME blends as a function of film thickness using light scattering (LS) techniques and optical microscope (OM) as well as using atomic force microscopy (AFM) in a wide thickness $d$ range from semi-infinite thickness $(65 \mu \mathrm{m})$ to that close to radius of gyration $R_{\mathrm{g}}$ of PS and PVME chains $(d \sim$ $1.5 R_{\mathrm{g}}$ ) to elucidate the relation between phase separation and dewetting in blend thin films of PS and PVME in the two phase region.

\subsection{Experimental}

Polystyrene (PS) and poly(vinyl methyl ether) (PVME) used in this study have weight -average molecular weights $M_{\mathrm{w}}=280,000$ and 90,000, and the molecular weight 
distributions in terms of $M_{\mathrm{w}} / M_{\mathrm{n}}=3.01$ and 1.88, respectively, where $M_{\mathrm{n}}$ is number -average molecular weight. Both of PS and PVME were purchased from Scientific Polymer Products, Inc. PS and PVME were purified by precipitating the toluene solutions into excess methanol and $n$-heptane several times, respectively, and dried in vacuum at room temperature for $72 \mathrm{~h}$. It is noted that PS and PVME used in this experiment have similar values of radius of gyration $R_{g}$, which are $16.6 \mathrm{~nm}$ and $16.2 \mathrm{~nm}$, respectively, although their molecular weights are different. Films below and above 1 $\mu \mathrm{m}$ were prepared by spin-coating and casting the toluene solution of PS and PVME, respectively, on a cleaned glass substrate after filtering with $2 \mu \mathrm{m}$ pore size membrane at room temperature. The films were then annealed at $60{ }^{\circ} \mathrm{C}$ for $24 \mathrm{~h}$ after drying in vacuum at room temperature for $24 \mathrm{~h}$. Thickness of polymer film was controlled by varying the polymer concentration in solution and confirmed with ellipsometer measurements.

Small-angle light scattering (LS) measurements were carried out using home-made apparatus with confocal collimation, which enables us to access a very low $q$ range down to $0.1 \mu \mathrm{m}^{-1}$ with extremely low background. These characteristic features enable us to measure an extremely thin film. Two dimensional scattering intensities were accumulated every 5 min after temperature jump from one phase region to two phase region $\left(=115{ }^{\circ} \mathrm{C}\right)$. Details of the LS instrument were reported in Chapter 2 [6]. Optical microscope (OM) measurements were also done after temperature jump from one phase region to two phase region $\left(=115^{\circ} \mathrm{C}\right)$ using OLYMPUS BX50 equipped with a CCD camera. Atomic force microscope (AFM) measurements were performed at room temperature after quenching the sample from two phase region $\left(=115^{\circ} \mathrm{C}\right)$ using JEOL JSPM-4200 to examine the surface morphology of the films.

\subsection{Results and Discussion}

First of all we have constructed a phase diagram of the bulk PS/PVME blend. We observed LS intensity from samples with various blend ratios during the heating process from room temperature with a rate of $1^{\circ} \mathrm{C} / \mathrm{min}$. It was reported [7] that the phase diagram dose not depend on the heating rate when it is below $2{ }^{\circ} \mathrm{C} / \mathrm{min}$. In this measurement the cloud point was defined as an onset temperature of the scattering intensity. The phase diagram thus obtained is shown in Figure 3.1, which is a lower critical solution temperature (LCST) type. The estimated critical weight fraction of PS 


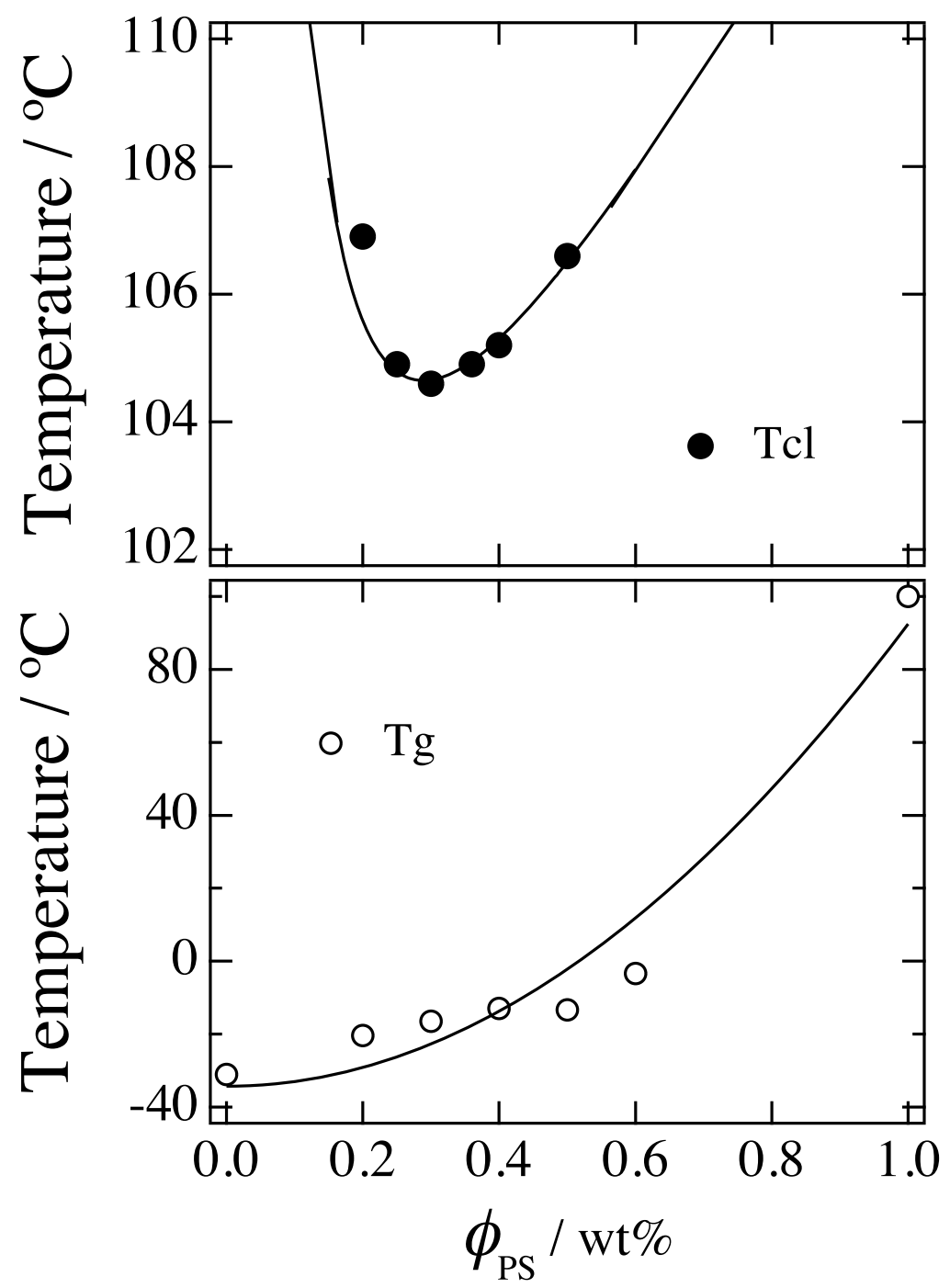

Figure 3.1: Phase diagram (top) and the glass transition temperature $T_{\mathrm{c}}$ (bottom) of PS/PVME blend in bulk. 
$\phi_{\mathrm{PS}}$ is 0.3 and the critical temperature $T_{\mathrm{C}}$ is $104.6{ }^{\circ} \mathrm{C}$. At $115{ }^{\circ} \mathrm{C}$ the coexisting composition are nearly pure components, which were estimated by extrapolating the phase diagram in Figure 3.1. The glass transition temperature $T_{\mathrm{g}}$ of the bulk blend has been estimated as a function of the concentration, which is shown in Figure 3.1. The $T_{\mathrm{g}}$ of the blend with the critical concentration $\left(\phi_{\mathrm{PS}}=0.3\right)$ is $-20^{\circ} \mathrm{C}$. The phase diagram is almost identical to the reported one [8]. In this chapter all LS, OM and AFM measurements were performed on PS/PVME blend thin films with the critical concentration of $\phi_{\mathrm{PS}}=0.3$.

OM measurements were performed on the blend thin films as a function of annealing time after temperature jump to $115{ }^{\circ} \mathrm{C}$ in two phase region from one phase region. In addition the surface morphology was examined by AFM with contact mode after quenching the film to room temperature after $90 \mathrm{~min}$ annealing at $115{ }^{\circ} \mathrm{C}$. The OM images are shown in Figure 3.2 for $65 \mu \mathrm{m}, 9 \mu \mathrm{m}, 2 \mu \mathrm{m}, 466 \mathrm{~nm}, 98 \mathrm{~nm}$ and $42 \mathrm{~nm}$ films at 20, 50, 90 and 120 min after the temperature jump, and the AFM results are given in Figure 3.3. In the following we will discuss the OM and AFM results in order of the film thickness.

In the case of $65 \mu \mathrm{m}$ film, the observed OM patterns look like typical SD phase separation process although they are 2D images, and the AFM results show the surface is very smooth. These observations suggest that the SD type phase separation proceeds inside the $65 \mu \mathrm{m}$ film. For the 9 and $2 \mu \mathrm{m}$ films similar SD patterns are observed at 20 min in the early stage although the characteristic wavelengths seem smaller than that in the $65 \mu \mathrm{m}$ film. The surfaces of the 9 and $2 \mu \mathrm{m}$ films are very smooth, at least at 20 min, which was confirmed by AFM measurements. As the phase separation proceeds some semi-irregular spot-like patterns with strong (white) contrast are observed over the SD pattern at 90 and $120 \mathrm{~min}$ in the later stage. This is well recognized in the OM image at $120 \mathrm{~min}$ for the $2 \mu \mathrm{m}$ film (Figure 3.2(c)). The AFM result in Figure 3.3(b) clearly shows that the white spots are holes on the surface, confirming that the semi-irregular pattern is due to dewetting.

In the $466 \mathrm{~nm}$ film, the observed OM images are so irregular even at 20 and $50 \mathrm{~min}$ and grow with annealing time keeping the irregularity as seen in Figure 3.2(d). The surface morphology examined by AFM confirmed that the irregular pattern was due to irregular dewetting morphology. As the film thickness further decreases, the time evolution of the OM and AFM images very much changed for the 98 and $42 \mathrm{~nm}$ films. 


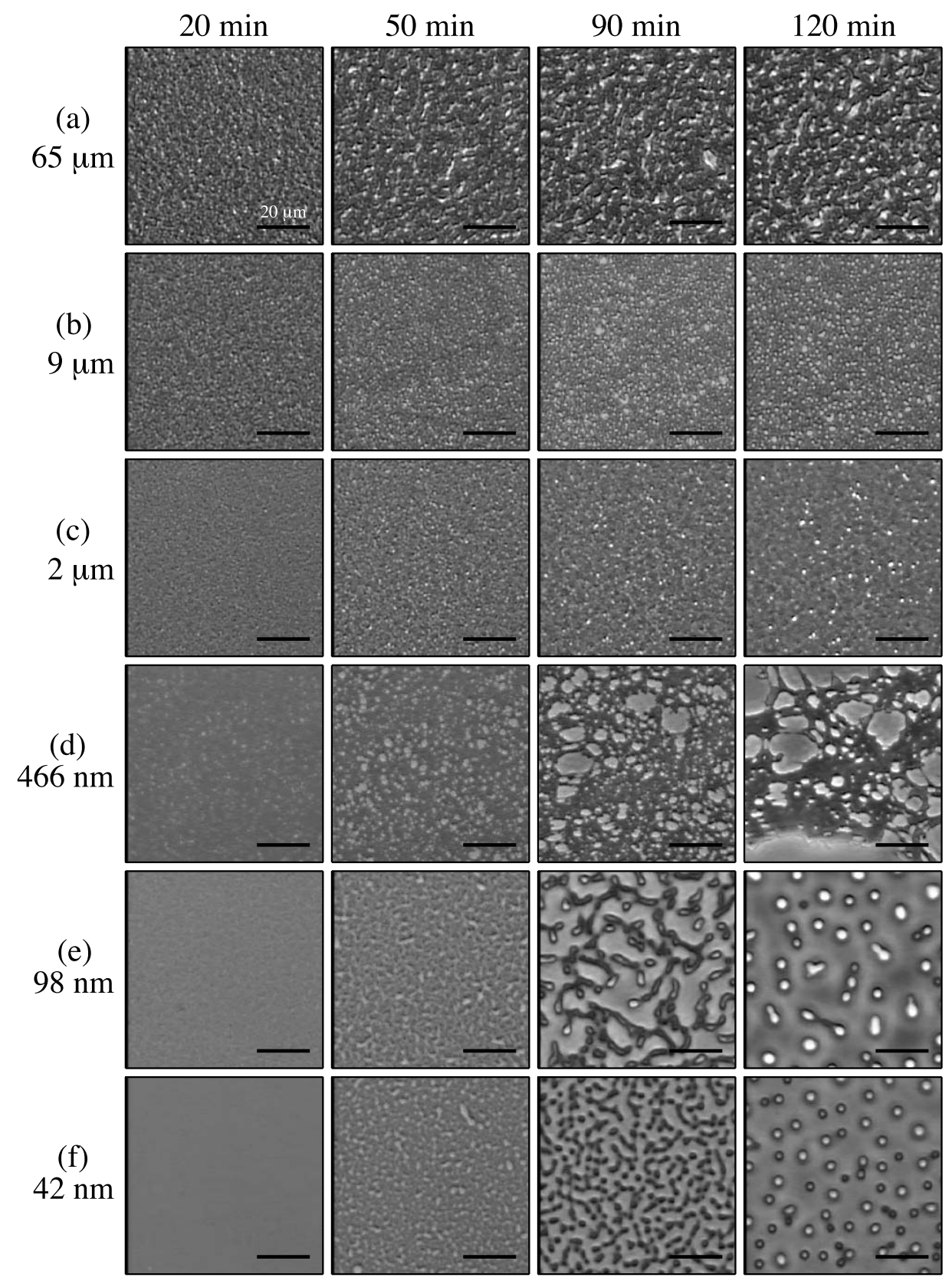

Figure 3.2: Time evolution of $\mathrm{OM}$ images after temperature jump to $115{ }^{\circ} \mathrm{C}$ in two phase region from one phase region for film thickness (a): $65 \mu \mathrm{m}$, (b): $9 \mu \mathrm{m}$, (c): $2 \mu \mathrm{m}$, (d): $466 \mathrm{~nm}$, (e): $98 \mathrm{~nm}$ and (f): $42 \mathrm{~nm}$. Scale bars in OM images are $20 \mu \mathrm{m}$. 


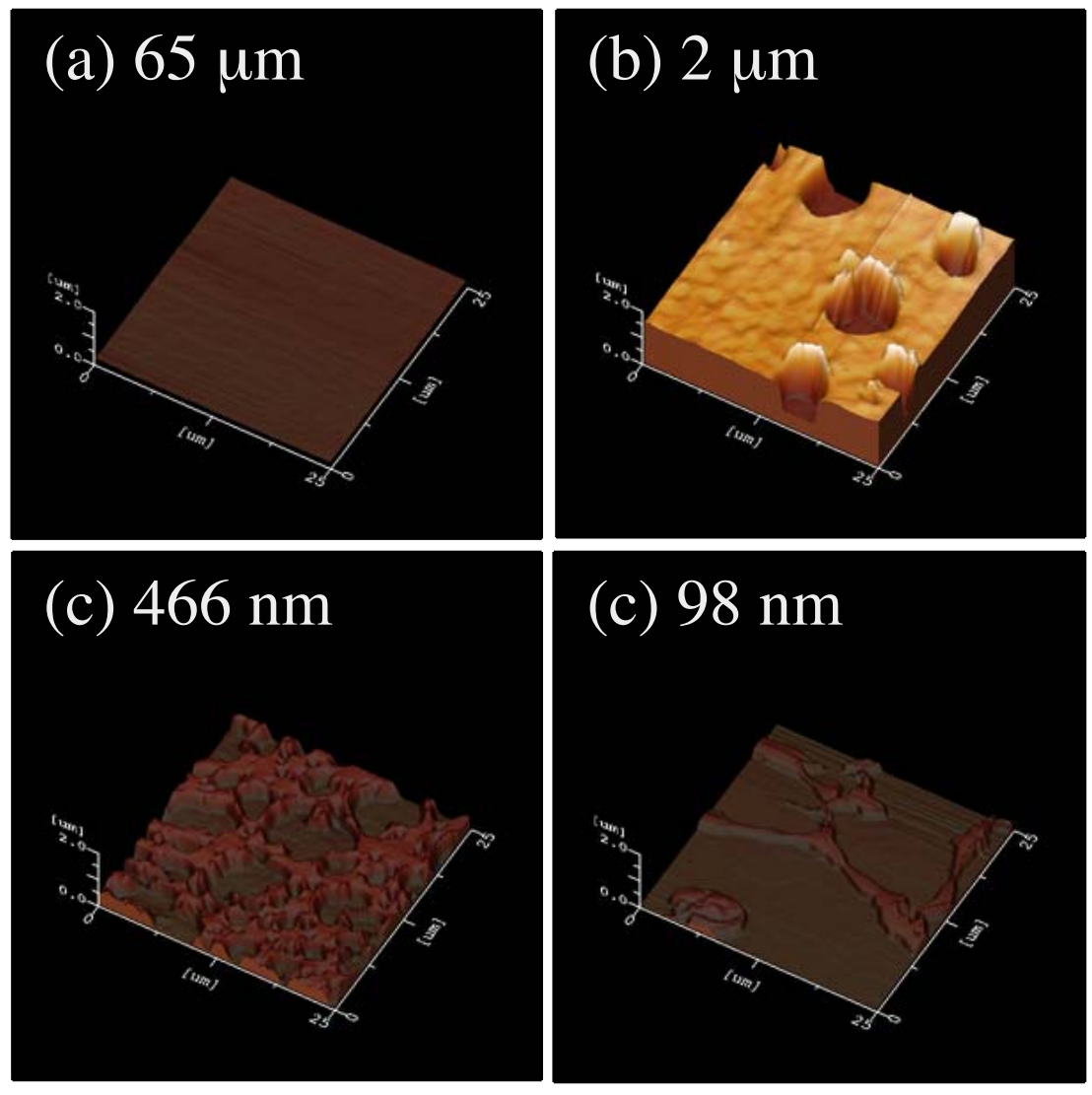

Figure 3.3: AFM images of PS/PVME blend thin films quenched to room temperature after annealing at $115{ }^{\circ} \mathrm{C}$ in two phase region. (a): $65 \mu \mathrm{m}$ after $90 \mathrm{~min}$ annealing, (b): $2 \mu \mathrm{m}$ after $120 \mathrm{~min}$ annealing, (c): $466 \mathrm{~nm}$ after $90 \mathrm{~min}$ annealing and (d): $98 \mathrm{~nm}$ after $90 \mathrm{~min}$ annealing. Z-range is $2.0 \mu \mathrm{m}$ and $\mathrm{x}-\mathrm{y}$ size is $25 \mu \mathrm{m} \times 25$ $\mu \mathrm{m}$ for all images. 
In the first 20 or $30 \mathrm{~min}$ the $\mathrm{OM}$ images are very homogeneous and the surfaces examined by AFM are very smooth, implying nothing happens. After the long incubation period regular bicontinuous morphologies with strong contrast are observed in the OM images, and finally become isolated droplets at $120 \mathrm{~min}$. The AFM images of the $98 \mathrm{~nm}$ after $90 \mathrm{~min}$ annealing at $115^{\circ} \mathrm{C}$ in Figures 3.3(d) clearly shows that the regular pattern is due to the dewetting. The present OM and AFM results show that the phase separation as well as dewetting in the PS/PVME blend thin film depend on the film thickness very much.

Concerning the dewetting in the blend thin films, it is emphasized that dewetting in one component (PS or PVME) films dose not occur in such thick films as examined here. For example, dewetting occurs in PS films below about 5-6 nm [9] while it depends on temperature and molecular weight more or less. In any case, such thick one component films as $470 \mathrm{~nm}$ do not show dewetting. We also studied the stability of the blend films in a range from $25 \mathrm{~nm}$ to $65 \mu \mathrm{m}$ at $90{ }^{\circ} \mathrm{C}$ in one phase region and found that no dewetting was observed during annealing at $90{ }^{\circ} \mathrm{C}$ for $6 \mathrm{~h}$ even in the 25 $\mathrm{nm}$ film, showing that the blend films are stable in one phase region. The present results suggest that dewetting observed in the PS/PVME thin films is induced by the phase separation.

In the next step, we studied the phase separation and dewetting of the PS/PVME blend thin films using time-resolved LS just after temperature jump to $115^{\circ} \mathrm{C}$ in two phase region from one phase region. Figures 3.4(a)-(f) show the time evolutions of one dimensional (1D) LS profiles for the $65 \mu \mathrm{m}, 9 \mu \mathrm{m}, 2 \mu \mathrm{m}, 466 \mathrm{~nm}, 98 \mathrm{~nm}$ and 42 $\mathrm{nm}$ films, which were obtained by circular averaging the $2 \mathrm{D}$ scattering profiles. The scattering profiles and their time evolutions clearly depend on the film thickness as observed in the OM and AFM measurements. In the $65 \mu \mathrm{m}$ film the scattering profile shows a peak and its position gradually shifts to lower $q$ with annealing time, showing typical spinodal decomposition (SD) type phase separation kinetics. In a rather thin film such as $2 \mu \mathrm{m}$, however, the time evolution of the scattering profile is different. In an early stage before $\sim 30 \mathrm{~min}$ the time evolution of the scattering profile is very similar to that in the $65 \mu \mathrm{m}$ film, however, the growth in the peak intensity is slowed down and the intensity in a low $q$ range below the peak grows in a late stage after $\sim 60 \mathrm{~min}$. This must be caused by the semi-irregular holes on the surface due to the dewetting as seen in OM images (see Figures 3.2(b) and (c)). 


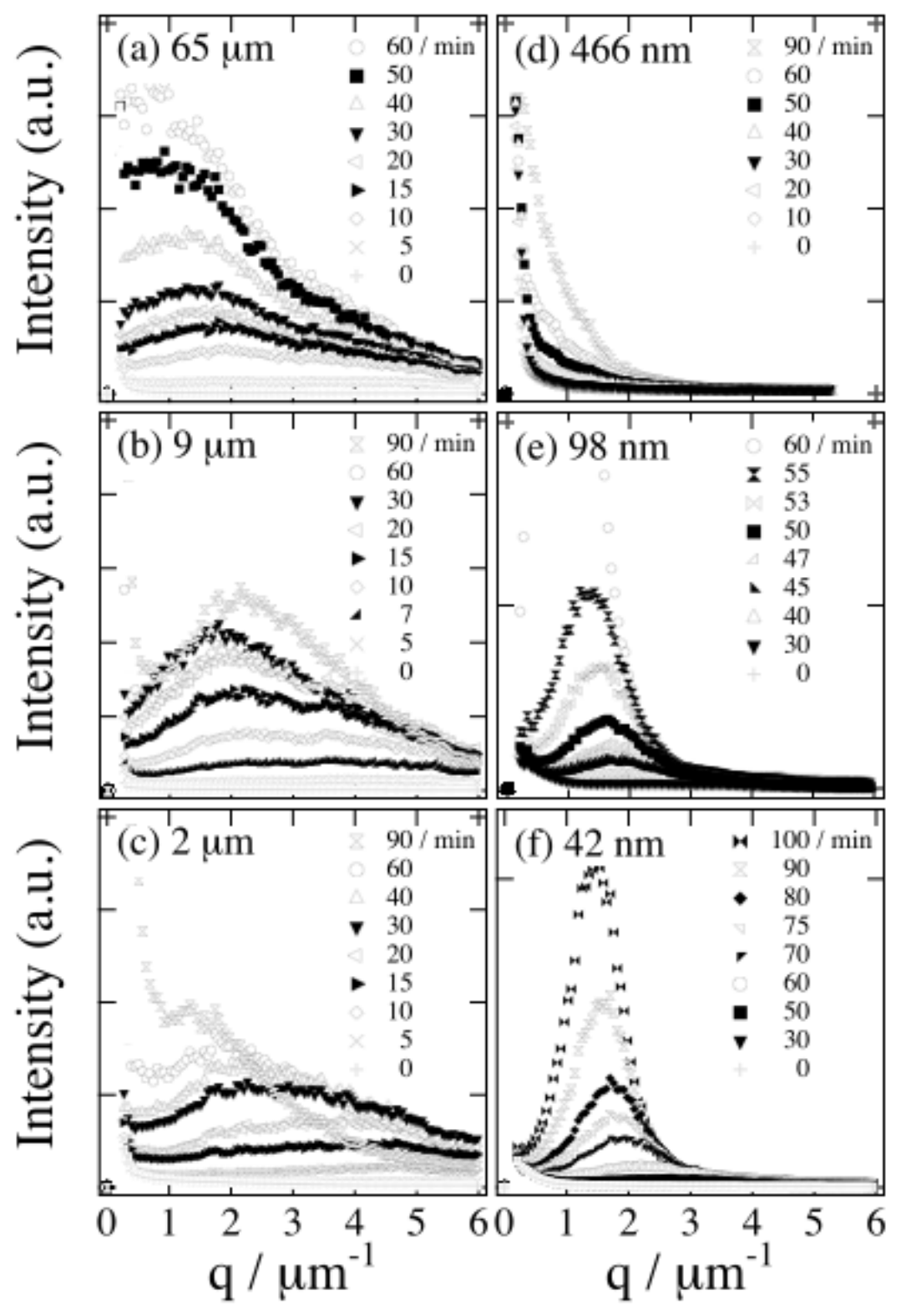

Figure 3.4: Time evolution of LS profiles after temperature jump to $115{ }^{\circ} \mathrm{C}$ in two phase region from one phase region for film thickness (a): $65 \mu \mathrm{m}$, (b): 9 um. (c): 2 um. (d): 466 nm. (e): $98 \mathrm{~nm}$ and (f): 42 nm. 
As the film thickness further decreases, the scattering peak disappears for films below $\sim 1 \mu \mathrm{m}$ as seen for the $466 \mathrm{~nm}$ film in Figure 3.4(d). This must correspond to the irregular dewetting morphology observed in the OM and AFM measurements (see Figure 3.2(d) and Figure 3.3(c)). The scattering pattern obtained by fast Fourier transform (FFT) of the OM image of the $466 \mathrm{~nm}$ film do not show any peak in the low $q$ range down to $0.04 \mu^{-1}$, confirming that there are not characteristic wavelengths in the spatial scale below $\sim 150 \mu \mathrm{m}$. With further decreasing the film thickness, surprisingly, the peak appears again below $200 \mathrm{~nm}$ and the incubation time before appearing the peak is very long.

For quantitative discussions we plotted the peak intensity $I_{\max }$ and the peak position $q_{\max }$ as a function of annealing time in Figures 3.5(a) and (b), respectively, for the 65 $\mu \mathrm{m}, 14 \mu \mathrm{m}, 9 \mu \mathrm{m}, 2 \mu \mathrm{m}, 98 \mathrm{~nm}$ and $42 \mathrm{~nm}$ films. We first examine the results of the $65 \mu \mathrm{m}$ film. The scattering peak appears at around $2 \mu \mathrm{m}^{-1}$ at $\sim 7 \mathrm{~min}$ after the temperature jump and the scattering intensity exponentially increases with annealing time before $\sim 7$ min. After $\sim 7$ min, as can be seen in Figures 3.5(a) and (b), the peak intensity $I_{\max }$ and the peak position $q_{\max }$ increase according to power laws $I_{\max }(t) \sim t^{\beta}$ and $q_{\max }(t) \sim t^{-\alpha}$, respectively, suggesting that the scaling relations are hold. At around 30 min the slopes in $q_{\max }(t) \sim t^{-\alpha}$ and $I_{\max }(t) \sim t^{\beta}$ become steeper. The exponents $\alpha$ and $\beta$ evaluated from the observed time evolutions of $I_{\max }$ and $q_{\max }$ are $\alpha=0.25$ and $\beta=0.85$ before $30 \mathrm{~min}$ and $\alpha=0.54$ and $\beta=1.62$ after $30 \mathrm{~min}$, respectively, for the $65 \mu \mathrm{m}$ film. These values are very close to those reported for the PS/PVME bulk blend by Hashimoto et al. $[10,11]$, and the relations $\beta>3 \alpha$ and $\beta=3 \alpha$ are hold before and after $30 \mathrm{~min}$, respectively. According to Hashimoto et al. the former and the latter can be regarded as the intermediate stage and the late stage of spinodal decomposition (SD) type phase separation, respectively [11]. From these LS results, we concluded that the $65 \mu \mathrm{m}$ film could be regarded as a bulk sample. As mentioned above, we did not observe a scattering peak in the very early stage before $\sim 7 \mathrm{~min}$. This was also observed in the experiments by Hashimoto et al. [10, 11].

The phase separation kinetics is almost independent of the film thickness for the films above $\sim 15 \mu \mathrm{m}$. This region is termed Region I hereafter. On the other hand, as the film thickness decreases below $\sim 15 \mu \mathrm{m}$, effects of the film thickness gradually appear on the kinetics. In the case of $14 \mu \mathrm{m}$ film, the phase separation kinetics seems to be identical with that in the $65 \mu \mathrm{m}$ film (bulk), at least, until 20 min after the 


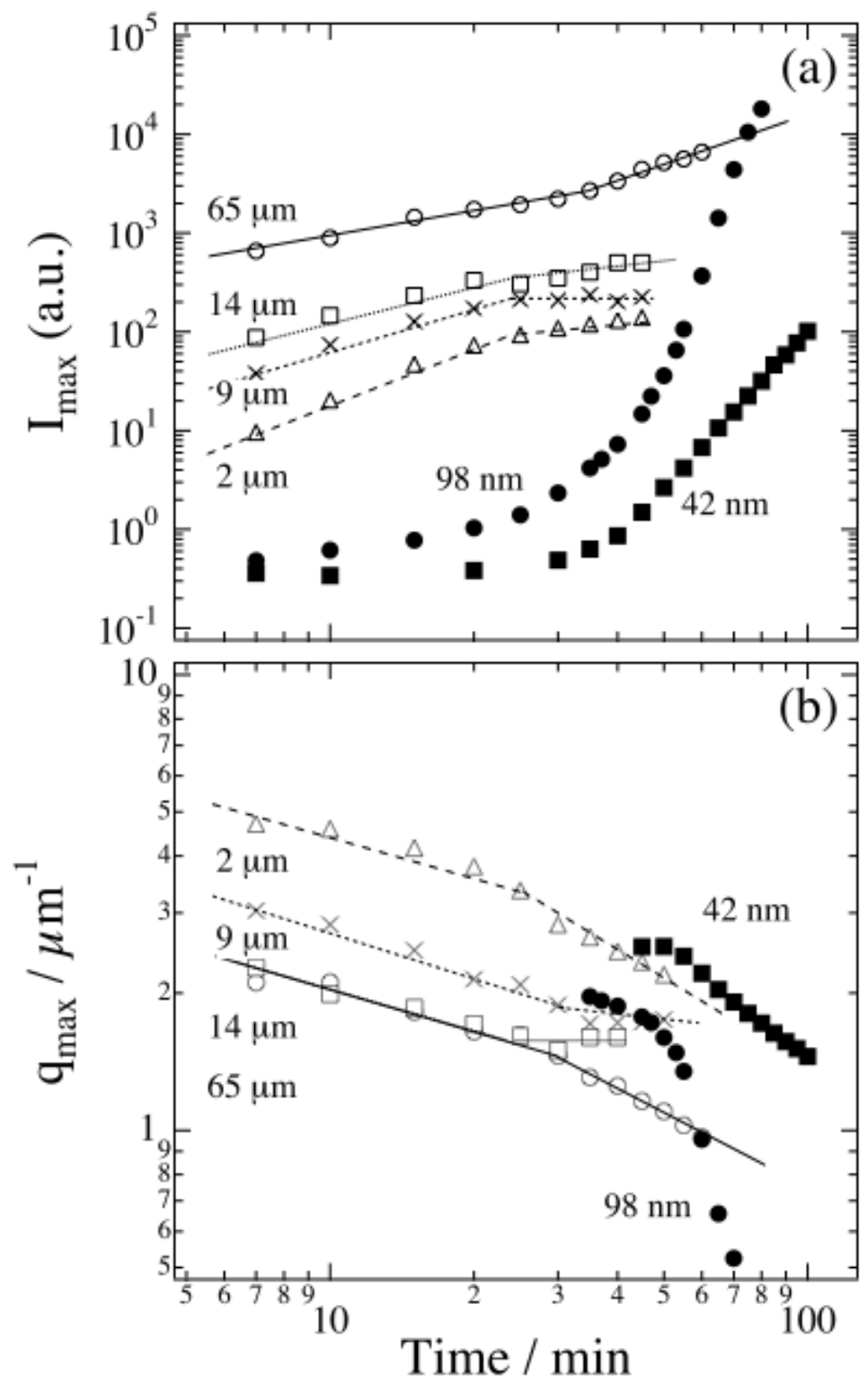

Figure 3.5: Time evolution of (a) peak intensity $I_{\max }$ and (b) peak position $q_{\max }$ for film thickness $65 \mu \mathrm{m}, 14 \mu \mathrm{m}, 9 \mu \mathrm{m}, 2$ $\mu \mathrm{m}, 98 \mathrm{~nm}$ and $42 \mathrm{~nm}$. 
temperature jump as seen in Figure 3.5. However, they deviate from the bulk behavior at $\sim 20 \mathrm{~min}$, showing some effects of film thickness. For films below $\sim 15 \mu \mathrm{m}$ and above $\sim 1 \mu \mathrm{m}$, we observed similar level-off of the peak intensity after $\sim 20 \mathrm{~min}$. This region is termed Region II.

In order to see the differences between Region I and Region II, we plotted the characteristic wavelength $\lambda_{\max }\left(=2 \pi / q_{\max }\right)$ at 10 min after the temperature jump as a function of the film thickness in Figure 3.6. In the figure the characteristic wavelength for the films below $200 \mathrm{~nm}$ is also plotted, which will be discussed later. The characteristic wavelength $\lambda_{\max }$ is almost constant for the films above $\sim 15 \mu \mathrm{m}$ (Region I), however, it begins to decrease with film thickness between $\sim 15$ and $\sim 1 \mu \mathrm{m}$ (Region II). When the characteristic wavelength $\lambda_{\max }$ encounters the film thickness $d$ (thick solid line in Figure 3.6) the characteristic peak disappears in the scattering profile, suggesting cut-off of the fluctuations with longer wavelength than the film thickness. In Figure 3.7, the LS profiles at $10 \mathrm{~min}$ in the early stage after temperature jump are shown for the 2, 4 and $65 \mu \mathrm{m}$ (bulk) films, the intensities of which were normalized to that at $q=5$ $\mu \mathrm{m}^{-1}$ to compare the shapes of profiles. In the figure, $q$ values corresponding to the film thickness $(q=2 \pi / d)$ are indicated by down arrows. It is very clear that the scattering intensity in a $q$ range lower than the scattering vector corresponding to the film thickness is suppressed, meaning that the fluctuations with wavelength longer than the film thickness are hard to appear in the thin films.

As the film thickness further decreases below $\sim 1 \mu \mathrm{m}$, the scattering profiles are very different from those above $\sim 1 \mu \mathrm{m}$. As demonstrated for the $466 \mathrm{~nm}$ film (see Figure 3.4(d)), no scattering peak is observed during the time evolution in the film thickness range between $\sim 1 \mu \mathrm{m}$ and $\sim 200 \mathrm{~nm}$, which is termed Region III. As shown in the OM and AFM measurements (Figure 3.2(d) and Figure 3.3(c)) this is due to the irregular dewetting morphology. However, at the moment we have no final conclusions on the irregular pattern. One possibility is that the phase separation and dwetting occur simultaneously and their characteristic wavelengths are mixed up, resulting in the irregular morphology.

For the films below 200 nm (Region IV) a scattering peak appears again (see Figure 3.2). The time evolutions of the peak intensity $I_{\max }$ and the peak position $q_{\max }$ are shown for the $98 \mathrm{~nm}$ and $42 \mathrm{~nm}$ films in Figures 3.5(a) and (b). This characteristic wavelength $\lambda_{\max }\left(=2 \pi / q_{\max }\right)$ is due to the dewetting as confirmed in the AFM 


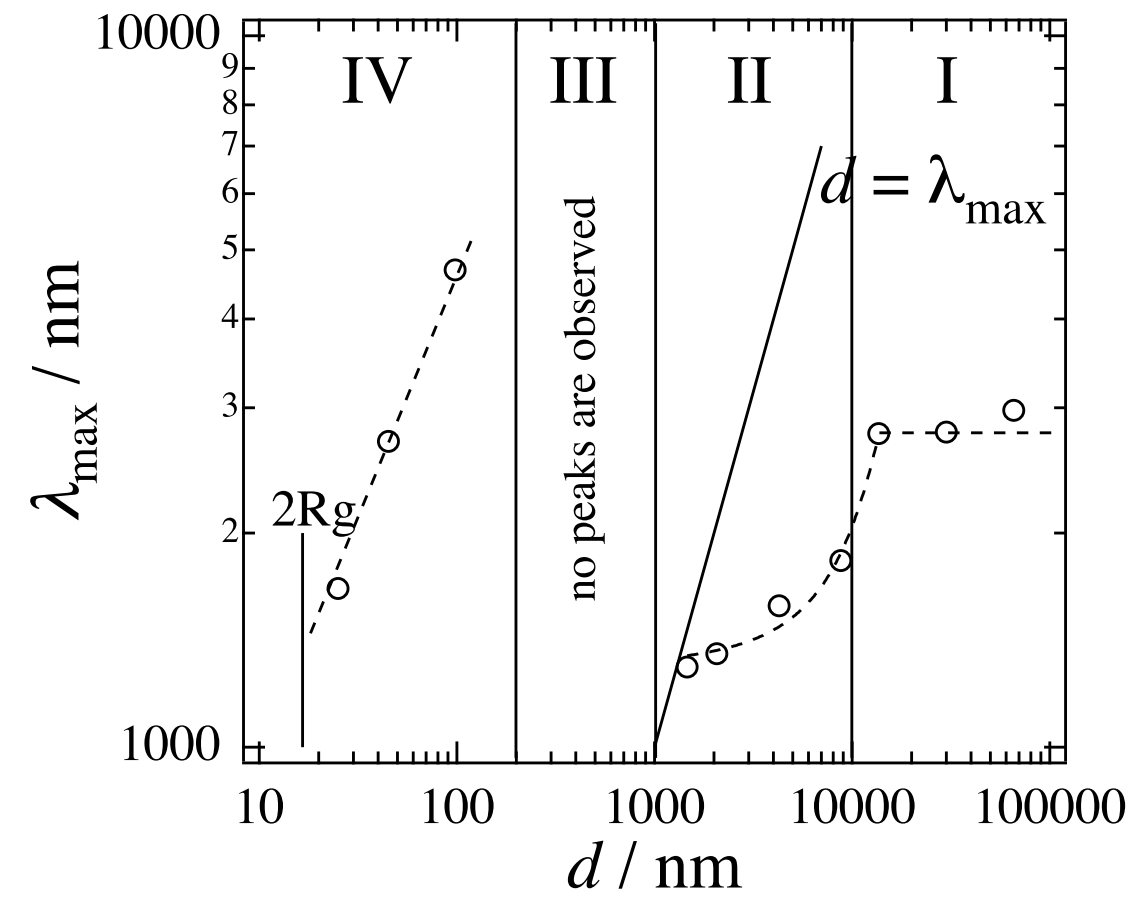

Figure 3.6: Characteristic wavelength $\lambda_{\max }$ at $10 \mathrm{~min}$ (for Region I and II) and at $50 \mathrm{~min}$ (for Region IV) after temperature jump to $115^{\circ} \mathrm{C}$ in two phase region from one phase region as a function of film thickness. In Region I and II, a thick solid line shows a relation that film thickness $d$ is equal to characteristic wavelength $\lambda_{\max }(d=$ $\lambda_{\max }$. 


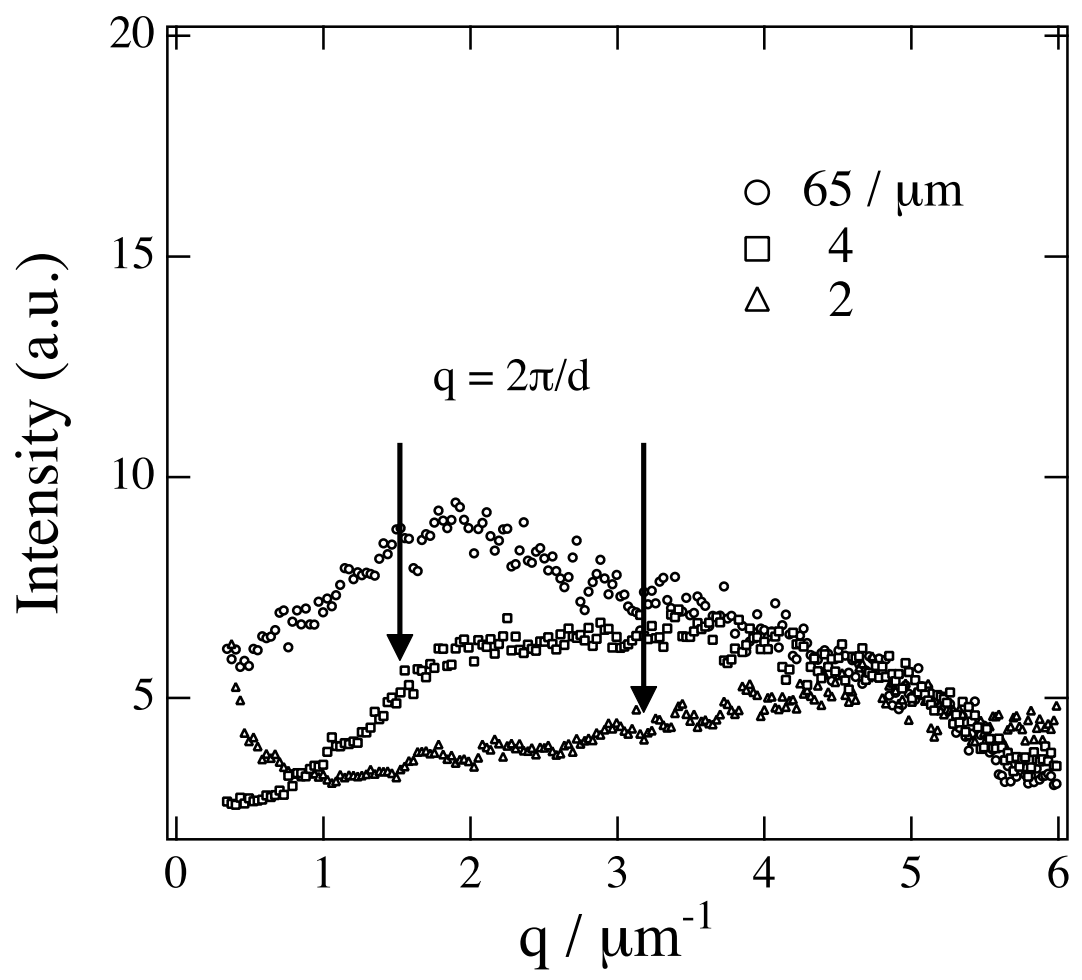

Figure 3.7: LS profiles in the early stage of phase separation for the $65 \mu \mathrm{m}, 4 \mu \mathrm{m}$ and $2 \mu \mathrm{m}$ films. The intensity is normalized to that at $q=5 \mu \mathrm{m}^{-1}$. 
measurements. The characteristic wavelength of dewetting in the early stage for the $98 \mathrm{~nm}$ film is larger than that of the SD phase separation in the films above $\sim 15 \mu \mathrm{m}$ (Figure 3.6), and decreases with the film thickness. This is one of the characteristic features of dewetting in the PS/PVME thin films below 200 nm (Region IV). Another characteristic feature is a very long incubation time before appearance of the scattering peak, which are $\sim 30 \mathrm{~min}$ and $\sim 40 \mathrm{~min}$ for the $98 \mathrm{~nm}$ and $42 \mathrm{~nm}$ films, respectively. During the incubation period the scattering intensities hardly grow, while they increase very rapidly after the incubation period (see Figure 3.5(b)) according to a power law with unexpected large exponents. In the following we will discuss the dewetting mechanism in Region II and IV.

In the region II, for example, we consider the $2 \mu \mathrm{m}$ film. The scattering peak was observed at around $q_{\max }=5 \mu \mathrm{m}^{-1}$ in the early stage of phase separation and gradually shifted to lower $q$ with annealing time (Figure 3.5(b)). When $q_{\max }$ comes close to the film thickness ( $q=2 \pi / 2 \sim 3 \mu \mathrm{m}^{-1}$ ) at $\sim 25 \mathrm{~min}$, the growth of scattering intensity slows down or stops. Similar tendency was observed in the 9 and $14 \mu \mathrm{m}$ films in Region II. From the OM results, it was also observed that the growth of phase separation stopped and the dewetting began to occur. These observations show that the composition fluctuations due to the SD phase separation induce the in-plane fluctuations on the surface/interface and rupture the film. An important problem we have to clarify is how the SD composition fluctuations induce the dewetting. Recently Chung et al. [12] have shown that phase separation drove dewetting in blend films of poly(methyl methacrylate) (PMMA) and poly(styrene-ran-acrylonitrile) (SAN) ( $d=550 \mathrm{~nm}$ ), and also shown that the capillary fluctuations failed to explain the roughening (or dewetting). On the basis of a universal scaling between the long wavelength fluctuations and surface roughness, they proposed that spinodal decomposition during initial phase separation may trigger the long wavelength fluctuations, and pointed out a possibility that the long wavelength fluctuations are created through the composition fluctuation mechanism proposed by Clarke [13]. In Region II, thus the dewetting induced by the SD composition fluctuations is one of the candidates.

In Region IV, the regular patterns in the dewetting process suggest that the dewetting process proceeds through the SD mechanism. Generally speaking, however, SD type dewetting occurs when the system is in an unstable region and no incubation period is required $[14,15]$. The long incubation time means that some structure is 
formed before the dewetting in the thin film and the dewetting may be triggered by the structure. In the incubation period we see the very small increase in the LS intensity (see Figure 3.5(a)) although it is not the SD type phase separation observed in Region II. This may suggest that some composition fluctuations proceed along the normal direction to the surface to form some layer structure, which are hard to be seen in the present scattering geometry with incident beam normal to the film surface. Such layer structure was reported for other polymer blend thin films. Stamm et al. [16] studied dewetting and phase separation in thin films of a weakly imcompatible polymer blend of deuterated polystyrene (dPS) and poly(p-methylstyrene) (PpMS), and found that the interactions with substrate during the phase separation leaded to a bi-layer formation with PpMS on the top layer. The bi-layer structure is unstable and defines the starting point for the dewetting on the top of the dPS layer. In the final dewetting state a homogeneous layer of dPS on the top of the substrate is covered with ultra-thin PpMS layer as well as the quite thick mesoscopic drops of PpMS. Apparently this mechanism is similar to our case. However, we observed bear Si surface in the AFM measurements, and hence no homogeneous layer of PVME was suggested. Blend thin films of deuterated poly(methyl methacrylate) (dPMMA) and poly(styrene-ran-acrylonitrile) (SAN) were also extensively studied by Composte et al. [17-20] in a wide thickness $d$ range from semi-infinite limit to less than radius of gyration $R_{\mathrm{g}}$ of a polymer chain. In the semi-infinite limit they observed the so-called surface directed spinodal decomposition (SDSD), which is suppressed when the characteristic length is below the film thickness. In the thin film regions of $10 R_{\mathrm{g}}<d<$ $150 R_{\mathrm{g}}$ and of $\sim R_{\mathrm{g}}<d<10 R_{\mathrm{g}}$, tri-layer structure is formed in the early stage, consisting of surface and interface dPMMA-rich layers and middle SAN-rich layer. In the SAN-rich middle layer they observed phase separated structure and homogeneous structure for the former and the latter, respectively. These reported results tell us that layered structure is easily formed due to the interactions between one of blend component polymer and substrate (and/or air), and triggered the dewetting. We therefore expect tri-layer structure formation due to the preferential interactions between PVME and substrate at the interface and between PVME and air at the surface [21] before dewetting and the preliminary result of neutron reflectivity (NR) measurements on the deuterated PS/PVME blend thin films supported the tri-layer structure [22]. Note that the surface and interface segregation of PVME reduces the 
concentration of PVME in the middle bulk-like layer, but the preliminary NR results suggest that it is negligible for the films above $\sim 50 \mathrm{~nm}$ [22].

For dewetting, the thin film must be ruptured by some mechanism. It was reported for dPMMA/SAN blend thin films $\left(10 R_{\mathrm{g}}<d<150 R_{\mathrm{g}}\right)$ [20] that the lateral phase coarsening occurs in the middle stage after the tri-layer structure formation, and finally the capillary fluctuations causes the thickness to vary and rapture the middle layer. Assuming the tri-layer structure formation in the PS/PVME blend thin film below 200 $\mathrm{nm}\left(\sim 13 R_{\mathrm{g}}\right)$ before dewetting, the situation is very similar to the dPMMA/SAN blend thin film. According to Composto et al. [19] the characteristic wavelength of the capillary fluctuations $\Lambda_{m}$ is given by

$$
\Lambda_{m}=h_{0}^{2}\left[4 \pi\left(\gamma_{1}+\gamma_{12}\right) / A\right]^{1 / 2}
$$

for tri-layer structure. Here, $h_{0}$ is the initial thickness of the middle layer, $\gamma_{1}$ and $\gamma_{12}$ are the surface and interfacial tensions of the top layer, and $A$ is the Hamaker constant. Eq. (1) predicts that the characteristic wavelength of the dewetting decreases with the film thickness. This agrees with our observation shown in Figure 5 although the detailed comparison is not impossible at the moment.

Recently An and co-workers [23] have explained the dewetting of thermodynamically stable blend ultrathin film of PMMA and SAN ( $d \sim R_{\mathrm{g}}$ ) in terms of a dewetting mechanism induced by composition fluctuations [23-26], which is a similar mechanism proposed in Region II This mechanism may be possible even in two phase region. PVME has attractive interactions with Si substrate and air [21], and hence would diffuse to the substrate so as to create the composition gradient across the film. The diffusion could not occur homogeneously over the film surface to create the composition fluctuations in the mixture along the surface. When the amplitude of the fluctuations is large enough, the free surface is eventually destabilized, leading to the dewetting. At the moment we have no direct evidence for the dewetting mechanism in Region IV, but cannot deny both of them as a rapture mechanism for blend thin film. Investigation in the composition fluctuations normal to the surface would be give us significant information on the dewetting mechanism.

\subsection{Conclusion}

In this chapter we investigated the morphology and the kinetics of phase separation 
and dewetting in blend thin films of PS/PVME as a function of film thickness from 40 $\mathrm{nm}$ to $65 \mu \mathrm{m}$ using OM and AFM as well as LS. The time evolutions of OM images and LS patters after the temperature jump into the two phase region strongly depend on the film thickness, and could be classified into four regions (Regions I, II, III and IV). In Region I above $\sim 15 \mu \mathrm{m}$, the phase separation kinetics can be explained as normal SD phase separation in bulk. As the film thickness decreases, the characteristic wavelength to the SD phase separation becomes smaller with the film thickness in Region II between $\sim 15$ and $\sim 1 \mu \mathrm{m}$ because the composition fluctuations are suppressed by the film thickness. In Region II the films finally dewet in a very late stage, depending on the film thickness, which must be induced by the phase separation. In Region IV below $\sim 200 \mathrm{~nm}$ the films dewet after a long incubation time. The bicontinuous morphologies with characteristic wavelength $\lambda_{\max }$ implies that the dewetting mechanism is a SD type, and the long incubation time was assigned to tri-layer structure formation. In Region III $(1 \mu \mathrm{m} \sim 200 \mathrm{~nm})$ between Region II and Region IV the LS patterns do not show any characteristic scattering peaks, corresponding to the irregular dewetting pattern confirmed by AFM. It suggests that the phase separation and dewetting occur simultaneously and their characteristic wavelengths are mixed up, resulting in the irregular morphology. In Regions II and IV the dewetting mechanisms were discussed. In Region IV the dewetting induced by the SD composition fluctuations was proposed. In Region IV below $\sim 200 \mathrm{~nm}$ preferential interactions between one component of blend polymers (PVME) and substrate as well as air lead to vertical phase separation to create tri-layer structure, consisting of interface and surface PVME layers and the middle blend layer. For dewetting, the middle layer must be ruptured by some mechanism. One possibility is the capillary fluctuations and the other is the composition fluctuations at the interlayer. This is examined in Chapter 5 by neutron reflectivity measurements. 


\section{References}

[1] Reich S, Cohen Y, J. Polym. Sci.: Polym. Phys. Ed. 19, 1255 (1981).

[2] Tanaka K, Yoon J S, Takahara A, Kajiyama T, Macromolecules, 28, 934 (1995).

[3] Ermi B D, Karim A, Douglas J F, J. Poly. Sci., Part. B, 36, 191 (1998).

[4] Karim A, Slawecki T M, Kumar S K, Douglas J F, Satija S K, Han C C, Russell T P, Liu Y, Overnay R, Sokolov J, M. H. Rafailovich, Macromolecules, 857 (1998).

[5] El-Mabrouk K, Belaiche M, Bousmina M, J. Coll. Interface Sci. 306, 354 (2007).

[6] Nishida K, Ogawa H, Matsuba G, Konishi T, Kanaya T, submitted to J. App. Crys.

[7] El-Mabrouk K, Bousmina M. Rheol. Acta, 45, 877 (2006).

[8] Hashimoto T, Itakura M, Hasegawa H, J. Chem. Phys. 85, 6118 (1986).

[9] Reiter G, Macromolecules, 27, 3046 (1994).

[10] Hashimoto T, Kumaki J, Kawai H, Macromolecules, 16, 641 (1983).

[11] Hashimoto T, Itakura M, Shimidzu N, J. Chem. Phys. 85, 6773 (1986).

[12] Chung H j, Ohno K, Fukuda T, Composto R J, Macromolecules, 40, 384 (2007).

[13] Clarke N, Macromolecules, 38, 6775 (2005).

[14] Cahn J W, Hilliard J E, J. Chem. Phys. 28, 258 (1958).

[15] Cahn J W, J. Chem. Phys. 42, 93 (1965).

[16] Mueller-Buschbaum P, O'Neil S A, Affrossman S, Stamm M, Macromolecules, 31, 5003 (1998).

[17] Chung H, Composto R J, Phys. Rev. Lett. 92, 185704 (2004).

[18] Wang H, Composto R J, Europhys. Lett. 50, 622 (2000).

[19] Wang H, Composto R J, J. Chem. Phys. 113, 10386 (2000). 
[20] Wang H, Composto R J, Interface Science, 11, 237 (2003).

[21] Forrey C, Koberstein J, Pan D H. Interface Science, 11, 211 (2003).

[22] Kanaya T, Ogawa H, Nishida K, Matsuba G. Book of Abstracts, Juelich Soft Matter Days, Bonn, Germany, 14-17 November, 2006..

[23] Liao Y, Su Z, Sun Z, Shi T, An L. Macromol. Rapid Commun. 27, 351 (2006).

[24] Wensink K D F, Je'remoe B. Langmuir, 18, 413 (2002).

[25] Sharma A, Mittal J. Phys. Rev. Lett. 89, 186101 (2002).

[26] Sharma A, Mittal J, Verma R. Langmuir, 18, 10213 (2002) 
[20] Wang H, Composto R J, Interface Science, 11, 237 (2003).

[21] Forrey C, Koberstein J, Pan D H. Interface Science, 11, 211 (2003).

[22] Kanaya T, Ogawa H, Nishida K, Matsuba G. Book of Abstracts, Juelich Soft Matter Days, Bonn, Germany, 14-17 November, 2006.

[23] Liao Y, Su Z, Sun Z, Shi T, An L. Macromol. Rapid Commun. 27, 351 (2006).

[24] Wensink K D F, Je'remoe B. Langmuir, 18, 413 (2002).

[25] Sharma A, Mittal J. Phys. Rev. Lett. 89, 186101 (2002).

[26] Sharma A, Mittal J, Verma R. Langmuir, 18, 10213 (2002) 


\section{Chapter 4}

\section{Phase Separation and Dewetting for Ultra-thin Films in Polystyrene/Poly(vinyl methyl ether) Blend}

\subsection{Introduction}

In Chapter 3, we investigated morphology and kinetics of phase separation as well as dewetting in blend thin films of PS/PVME in two phase region as a function of film thickness in a wide thickness $d$ range from $65 \mu \mathrm{m}$ to $25 \mathrm{~nm}\left(\sim 1.5 R_{\mathrm{g}}\right)$ to elucidate the relation between phase separation and dwetting. However these studies were done for the thickness region above the radius of gyration. For PS and PVME blend ultra-thin films the structure and the morphologies have been studied by two groups [1, 2]. Note that "ultra-thin film" in this chapter means a film with thickness less than twice radius of gyration $2 R_{\mathrm{g}}$ of a polymer chain. It is expected that the blend thin film structure may change near a thickness of $2 R_{\mathrm{g}}$ because polymer chains must be deformed below $2 R_{\mathrm{g}}$. In this chapter, therefore, we have studied the structural and morphology of the PS/PVME blend thin films in a thickness region below $2 R_{\mathrm{g}}$ using light scattering (LS), optical microscope (OM), atomic force microscope (AFM) and scanning near-field optical microscopy (SNOM).

\subsection{Experimental}

Polystyrene (PS) and poly(vinyl methyl ether) (PVME) used in this study have weight-average molecular weights $M_{\mathrm{w}}=280,000$ and 90,000, and the molecular weight distributions in terms of $M_{\mathrm{w}} / M_{\mathrm{n}}=3.01$ and 1.88, respectively, where $M_{\mathrm{n}}$ is number-average molecular weight. Both of PS and PVME were purchased from Scientific Polymer Products, Inc. The detail sample preparation was written in chapter 3 and chapter 4. For the SNOM observation, we used the mixture of PS and perylene-labeled PS (PS-pe) with $M_{\mathrm{w}}=280,000$ and $M_{\mathrm{w}} / M_{\mathrm{n}}=1.67 . \quad$ The fraction of PS, which included PS-pe of $1 \mathrm{wt} \%$ of PS, in the film is $\phi_{\mathrm{PS}}=0.3$. The films were prepared in the same procedure as in chapter 3.

Perylene-labeled polystyrene (PS-Pe) was synthesized by the polymer reaction of PS (Aldrich) and 3-perylenylmethanol, which was synthesized by the reduction of 
3-formylperylene following the Vilsmeiyer reaction of perylene [3]. PS and 3-perylenylmethanol were dissolved in dichloromethane and trifluoroacetic acid and trifluoromethanesulfonic acid were added into the solution. The mixture was stirred for 5 hours at room temperature and then poured into methanol. The obtained polymer was purified by reprecipitation from THF to methanol three times. The molecular weight of the synthesized PS-Pe was determined by gel permeation chromatography (Hitachi, D-7000G) with THF as the eluent and PS (TOSOH) as the standard. The molecular weight was the same as PS before reaction. The perylene fraction of the PS-Pe was determined from UV-Vis absorption spectra by assuming PS-Pe and perylene have the same extinction coefficient. The molar fraction of perylene introduced in the PS chain was found at 0.046 .

Polymer film thicknesses were controlled by varying the polymer concentration in solution. The films were then annealed at $60{ }^{\circ} \mathrm{C}$ for $24 \mathrm{~h}$ after drying in vacuum at room temperature for $24 \mathrm{~h}$. AFM, SNOM measurements were performed at room temperature after quenching the sample from two phase region $\left(=115^{\circ} \mathrm{C}\right)$.

SNOM imaging was performed using a commercial $\alpha$-SNOM (WITec) with a 441 nm laser (BCL-015-440, CrystaLaser) as the excitation source. A cantilever probe was used to scan the sample surface in contacting mode. The probe has a nanoaperture ( $\sim 60 \mathrm{~nm})$ at the tip end, and the light passing through the aperture excites the perylene chromophores inside the polymer film. The signal light from the specimen was collected by a microscope objective (60×, $0.8 \mathrm{NA}$, Nikon) and split by a beam sampler. $4 \%$ of the collected signal was directed to an analog PMT (H5784, Hamamatsu Photonics) to obtain the transmission image, and the rest was detected by a photon counting PMT (H8631, Hamamatsu Photonics) after passing through a long-pass filter (LP02-442RS-25, Semrock) to acquire the fluorescence image. All images were recorded at a pixel size of $3.9 \mathrm{~nm} \times 3.9 \mathrm{~nm}$ and a pixel dwell time of $3.9 \mathrm{~ms}$. With our experimental setup, the surface topography (TP), fluorescence (FL), and transmission (TRANS) images can be simultaneously obtained from the scanning area.

\subsection{Results and Discussion}

First we have performed OM measurements on the PS/PVME blend thin films above and below $2 R_{\mathrm{g}}(\sim 16.6 \mathrm{~nm})$ after the temperature jump from the one phase region to $115^{\circ} \mathrm{C}$ in the two phase region. The time evolution of the $\mathrm{OM}$ images are shown in 
Figure 4.1 for the $42,17,13,8.5 \mathrm{~nm}$ films. As shown in Chapter 3, in the case of rather thick films above $2 R_{\mathrm{g}}$ and below $\sim 200 \mathrm{~nm}$ we observed spinodal decomposition (SD)-like patterns after a long incubation period (see Figure 4.1 (a)). Furthermore we will show in Chapter 5 that the dPS/PVME blend films have a tri-layer structure in the one phase region, consisting of the top PVME layer, the middle blend layer and the bottom PVME layer, and the phase separation of the middle blend layer proceeded during the incubation time and trigged the dewetting. On the other hand, the OM images of the $17 \mathrm{~nm}$ film seems different from the $42 \mathrm{~nm}$ film. The phase separation patter appears earlier than the $42 \mathrm{~nm}$ film although the OM image contrast is lower in the $17 \mathrm{~nm}$ film than the $42 \mathrm{~nm}$ film. It implies the dewetting mechanism in the $17 \mathrm{~nm}$ film is different from the $42 \mathrm{~nm}$ film. As for the 13 and $8.5 \mathrm{~nm}$ films we do not see almost anything in the OM images. This may be due to the very low OM contrast and/or the spatial resolution of the OM apparatus.

We carried out time-resolved LS measurements on the PS/PVME blend films above and below $2 R_{\mathrm{g}}$ after the temperature jump to $115^{\circ} \mathrm{C}$ in the two phase region. The LS profiles are shown in Figure 4.2 for the 42, 20, 17, 13, 8.5, $4.3 \mathrm{~nm}$ films as a function of the annealing time. Down to the $17 \mathrm{~nm}$ film we clearly observed the time evolution of scattering profiles. The scattering peak gradually shifts to the low $q$, showing the growth of the dewetting structure with the annealing time. On the other hand, we do not see the scattering peak in the profile of the $13 \mathrm{~nm}$ film in the $q$ range up to $6 \mu \mathrm{m}^{-1}$ while the scattering intensity slightly increases with the annealing time. This shows that the dewetting pattern has rather small structure and the scattering peak $q_{\text {max }}$ is out of the present $q$ range. With further decreasing the film thickness we do not see any changes in the scattering profiles in the 8.5 and $4.3 \mathrm{~nm}$ films. This may be due to the very low scattering contrast in the thin films similar to the OM observation. The low contrast will be demonstrated by the AFM measurements later. Before going to the AFM results we analyze the LS data in detail.

The peak intensity $I_{\max }$ and the scattering peak position $q_{\max }$ are plotted for the 42, 20 and $17 \mathrm{~nm}$ films as a function of the annealing time in Figures 4.3(a) and (b), respectively. Note that the peak intensity $I_{\max }$ is normalized to the film thickness $d$ $\left(I_{\max } / d\right)$. The $42 \mathrm{~nm}$ film $\left(>2 R_{\mathrm{g}}\right)$ shows a long incubation time and $I_{\max }$ begins to increase at around $30 \mathrm{~min}$. On the other hand the peak intensity $I_{\max }$ gradually increases with annealing time from the beginning of the annealing for the 20 and $17 \mathrm{~nm}$ 


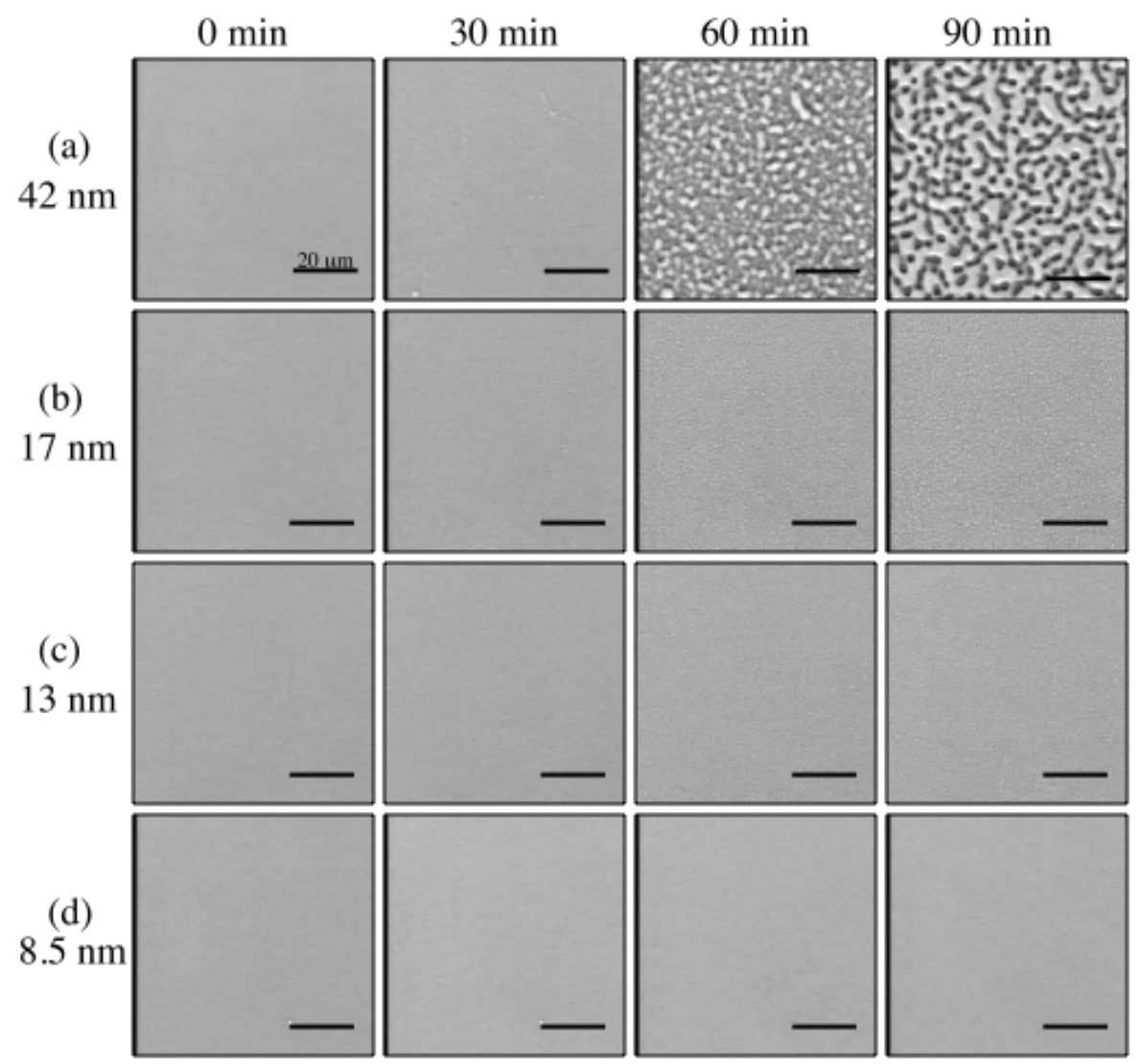

Figure 4.1: Time evolution of OM images of PS/PVME blend thin films after temperature jump to $115^{\circ} \mathrm{C}$ in the two phase region for (a) 42, (b) 17, (c) 13 and (d) $8.5 \mathrm{~nm}$ films. All scale bars are $20 \mu \mathrm{m}$. 

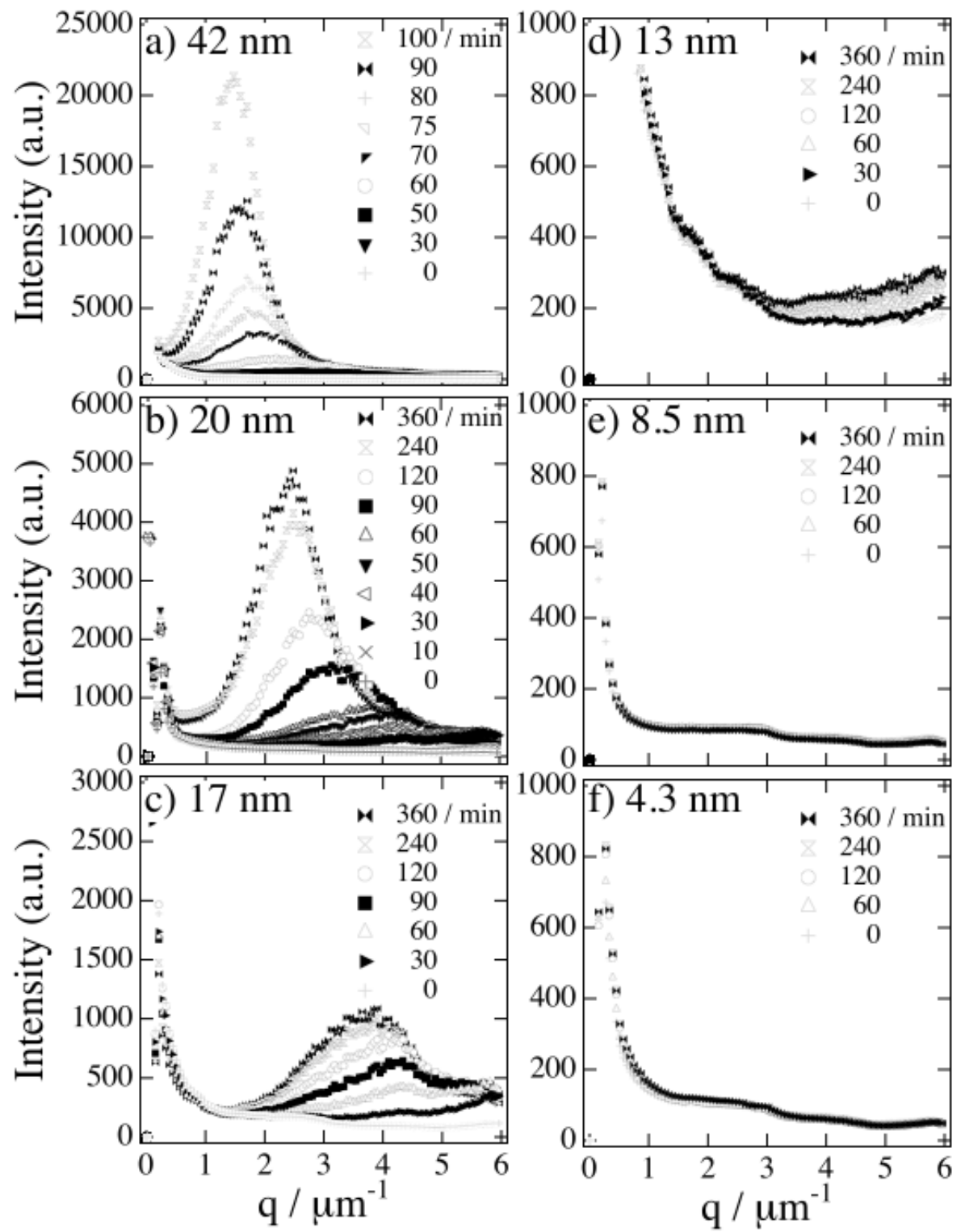

Figure 4.2: Time evolution of LS profiles of PS/PVME blend thin films after temperature jump to $115^{\circ} \mathrm{C}$ in the two phase region for $42,20,17,13$, 8.5 and $4.3 \mathrm{~nm}$ films. 

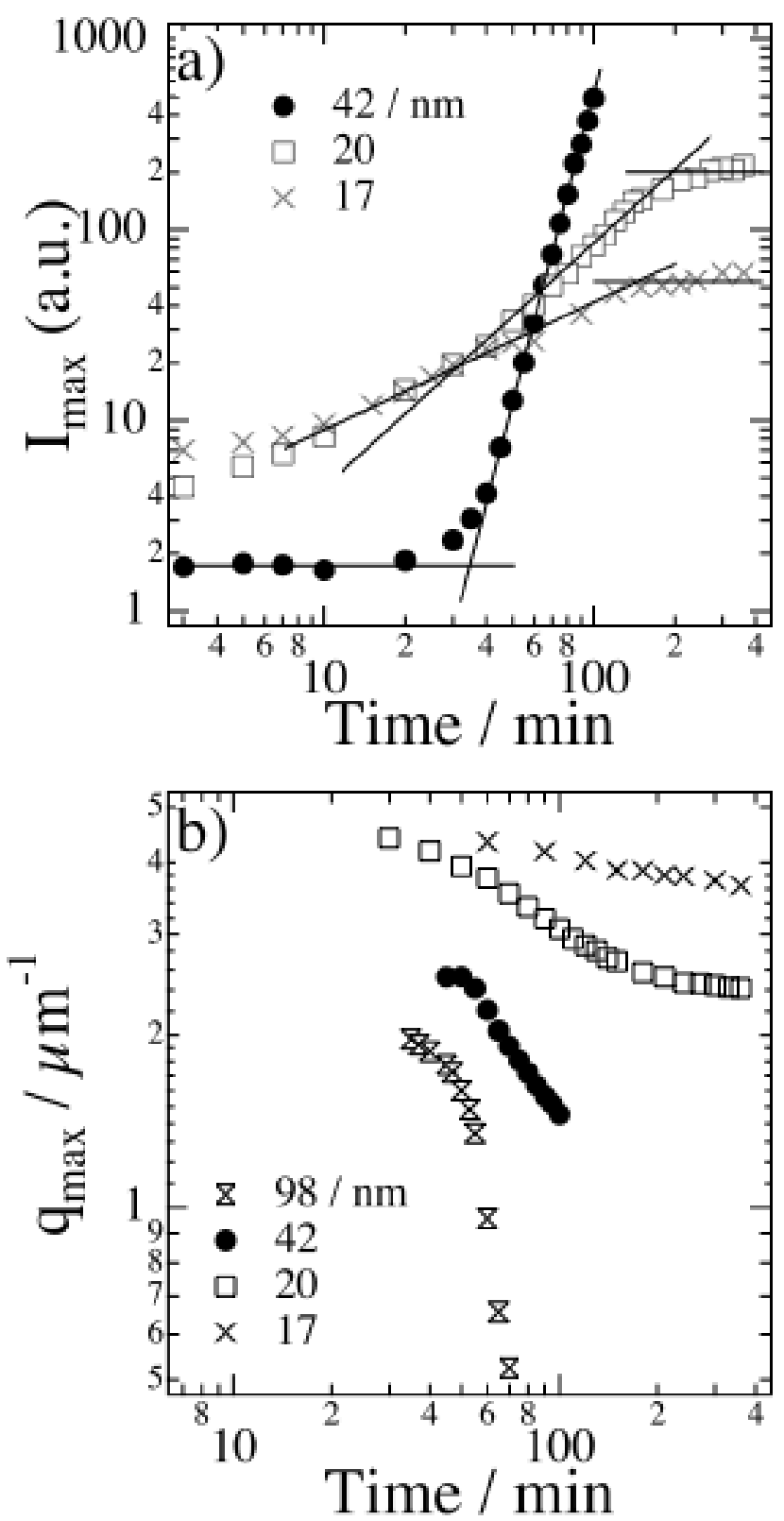

Figure 4.3: Time evolution of the peak intensity $I_{\max }$ and the peak position $q_{\max }$ after temperature jump to $115^{\circ} \mathrm{C}$ in the two phase region for 42, 20 and $17 \mathrm{~nm}$. 
films $\left(\sim 2 R_{\mathrm{g}}\right.$ ), and levels off at $\sim 180$ and $\sim 20 \mathrm{~min}$, respectively. Time evolution patterns are different between the $42 \mathrm{~nm}$ film and the 20 and $17 \mathrm{~nm}$ films, implying that the dewetting mechanism are different between them. The time evolution of $q_{\max }$ is also different between the $42 \mathrm{~nm}$ film and the 20 and $17 \mathrm{~nm}$ films. The peak position $q_{\max }$ rapidly decreases with the annealing time for the $42 \mathrm{~nm}$ film while it levels off after small decrease in $q_{\max }$ for the 20 and $17 \mathrm{~nm}$ films, supporting the change of dewetting mechanism at around $2 R_{\mathrm{g}}$.

In the OM and LS measurements we do not observe any changes in the films below $13 \mathrm{~nm}$ with the annealing, but this must be due to the very low contrast in OM and LS measurements. We expect that something happens in the ultra-thin films below $13 \mathrm{~nm}$. In order to confirm it we performed the AFM measurements on the films below and above $2 R_{\mathrm{g}}$ after annealing at $115^{\circ} \mathrm{C}$ for 0 ad $360 \mathrm{~min}$. The AFM images are shown in Figure 4.4. It is very clear that dewetting occurs in the films below $13 \mathrm{~nm}$, confirming that no changes in the OM and LS patterns are due to the low contrast. The observed AFM images are very interesting. In the case of the films above about $2 R_{\mathrm{g}}(\sim 17 \mathrm{~nm})$ the AFM images show no structure at $t=0 \mathrm{~min}$, and the dewetting patterns are clearly observed at $360 \mathrm{~min}$. On the other hand, the dewetting patterns are already formed at $t=0 \mathrm{~min}$ before annealing for 13, 8.5 and $4.3 \mathrm{~nm}$ films. More interesting is that the 13 $\mathrm{nm}$ film has small holes on the surface (black spots) at $t=0$ min but the droplets of polymers (white part) are observed at $360 \mathrm{~min}$ after annealing, while no structure developments were observed in the dewetting pattern during the annealing for the 8.5 and $4.3 \mathrm{~nm}$ films. Here it should be emphasized that the dewetting pattern is formed even in the one phase region for films below $\sim 2 R_{\mathrm{g}}$ and the dewetting may occur during the film formation process in the spin-coating.

As seen above no time evolution of the dewetting pattern was observed for the thin films below $\sim 10 \mathrm{~nm}$, and hence the time evolution measurements have no meaning in these thin films. However above $2 R_{\mathrm{g}}$, we see the structure developments in the LS measurements for the films above $\sim 17 \mathrm{~nm}$. In order to confirm the structure developments we did the AFM measurements on quenched $17 \mathrm{~nm}$ films after annealing at $115{ }^{\circ} \mathrm{C}$ for a given period. The time evolution of the AFM images are shown in Figure 4.5. We can clearly observe the growth of the pattern. In the early stage the pattern looks like a bi-continuous structure, suggesting the spinodal decomposition like dewetting mechanism. The bi-continuous structure are merged into droplets with 

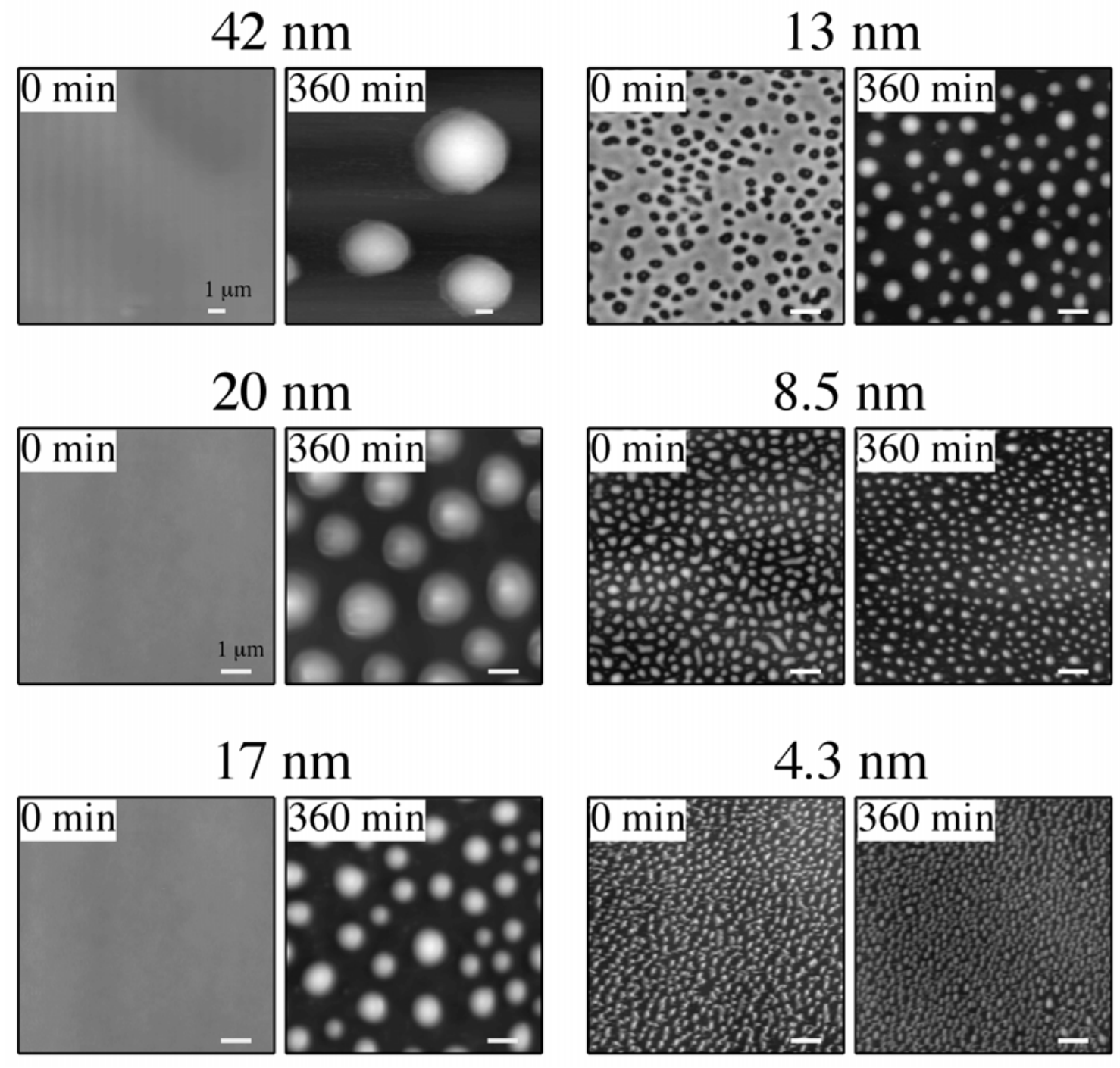

Figure 4.4: AFM images of PS/PVME thin films before annealing $(t=0)$ and after annealing at $115^{\circ} \mathrm{C}$ for $360 \mathrm{~min}$. Scale bar represents $1 \mu \mathrm{m}$. 

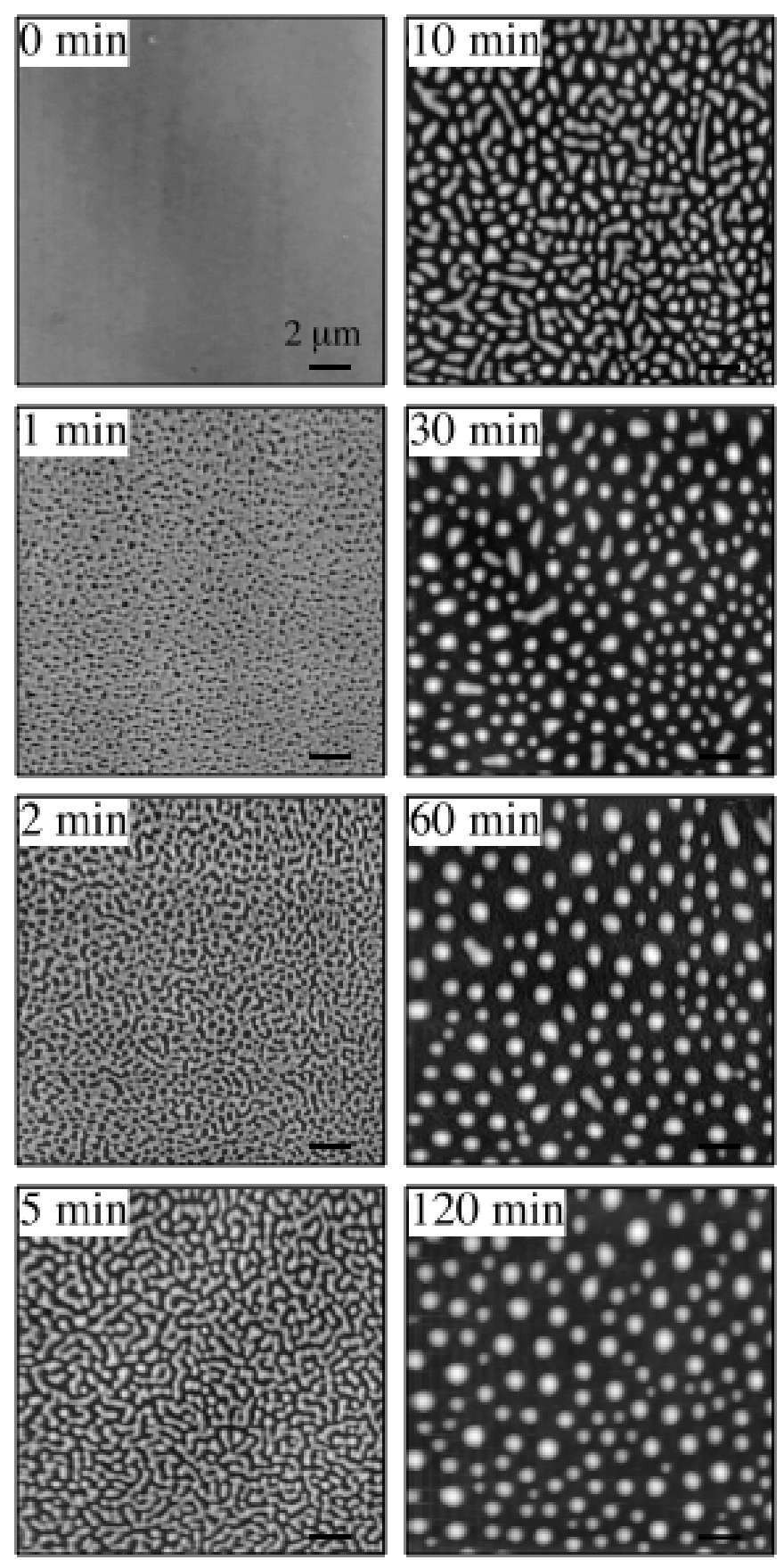

Figure 4.5: Time evolution of AFM images of PS/PVME blend thin films after temperature jump to $115^{\circ} \mathrm{C}$ in the two phase region for the $17 \mathrm{~nm}$ film.

Scale bar represents $2 \mu \mathrm{m}$. 
annealing in the late stage and the structure development is almost finished at 120 min. This agrees with the time evolution of $q_{\max }$ and $I_{\max }$ in the LS profiles (see Figure 4.2).

As was seen in the AFM images the droplet size and the distance between the droplets, which corresponds to a correlation length, depend on the initial film thickness. The distance of the droplets or the correlation length can be evaluated from the peak position $q_{\max }$ through $\lambda_{\max }=2 \pi / q_{\max }$. The peak position $q_{\max }$ was evaluated directly from the LS data (see Figure 4.2) and by performing fast Fourier transform (FFT) of the AFM data in Figure 4.4. We plotted $q_{\max }$ at $t=0$ and $360 \mathrm{~min}$ in Figure 4.6 as a function of the initial film thickness. Above about $2 R_{\mathrm{g}}$ the dewetting dose not occur at $t=0$ just after the film formation, and hence there are no $q_{\max }$ data at $t=0$ above $\sim 17$ nm. The value of $q_{\max }$ from the LS data agrees well with that from the AFM, suggesting that the LS and AFM see the same structure in the measurements. The dewetting pattern is observed at $t=0$ for the films below $\sim 13 \mathrm{~nm}$, and there are $q_{\max }$ at both 0 and $360 \mathrm{~min}$. In the case of the $13 \mathrm{~nm}$ film the shift of $q_{\max }$ to lower value is observed, showing the structure development of the dewetted droplets, while the films less than $13 \mathrm{~nm}$ shows no changes in $q_{\max }$. The initial structure of the droplet just after film formation dose not change any longer with annealing for the films. It is clear that the distance of the coroplets or the correlation length of the droplets depends on the initial thickness of the film. However, we have no plausible explanation on the dependence. Until now we have studied dewetting structure and morphology. However we have to remember that the dewetting occurs in the two phase region. Therefore we performed the SNOM measurements on the dewetted structure to see if the phase separation occurs inside the droplets or not. In the present SNOM measurements PS molecules are labeled by perylene dye molecules, so that we see PS selectively in the droplets. The observed SNOM images are shown in Figure 4.7 for the $17 \mathrm{~nm}$ film before annealing and after annealing for $360 \mathrm{~min}$. In the initial structure before annealing the PS molecules are distributed homogeneously in the in-plane direction of the film. On the other hand, it is clear that PS molecules are located in the droplets after annealing at $115{ }^{\circ} \mathrm{C}$ for $360 \mathrm{~min}$. In order to confirm if PS or PVME exists in the dark part (it terms the sea part hereafter) in the film a part of the film was removed by scratching using a tweezer as shown in Figure 4.8. The fluorescence intensities from the scratched part and the sea part in the non-scratched 
film were compared. It was found that the intensities were almost in the same level, showing no PS molecules in the dark part (the sea part) in the

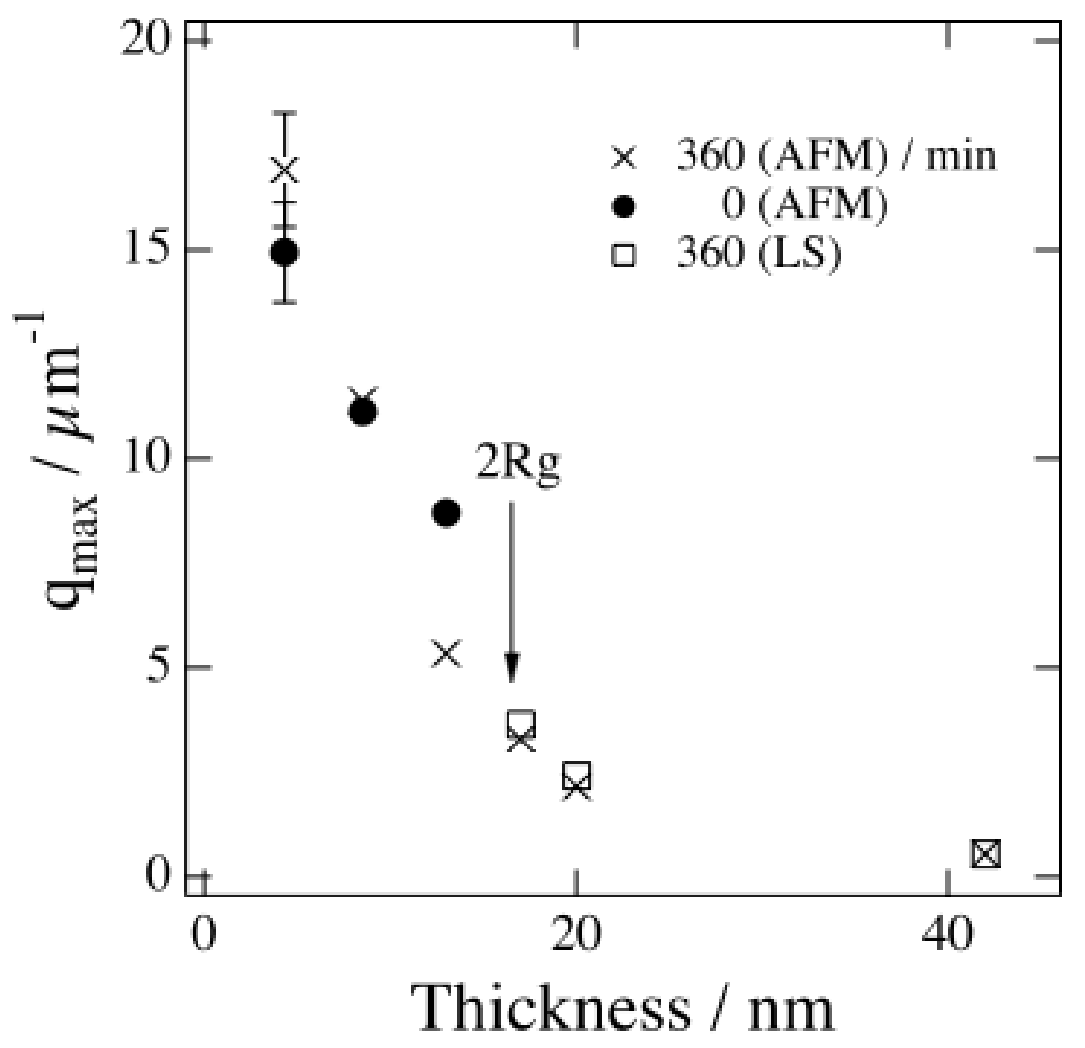

Figure 4.6: Peak position $q_{\max }$ before annealing $(\mathrm{t}=0)$ and after annealing at $115^{\circ} \mathrm{C}$ for $360 \mathrm{~min}$ as a function of initial film thickness. $q_{\max }$ was evaluated directly from the LS data and obtained by FFT of the AFM images. 

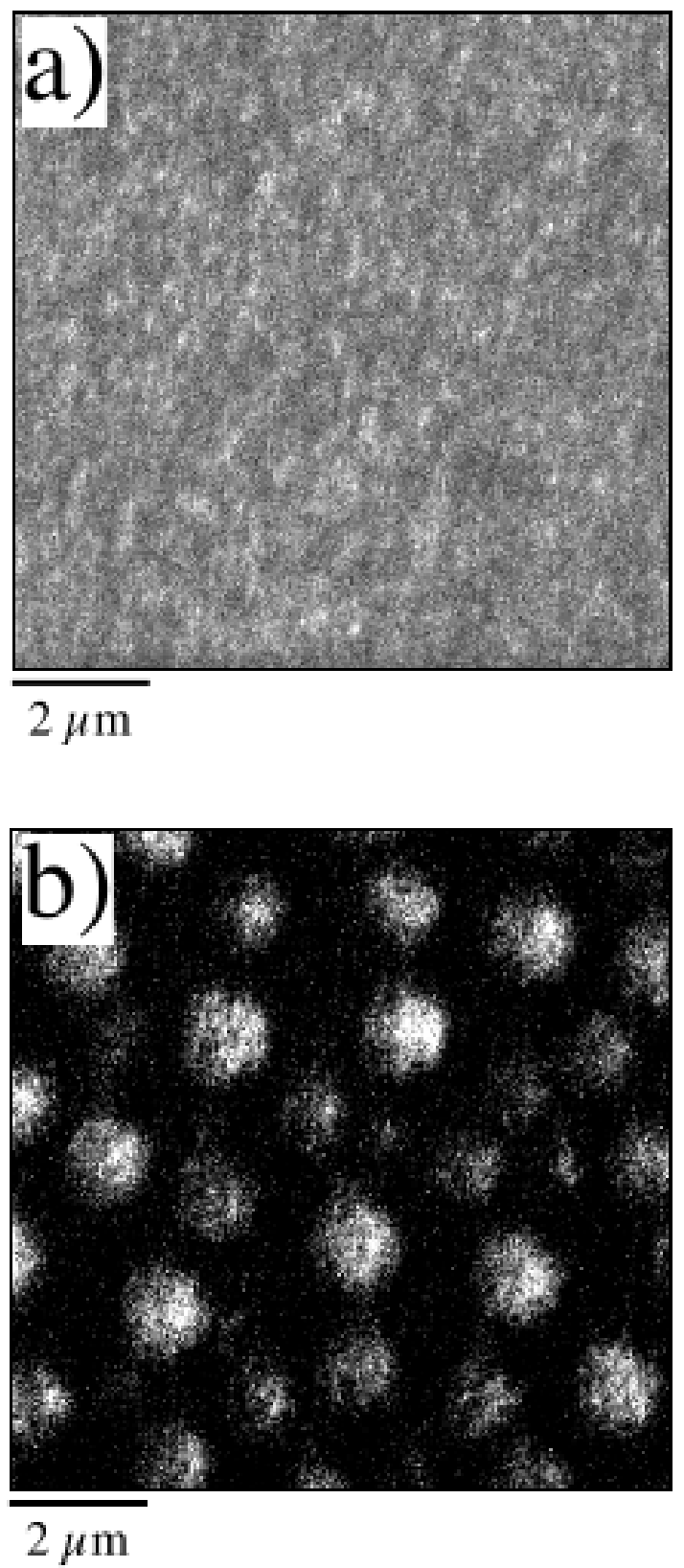

Figure 4.7: SNOM images of a $17 \mathrm{~nm}$ PS/PVME blend thin film before annealing (a) and after annealing at $115{ }^{\circ} \mathrm{C}$ for $360 \mathrm{~min}$ (b). Scale bar represents $2 \mu \mathrm{m}$. 

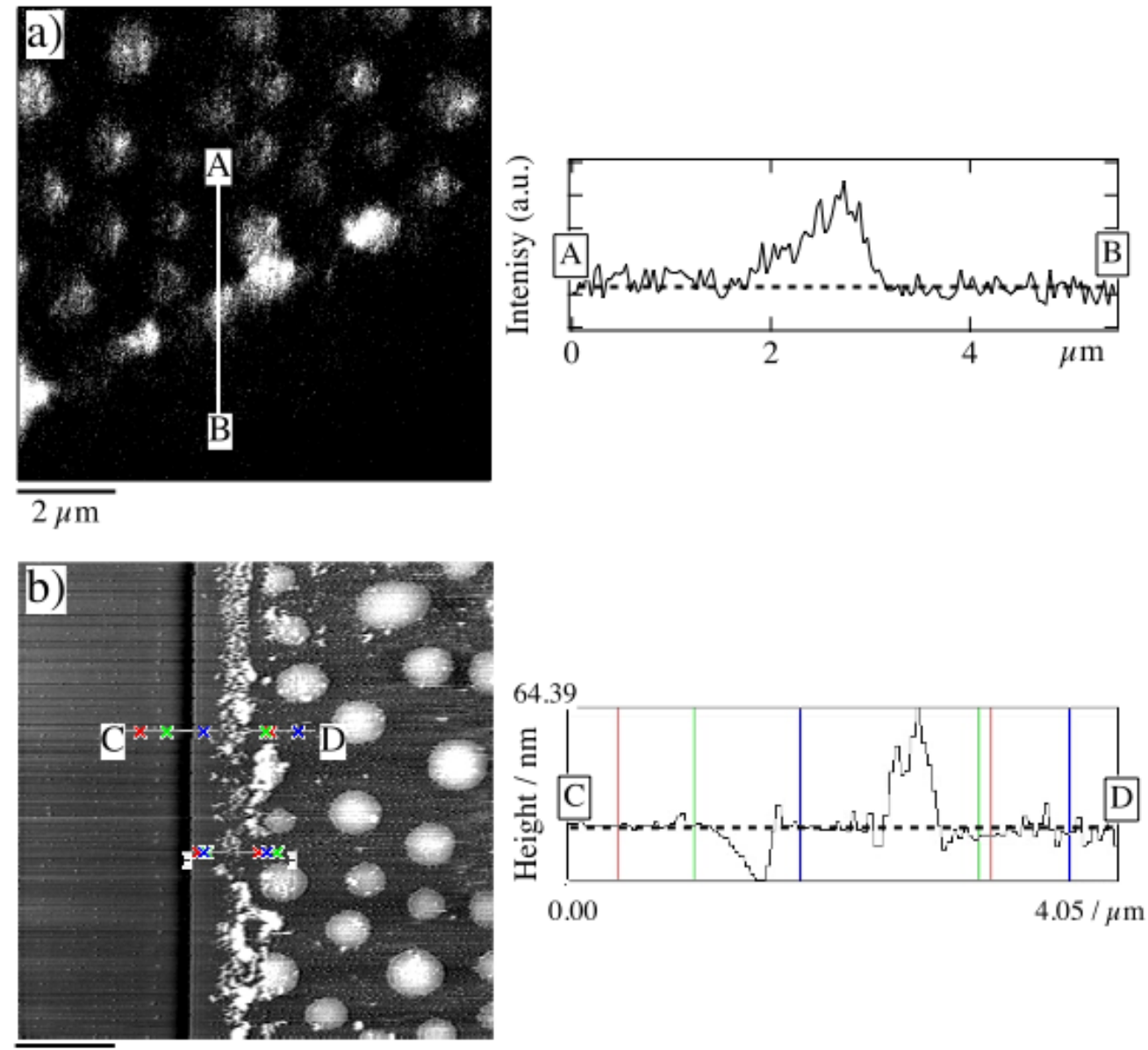

Figure 4.8: SNOM (a) and AFM (b) image of PS/PVME thin films after annealing at $115^{\circ} \mathrm{C}$ for $360 \mathrm{~min}$. Scale bar represents $2 \mu \mathrm{m}$. A part of the film (left part) was removed by scratching using tweezer. (a): Fluorescence intensity along a line A-B. (b) height along a line C-D. 
film. We also measured the height of the scratched part and the sea part along the line (C to D) indicated in Figure 4.8 and the result is shown in Figure 4.8(b). The height is almost identical between the two parts within the height resolution of the AFM apparatus ( $\sim 5 \mathrm{~nm})$. Then we can confirm that neither PVME nor PS molecules are in the sea part of the film, meaning that the dark part is naked surface of the glass substrate.

We also evaluated the average diameters of the droplets in the AFM and SNOM images. We found both of the average diameters were $\sim 1400 \mathrm{~nm}$, which were identical within the experimental error. Taking the resolution functions of the AFM and SNOM it is considered that the thickness of the outer PVME layer in the deroplet is in the order of $\sim 50 \mathrm{~nm}$. The height profile of the droplets was examined based on the AFM data. The average height of the droplets are $\sim 50 \mathrm{~nm}$.

In this study we found that a uniform thin film is not formed on the substrate but the dewetted droplet structure appears in PS/PVME thin films below $\sim 2 R_{\mathrm{g}}$ when a film is prepared by spin-coating from a solution. In the following we will discuss how the dewetted droplet structure is formed on the substrate. The dewetted droplet structure is observed just after the spin-coating the solution, and hence it is hard to imagine the thin film dewets after formation of a uniform film. The dewetted droplet structure may be formed during the spin-coating process. In the solution during the spin-coating process PS and PVME molecules are located near the glass substrate and the air side due to the preferential interactions between PVME and air [4] when the solvent is rather evaporated and many polymer chains feel the interactions. In the in-plane direction PS and PVME molecules may be homogeneously distributed. This is schematically shown in Figure 4.9(a). When further evaporation of the solvent molecules proceeds and polymer molecules form a film on the substrate, there are tow possible ways to form a uniform film because interpenetration of polymer chains is impossible in a thin film [5]: one is two layer structure consisting of the top PVME and bottom PS layers and the other is one layer structure of mixture of PVME and PS, which are schematically illustrated in Figure 4.9(b) and (c), respectively. In the former case PS and PVME chains must be deformed very much from the normal coil size to form a ultra-thin uniform film because the total amount of PS and PVME is very small. In the later case, PS and PVME must be mixed in a one layer against the preferential interactions between PVME and air. Free energies in these uniform films must be 


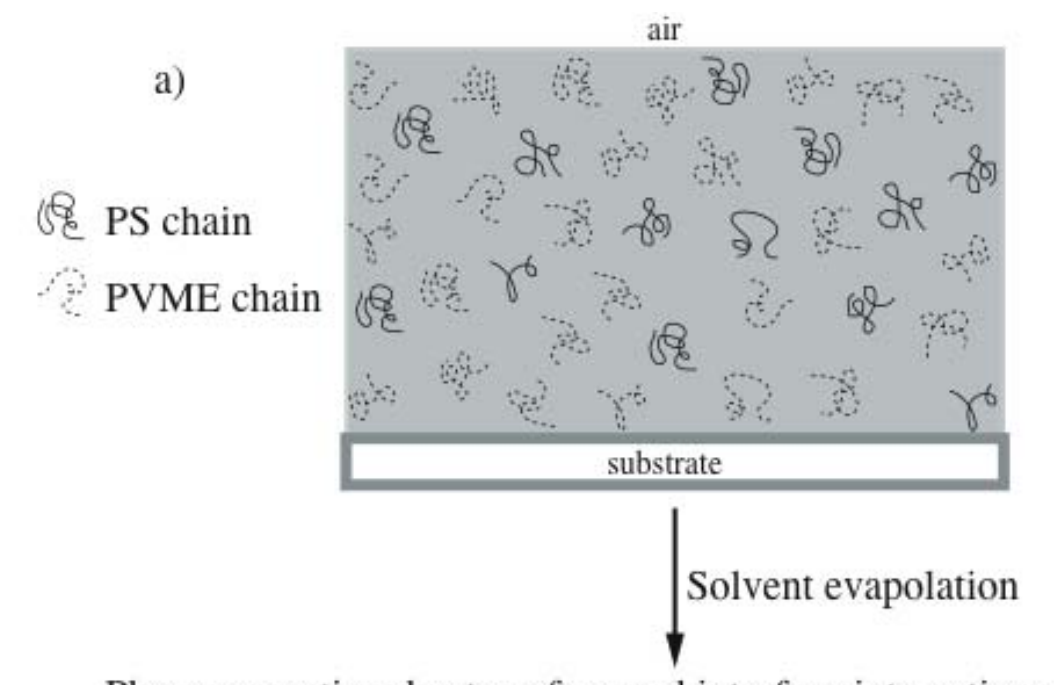

Phase separation due to suface and interface interactions in solution
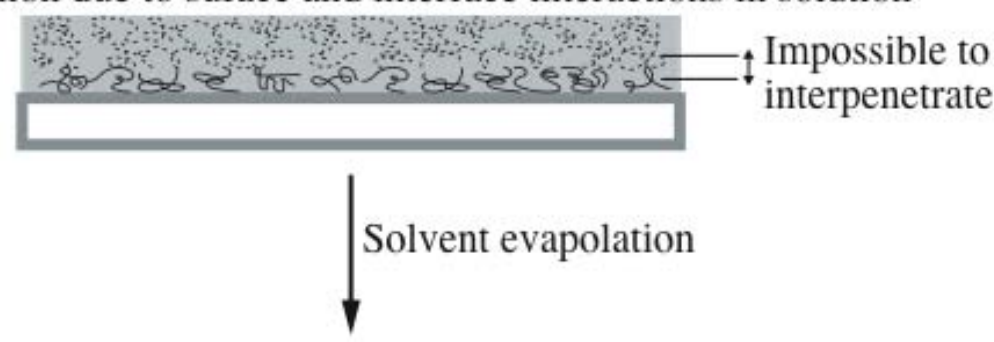

b) two layer structure of PS and PVME c) one layer of mixture of PS and PVME
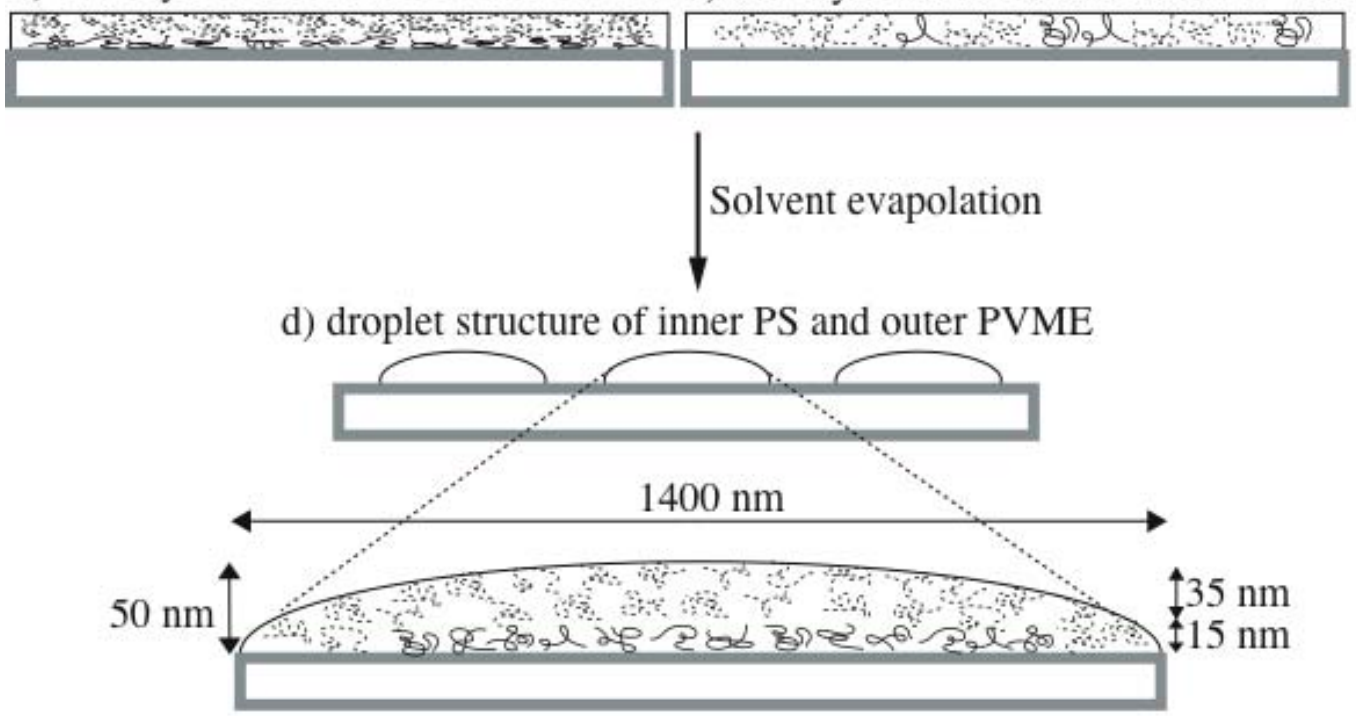

Figure 4.9: Schematic sketches of film formation process during the spin-coating process for a thin film below $\sim 2 R_{g}$. 
very high, and in order to reduce the free energy the polymer chains form a droplet consisting of outer PVME layer and inner PS droplet as shown in Figure 4.9(d).

\subsection{Conclusion}

In this chapter we studied the structure and morphology of the PS/PVME blend thin films below about twice radius of gyration. The OM and LS studies have shown that the dewetting mechanism changed when the film thickness approached twice radius of gyration $2 R_{\mathrm{g}}$ of polymer chains although the measurements were impossible for the thin films below about $13 \mathrm{~nm}$ due to the low contrast and/or the spatial resolution problems. We therefore did the AFM measurements on the PS/PVME blend thin films below and above $2 R_{\mathrm{g}}$ before annealing and after annealing at $115{ }^{\circ} \mathrm{C}$ in the two phase region for $360 \mathrm{~min}$. It was found that the dewetting occurred to form droplet structure in the thickness region below $\sim 13 \mathrm{~nm}$ when the film was prepared by spin-coating the solution even in the one phase region. The SNOM measurements were also done on the dewetted structure to see whether or not the phase separation occurs inside the droplets using perylene labeled PS. It was found that PS was inside the dewetted droplet. Comparing the SNOM and the AFM data it was found that the outer PVME layer was in the order of $\sim 50 \mathrm{~nm}$. Finally we discussed how the dewetted droplet structure was formed during the spin-coating process of the solution. 


\section{References}

[1] Karim A, Slawecki T M, Kumar S K, Douglas J F, Satija S K, Han C C, Russell T P, Liu Y, Overnay R, Sokolov J, Rafailovich M H. Macromolecules, 31, 857 (1998).

[2] Tanaka K, Yoon J S, Takahara A, Kajiyama T. Macromolecules, 28, 934 (1995).

[3] Aoki H, Ito S. J. Phys. Chem. B. 105, 4558 (2001).

[4] Forrey C, Koberstein J, Pan D H, Interface Science, 11, 211 (2003).

[5] de Gennes P G, 'Scaling Concepts in Polymer Physics', Cornell University Press, Ithaca (1979). 


\section{Chapter 5}

\section{Composition Fluctuations before Dewetting in Polystyrene/Poly(vinyl methyl ether) Blend Thin Films}

\subsection{Introduction}

In chapter 3, we investigated morphology and kinetics of phase separation as well as dewetting in blend thin films of PS/PVME on glass substrate in two phase region as a function of film thickness in a wide thickness $d$ range from $65 \mu \mathrm{m}$ to $42 \mathrm{~nm}\left(\sim 2.5 R_{\mathrm{g}}\right)$ to elucidate the relation between phase separation and dewetting. We found that the dewetting occurred in a spinodal decomposition (SD) mechanism after a long incubation time in a thickness range below $\sim 200 \mathrm{~nm}$. The observations indicated a layered structure was formed in the incubation time before the dewetting although direct evidences for the layered structure were not obtained in chapter 3 . In order to confirm the prediction of the layered structure we have mainly studied structural development in the depth direction during the incubation period before the dewetting for thin blend films of deuterated PS (dPS) ad PVME in a thickness range below 200 nm using neutron reflectivity.

\subsection{Experimental}

Polystyrene (PS) and poly(vinyl methyl ether) (PVME) used in this study have weight-average molecular weights $M_{\mathrm{w}}=280,000$ and 90,000, and the molecular weight distributions in terms of $M_{\mathrm{w}} / M_{\mathrm{n}}=3.01$ and 1.88, respectively, where $M_{\mathrm{n}}$ is number-average molecular weight. We also used deuterated polystyrene (dPS) with weight-average molecular weight $M_{\mathrm{W}}=280,000$ and the molecular weight distribution $M_{\mathrm{w}} / M_{\mathrm{n}}=1.08$ for neutron reflectivity measurements. Both of PS (dPS) and PVME were purchased from Scientific Polymer Products, Inc. The detail sample preparation was written in the former chapter. Blend films of dPS and PVME were prepared on a cleaned Si wafer with native oxide layer in the same manner as for the PS and PVME films. The critical weight fraction of PS is 0.3 and the critical temperature is $104.5^{\circ} \mathrm{C}$. All the measurements were done on the blend samples with the critical concentration. Light scattering (LS) and Optical microscope (OM) measurements were done on the 
film samples after temperature jump to the two phase region at $115{ }^{\circ} \mathrm{C}$ from the one phase region annealed at $90{ }^{\circ} \mathrm{C}$ for $30 \mathrm{~min}$. Atomic force microscope (AFM) measurements were performed at room temperature after quenching the sample from two phase region $\left(=115^{\circ} \mathrm{C}\right)$ to examine the surface morphology of the films.

Neutron reflectivity measurements were done on MINE-II reflectometer at JRR-3 reactor in Tokai [1]. Incident neutron wavelength was $0.88 \mathrm{~nm}$ and the $q$ range covered in the present measurements was 0.07 to $1.0 \mathrm{~nm}^{-1}$. The measurements was conducted at room temperature after quenching the sample from two phase region $\left(=115^{\circ} \mathrm{C}\right)$. We analyzed the observed reflectivity data using the Paratt32 program in Hahn-Meitner-Institute [2], which is based on a recursion formula derived by Parratt [3] to calculate reflectivity from successive interfaces and modified to include effects of interfacial roughness.

\subsection{Results and Discussion}

We first recall the previous results in Chapter 3 to clarify the aim of this work. We have studied the phase separation and dewetting processes of blend thin films of PS and PVME with the critical concentration in a wide thickness range of $42 \mathrm{~nm}$ to $65 \mu \mathrm{m}$ after a temperature jump to $115^{\circ} \mathrm{C}$ in the two phase region from the one phase region using OM, AFM and LS. We found that both of the phase separation and dewetting processes depended on the film thickness, and were classified into four thickness regions. In order to explain the characteristic features in the four regions the typical $\mathrm{OM}$ and AFM images at 90 min after the temperature jump are shown in Figures 5.1(a) and (b), respectively, and the time evolutions of the LS profiles are shown in Figure 5.1(c) for the four regions. In the first region above $\sim 15 \mu \mathrm{m}$ (Region I), the spinodal decomposition (SD) type phase separation is observed in the OM image, which is also confirmed from the time evolution of LS intensity. In this region no dewetting is observed in the AFM image, and hence this region can be regarded as bulk region. In the second region between $\sim 15$ and $\sim 1 \mu$ m (Region II) the SD type phase separation proceeds in the early stage similar to Region I while the characteristic wavelength decreases with the film thickness due to the confinement effect of film thickness in this region. In the late stage the dewetting is induced by the phase separation. In the third region between $\sim 1 \mu \mathrm{m}$ and $\sim 200 \mathrm{~nm}$ (Region III) the dewetting is observed even in the early stage. The dewetting morphology is very irregular as seen in Figures 5.1(a) and 
(a)

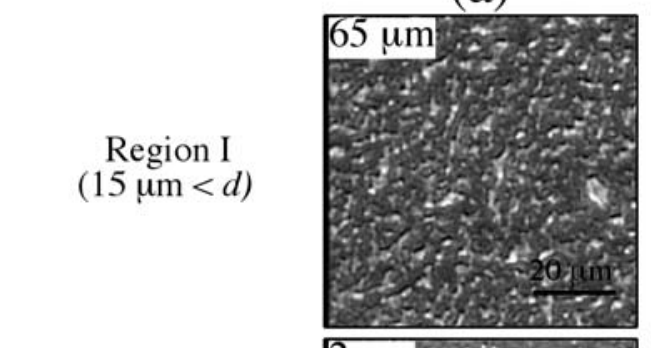

Region II

$(1 \mu \mathrm{m}<d<15 \mu \mathrm{m})$

$(200 \mathrm{~nm}<d<1 \mu \mathrm{m})$

Region IV $(d<200 \mathrm{~nm})$
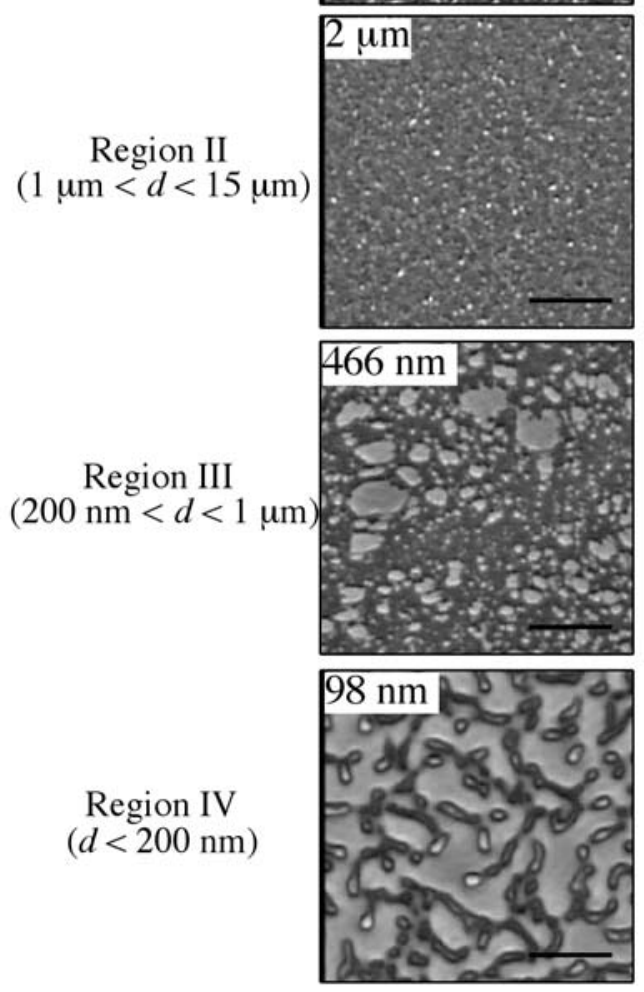

(b)
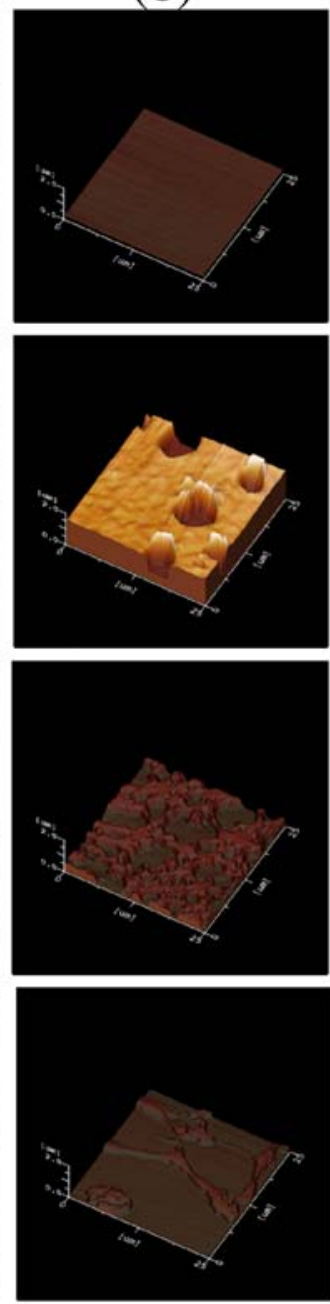

(c)
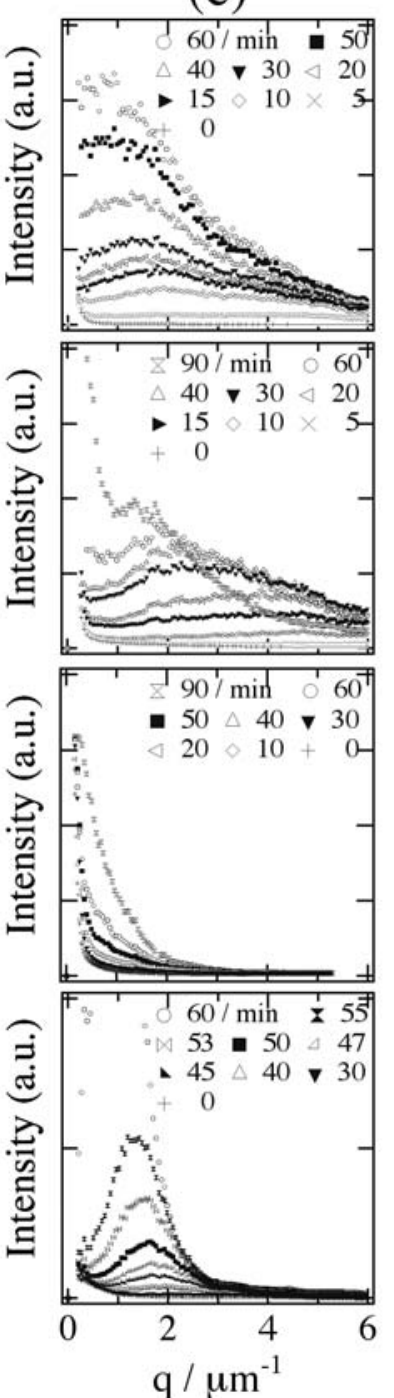

Figure 5.1: OM (a) and AFM (b) images of PS/PVME blend thin films after temperature jump to $115{ }^{\circ} \mathrm{C}$ in the two phase region for four thickness $d$ regions. Region I $(d>15 \mu \mathrm{m}): 65 \mu \mathrm{m}, \quad$ Region II $(1 \mu \mathrm{m}<d<15 \mu \mathrm{m}): 2 \mu \mathrm{m}, \quad$ Region III (200 nm $<d<1 \mu \mathrm{m}): 466 \mathrm{~nm}, \quad$ IV $(: d<200 \mathrm{~nm}): 98 \mathrm{~nm}$ and time evolution of LS profiles (c) after temperature jump to $115^{\circ} \mathrm{C}$ in the two phase region. Scale bars in OM images are $20 \mu \mathrm{m}$. Z-range is $2.0 \mu \mathrm{m}$ and $\mathrm{x}-\mathrm{y}$ size is $25 \mu \mathrm{m} \times 25 \mu \mathrm{m}$ for all AFM images. 
(b) and no definite characteristic wavelength is observed in the LS profile. It is expected that the irregular morphology is induced by mixing up the characteristic wavelengths of the phase separation and the dewetting. In the forth region below $\sim 200$ $\mathrm{nm}$ (Region IV) the dewetting occurs after a long incubation time with a characteristic wavelength, giving a peak in the LS profile as seen in Figure 5.1(c). Note that the LS profiles in Figure 5.1(c) are shown until 60 min for Region IV. Generally speaking, the SD type mechanism occurs in an unstable region [4, 5], meaning that no incubation time is required. However, the long incubation time is observed before the dewetting, suggesting that some structure formation occurs before the onset of the SD type dewetting. Therefore, we examined structure formation in the incubation period before dewetting to discuss the dewetting mechanism in Region IV below $\sim 200 \mathrm{~nm}$.

In Figure 5.2, time evolution of the OM images is shown for the $98 \mathrm{~nm}$ film after temperature jump to $115{ }^{\circ} \mathrm{C}$ to see what happens in the incubation period. Nothing is observed in the OM images before 20 min within the present $\mathrm{OM}$ contrast and the spatial resolution while very weak in-plane fluctuations are recognized after $\sim 20 \mathrm{~min}$ as seen in Figure 5.2. At $\sim 30$ min the SD type patterns becomes rather clear and grows in size with the annealing time. According to the changes in the $\mathrm{OM}$ images, we also observe the changes in the LS profiles. In Figure 5.3 we plot the time evolution of the LS intensity $I_{\max }$ and the peak position $q_{\max }$. The LS peak appears at $30 \mathrm{~min}$ after the temperature jump, corresponding to the appearance of the SD pattern in the OM image. It is noted that the LS intensity slightly increases until $~ 30 \mathrm{~min}$ in the incubation period, which must be due to the very weak in-plane fluctuations observed in the OM image. After 30 min the peak intensity $I_{\max }$ increases rapidly with the annealing time and the peak position shifts to lower $q$. It is clear from the AFM images in Figure 4.1(b) that this SD type pattern is caused by the dewetting.

As discussed in Chapter 3 we expect that some layered structure is formed during the incubation period, and triggers the dewetting through the capillary fluctuations mechanism [6, 7] or the composition fluctuation one [8, 9-12]. However, no direct evidence was provided for the layered structure. In order to confirm the layered structure we examined the composition fluctuations in the direction normal to the surface (the depth direction) before dewetting using neutron reflectivity (NR), which is one of the most powerful tools to study composition and/or density fluctuations in the depth direction. 

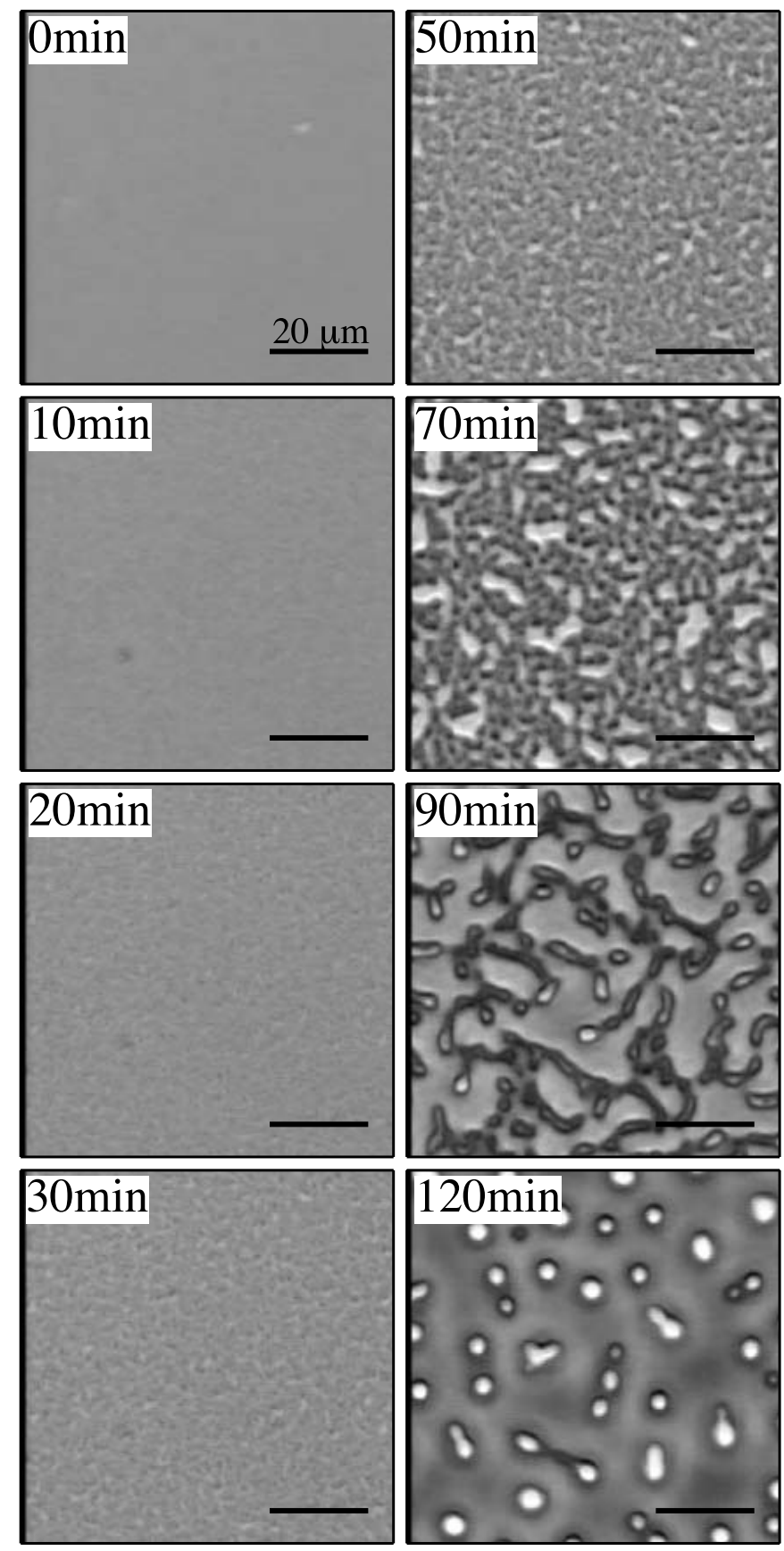

Figure 5.2: Time evolution of OM images of PS/PVME blend film $98 \mathrm{~nm}$ thick after temperature jump to $115{ }^{\circ} \mathrm{C}$ in the two phase region. Scale bars in OM images are $20 \mu \mathrm{m}$. 


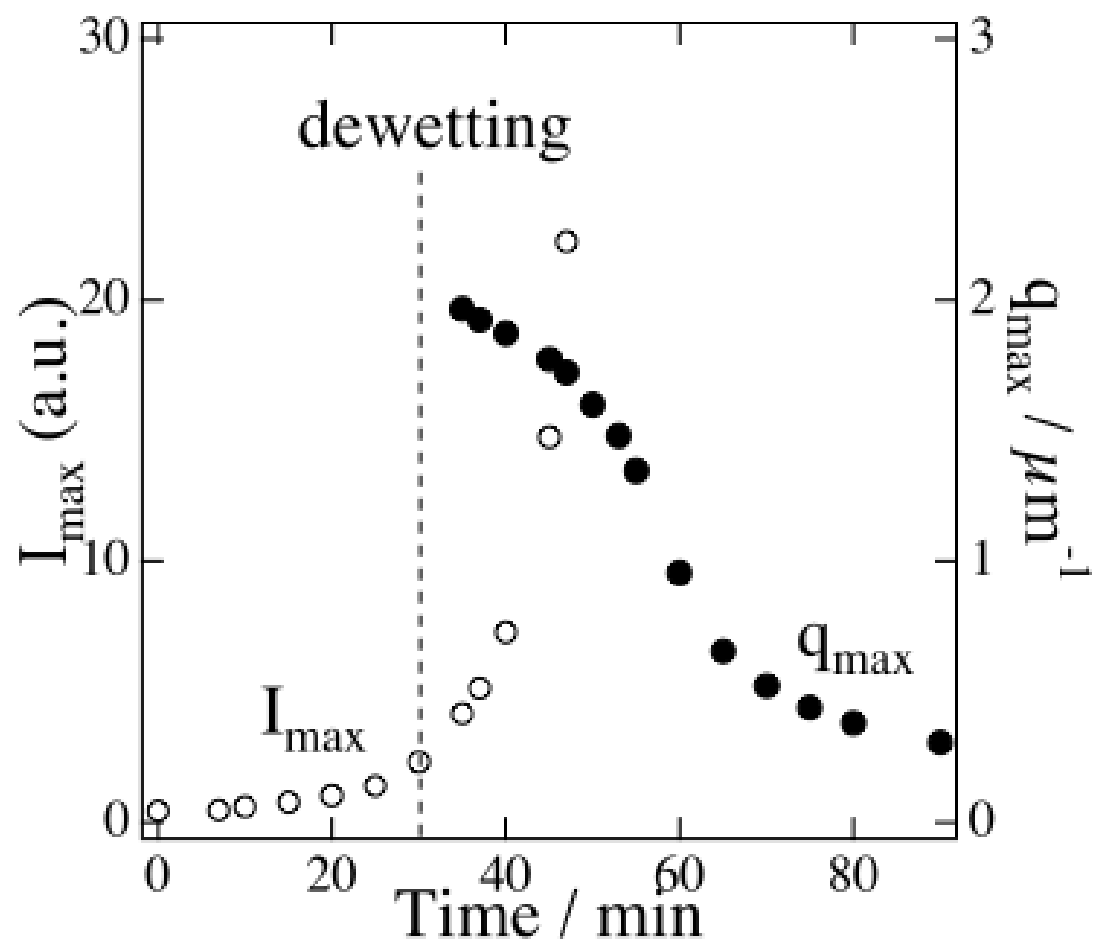

Figure 5.3: Time evolution of peak position $q_{\max }$ and peak intensity $I_{\max }$ in the LS profile of PS/PVME blend film $98 \mathrm{~nm}$ thick after temperature jump to $115{ }^{\circ} \mathrm{C}$ in the two phase region. Peak intensity $I_{\max }$ in the incubation period before $\sim 30 \mathrm{~min}$ is intensity at $q=2 \mu \mathrm{m}^{-1}$. 
We used a Si wafer as a substrate in the NR measurements instead of a glass substrate, which was used for OM, LS and AFM in Chapter 3. It is known that the dewetting is affected by interface interactions between polymer film and substrate. In addition we used deuterated polystyrene (dPS) in the NR experiments. It would be impossible to consider these two systems (PS/PVME on a glass substrate and dPS/PVME on a Si substrate) are identical. Therefore we first examined the dPS/PVME blend film $98 \mathrm{~nm}$ thick using confocal laser scanning microscope (CLSM). We found that the dewetting patterns were almost identical but the onset time was slower in the case of the Si substrate than the glass one. Exactly speaking, it is $60 \mathrm{~min}$ at $115{ }^{\circ} \mathrm{C}$ for the $\mathrm{Si}$ substrate and $30 \mathrm{~min}$ for the glass substrate. No other large differences were observed between the Si and glass substrates. However, the phase separation and dewetting are so sensitive to the substrates that we discuss the results on the dPS/PVME thin films on the Si substrate independent of the PS/PVME thin films on the glass substrate in this paper.

We performed NR measurements on the dPS/PVME blend films at room temperature in the one phase region before annealing at $115{ }^{\circ} \mathrm{C}$ in the two phase region. The observed NR profiles are shown in Figure 5.4 for the 180, 98, 42 and 25 nm films. The well defined fringed patterns were observed in the profiles. The NR profiles were analyzed by fitting a single layer model on a Si substrate with a native oxide layer to the observed data, but good fitness was not obtained. Then, we tried bi-layer and tri-layer models consisting of surface PVME layer at the air, middle blend layer, and interface PVME layer at the Si substrate because the preferential interactions are considered between PVME and air [13-17] and between PVME and Si substrate [18, 19]. In this model the surface roughness $\sigma_{\mathrm{NR} \text {,surf }}$ and the interface roughness $\sigma_{\mathrm{NR} \text {,inter }}$ were introduced by an error function (eq. (1))

$$
\operatorname{erf}(x)=\int_{x}^{\infty} \frac{1}{\sqrt{2 \pi} \sigma_{i}} \exp \left(-\frac{t^{2}}{2 \sigma_{i}^{2}}\right) d t \quad(i=\mathrm{NR}, \text { inter or NR,surf })
$$

It was found that the tri-layer model gave good agreements between the observed reflectivity and the calculated one as shown in Figure 5.4. In inset of Figure 5.4 the scattering length density evaluated in the fit is shown for each film. The estimated surface and interface PVME layers are $\sim 4 \mathrm{~nm}$ and $\sim 5 \mathrm{~nm}$ thick and almost independent 


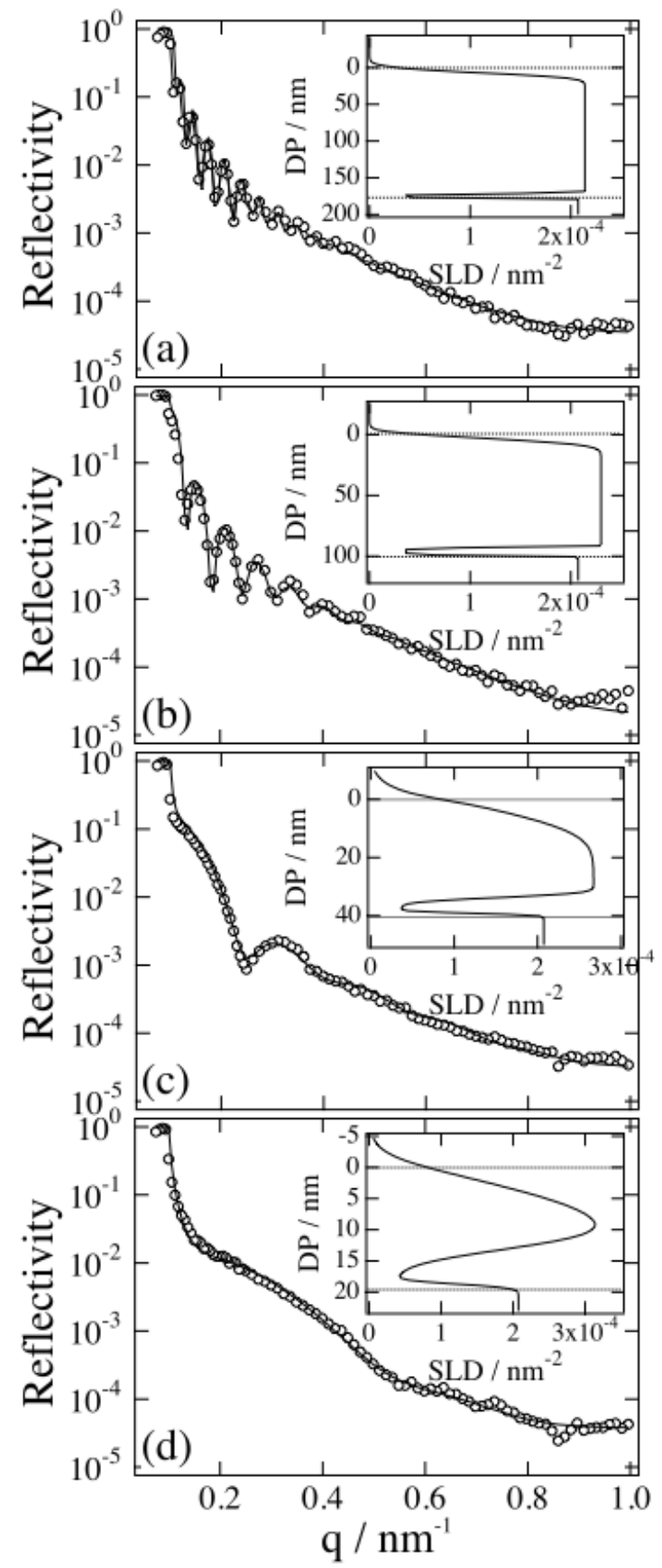

Figure 5.4: NR profiles for dPS/PVME blend films 180, 98, 42 and $25 \mathrm{~nm}$ thick at $25{ }^{\circ} \mathrm{C}$ in the one phase region and the results of fits (solid curves) with the tri-layer model. Insets show scattering length density (SLD) profiles in depth direction evaluated in the NR profile fits. 
of the total film thickness within the experimental error. The surface roughness, the interface roughness between the surface PVME layer and the middle blend layer and the interface roughness between the middle blend layer and the bottom PVME layer are 3.5, 4.5 and $5 \mathrm{~nm}$ for the $180 \mathrm{~nm}$ film, respectively, and slightly decrease with the total film thickness. It is expected that the surface and interface PVME layer formation increase the dPS concentrations in the middle blend layer, especially in the thin films. Hence, we have evaluated the dPS concentrations in the middle blend layer and found that the effect is negligible for the 180 and $98 \mathrm{~nm}$ films, but not negligible for the $42 \mathrm{~nm}$ and 25 $\mathrm{nm}$ films. In case of the $25 \mathrm{~nm}$ film the estimated dPS concentration $\phi_{\mathrm{PS}}$ in the middle blend layer is 0.47. Therefore, the further experiments were performed on the $98 \mathrm{~nm}$ film. In any case the present results clearly show that the dPS/PVME blend thin films on the Si substrate have tri-layer structure even in the one phase region due to the preferential interactions.

In the next step we have examined the time evolution of the tri-layer structure in the $98 \mathrm{~nm}$ film during the incubation period before the dewetting. The film was annealed at $115^{\circ} \mathrm{C}$ in the two phase region for a given period, and quenched to room temperature in the one phase region. The NR measurements were performed on the quenched film. In Figure 5.5(a) the observed NR profiles are shown for various annealing periods. The fringed pattern is gradually smeared with the annealing time even in the incubation period ( $60 \mathrm{~min}$ ), suggesting that the surface roughness and/or the interface roughness increases with the annealing time before the dewetting. At 120 min the fringed pattern is completely smeared out, showing the film is dewetted. It is surprising that the fringed pattern with a long period was again observed at $360 \mathrm{~min}$, implying that a thin layer was formed on the surface of the substrate after the dewetting. This problem will be discussed later.

The NR profile calculated from the tri-layered model was fitted to the observed one to evaluate the thickness and the surface and interfacial roughness for each layer. The results of fits are shown by solid curves in Figure 5.5(a) and the depth profiles of the corresponding scattering length density are plotted in Figure 5.5(b). The interface roughness $\sigma_{\mathrm{NR} \text {,inter }}$ between the top PVME layer and the middle blend layer increases with the annealing time during the induction period, but the interface roughness between the bottom PVME layer and the middle blend layer stays almost constant ( 5 

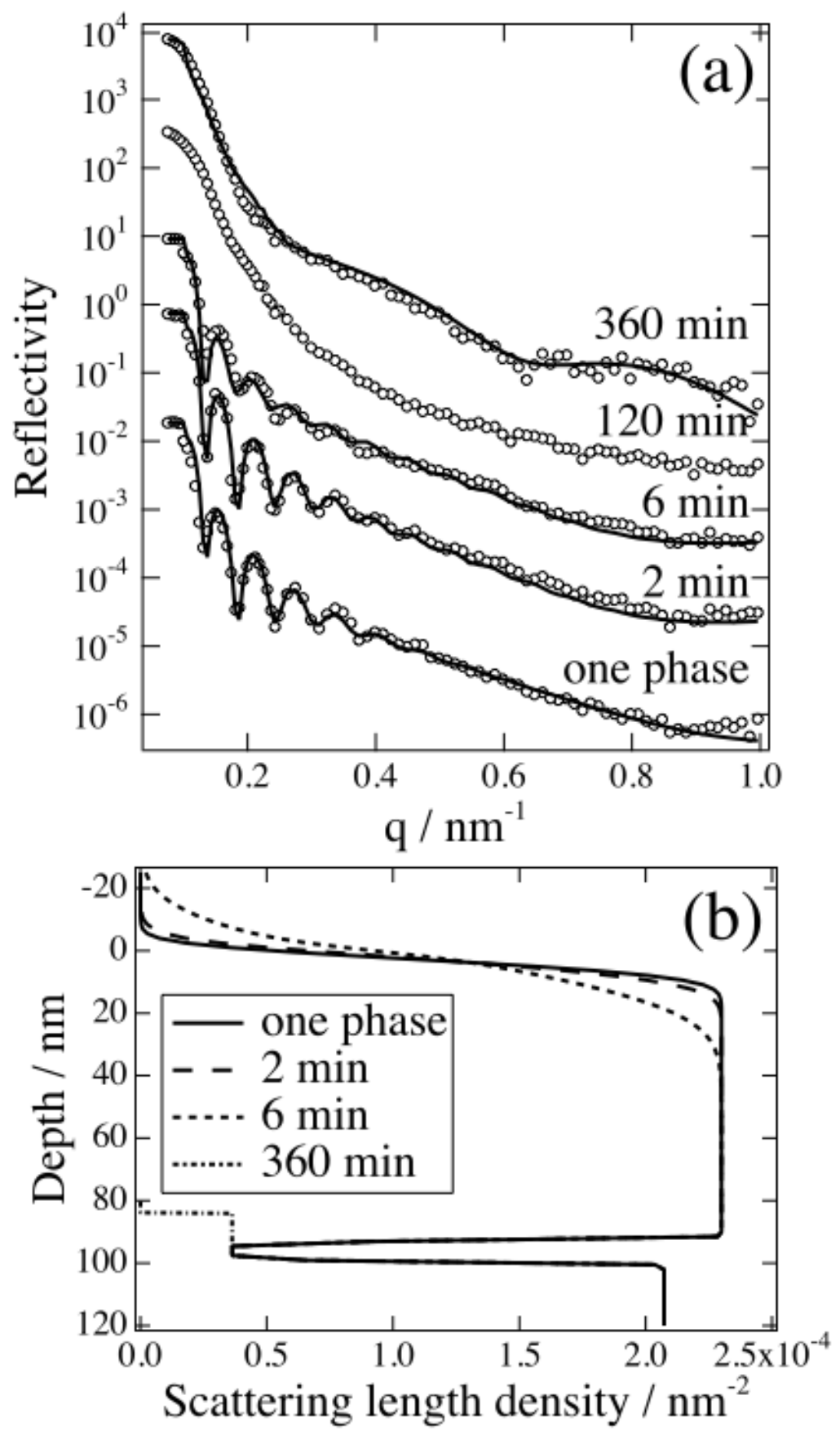

Figure 5.5: (a): Time evolution of NR profile for dPS/PVME blend film $98 \mathrm{~nm}$ thick annealed at $115{ }^{\circ} \mathrm{C}$ in the two phase region. Measurements were done after quenching to room temperature. The solid curves are the results of fits with the tri-layer model. (b): Scattering length density (SLD) profiles in depth direction as a function of annealing time. 
$\mathrm{nm}$ ). The surface roughness $\sigma_{\mathrm{NR} \text {,surf }}$ of the top PVME layer seems to increase with the annealing time, but it is noted that the NR profile is not sensitive to the surface roughness of the top PVME layer because of the low scattering length density of PVME so that we do not discuss the surface roughness here. The former interface roughness $\sigma_{\mathrm{NR} \text {,inter }}$ are plotted as a function of the annealing time in Figure 5.6. It is evident that they increase even during the incubation period. Before going to the discussion on the time evolution of the roughness, we introduce the surface roughness $\sigma_{\mathrm{AFM} \text {,surf }}$ evaluated by the AFM measurements because the surface roughness $\sigma_{\mathrm{NR}, \text { surf }}$ estimated by the NR measurements includes large error.

The observed AFM images of the dPS/PVME film $98 \mathrm{~nm}$ thick are shown in Figure 5.7 for various annealing times in the incubation period. The surface is very smooth at the annealing time $=0 \mathrm{~min}$. As the annealing proceeds no significant changes were observed during the incubation period. In order to evaluate the surface roughness the AFM data was analyzed in the following way. The distribution of surface heights $h$ was calculated from the AFM data. An example is shown in Figure 5.8 for the $98 \mathrm{~nm}$ film annealed at $115{ }^{\circ} \mathrm{C}$ for 5 min. Assuming a Gaussian distribution of $h$, the standard deviation $\sigma_{\mathrm{AFM} \text {,surf }}$ was evaluated by fitting eq. (2) to the observed distribution.

$$
f(h)=\frac{1}{\sqrt{2 \pi} \sigma_{\mathrm{AFM}, \text { surf }}} \exp \left(-\frac{(h-\bar{h})^{2}}{2 \sigma_{\mathrm{AFM}, \text { surf }}^{2}}\right)
$$

where $\bar{h}$ is the average value of the height. The result of fit is shown by a solid curve in the figure. As seen in Figure 5.8 the distribution is well described by a Gaussian function in the incubation period. The evaluated surface roughness $\sigma_{\mathrm{AFM} \text {,surf }}$ is plotted in Figure 5.6 as a function of the annealing time in the incubation period to compare with the interface roughness $\sigma_{\mathrm{NR}, \text { iner }}$ evaluated by the NR measurements.

The AFM surface roughness $\sigma_{\mathrm{AFM} \text {,surf }}$ is almost independent of the annealing time. On the other hand, the interface roughness $\sigma_{\mathrm{NR} \text {,inter }}$ increases with the annealing time from the beginning of the incubation time. In the two phase region it is expected that the phase separation would occur in the middle blend layer into PVME-rich and PS-rich phases. Nevertheless, no changes were observed during incubation period in the CLSM images, suggesting that the phase separation dose not proceed at least in the 


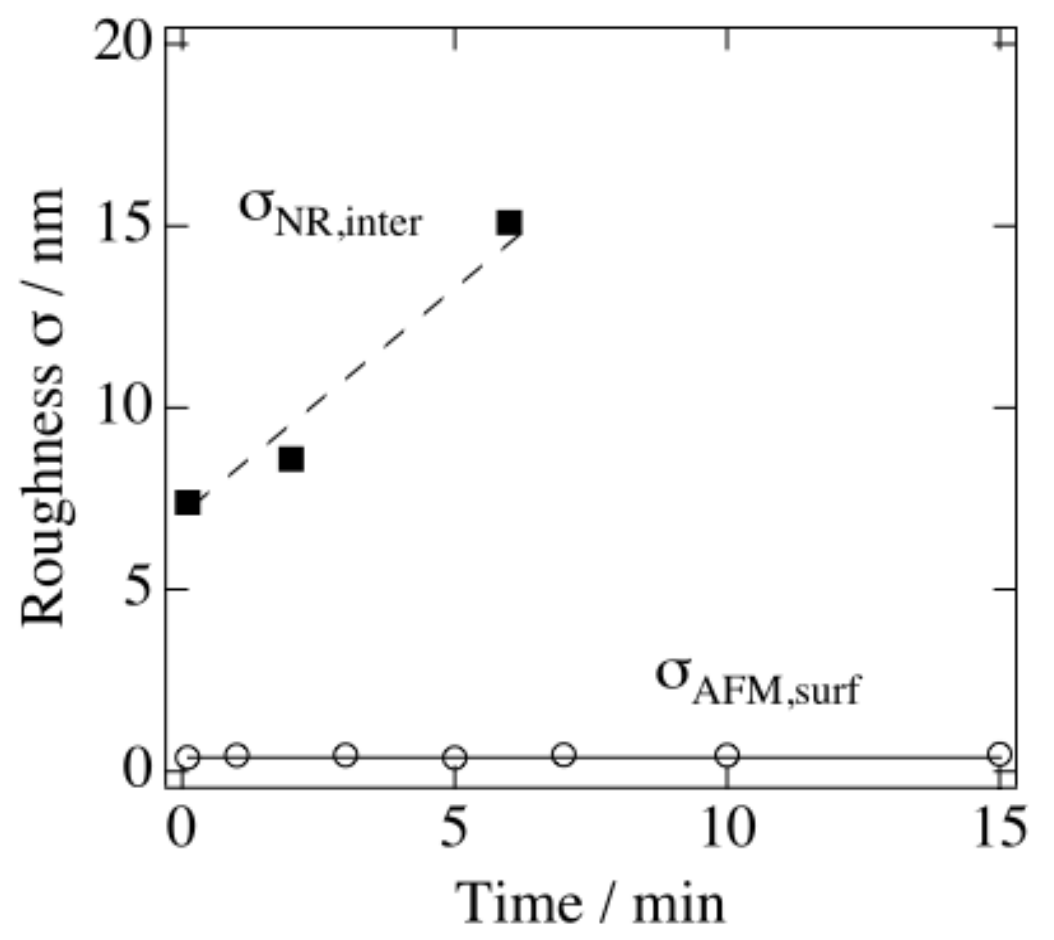

Figure 5.6: Time evolutions of interface roughness $\sigma_{\mathrm{NR} \text {,inter }}$ evaluated from the NR data and the surface roughness $\sigma_{\mathrm{AFM}, \text { surf }}$ from AFM data, respectively, for dPS/PVME blend film $98 \mathrm{~nm}$ thick annealed at $115^{\circ} \mathrm{C}$ in the two phase region. 


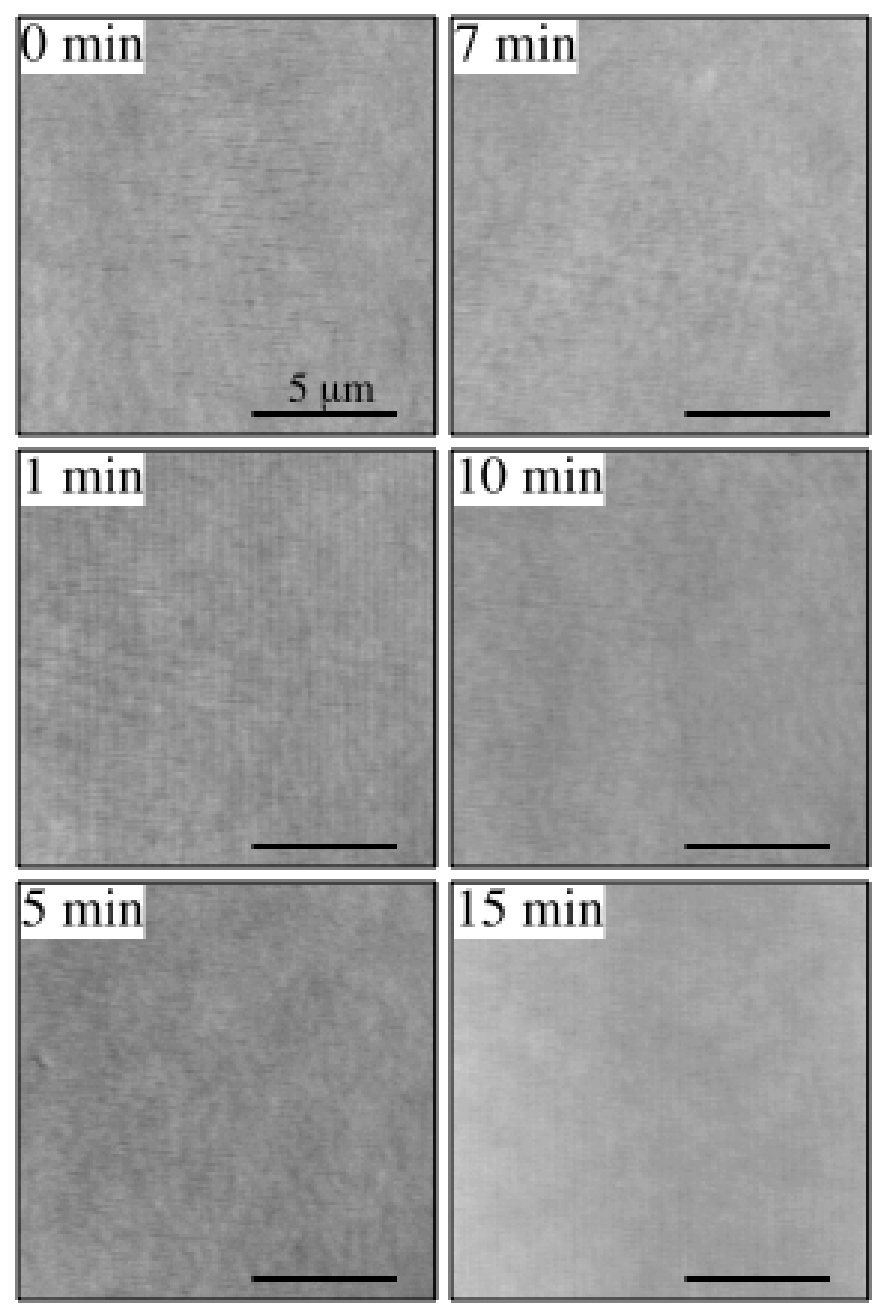

Figure 5.7: Time evolution of AFM images of $\mathrm{dPS} / \mathrm{PVME}$ blend film $98 \mathrm{~nm}$ thick after temperature jump to $115{ }^{\circ} \mathrm{C}$ in the two phase region. Scale bars in AFM images are $5 \mu \mathrm{m}$. 


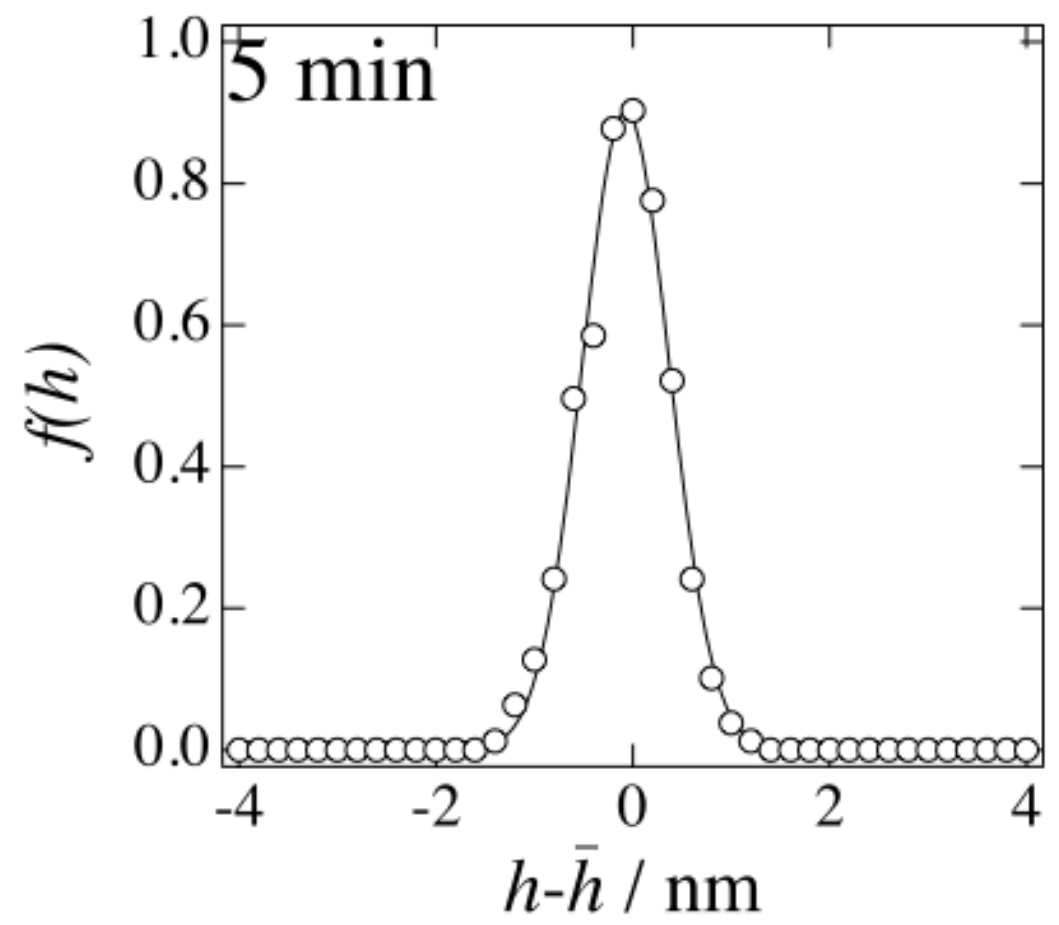

Figure 5.8: Distribution $f(h)$ of surface heights in the surface of dPS/PVME film $98 \mathrm{~nm}$ thick annealed at $115{ }^{\circ} \mathrm{C}$ for $5 \mathrm{~min}$. The solid curve is result of fit to a Gaussian function. 
in-plane direction. The increase of the NR interface roughness $\sigma_{\mathrm{NR} \text {,inter }}$ in the incubation period indicates that the phase separation proceeds in the depth direction, which must be induced and accelerated by the preferential interactions between PVME and air [16]. This phase separation in the depth direction does not occur coherently in the depth direction like the surface directed spinodal decomposition (SDSD) [20], but inhomogeneously in the in-plane direction [21].

In the following we will discuss the dewetting mechanism on the basis of the observations. In Chapter 3 we considered two possibilities for the dewetting mechanism for the PS/PVME thin films in Region IV below 200 nm: the capillary fluctuation mechanism [6, 7] and the composition fluctuation mechanism [8, 9-12]. The former mechanism was proposed by Composto et al. [6, 7] to explain the dewetting of deuterated poly(methyl methacrylate) (dPMMA) and poly(styrene-ran-crylonitrile) (SAN) in a thickness $d$ range of $R_{\mathrm{g}}<d<150 R_{\mathrm{g}}$. In order to check the mechanism we evaluated the capillary fluctuation for the interface between the surface PVME and the middle blend layer based on the theory by Shull et al. [22]. It was found that the fluctuation length was less than $3.3 \mathrm{~nm}$, which is much smaller than the interface roughness derived from the NR result in the incubation period, implying a less possibility of the capillary fluctuation mechanism.

The composition fluctuation mechanism was theoretically introduced by some researchers [9-12] and An and coworkers [8] applied this idea to explain the dewetting of thermodynamically stable blend ultrathin film of dPMMA and SAN with $d \sim R_{\mathrm{g}}$. In this mechanism polymers which have preferable interactions with the surface (or the substrate) diffuse to the surface (or the substrate) so as to create the composition gradient across the film. The diffusion could not occur homogeneously over the film surface to create the composition fluctuations in the mixture along the surface. When the amplitude of the fluctuations is large enough, the free surface is eventually destabilized, leading to the dewetting [9-12]. This mechanism is also possible in the two phase region. In the present experiment we observed tri-layer structure in the PS/PVME blend thin film $98 \mathrm{~nm}$ thick. The film was stable in the one phase region and no dewetting occurs. After the temperature jump into the two phase region, however, we found that the interface roughness $\sigma_{\mathrm{NR} \text {,inter }}$ increases rapidly with the annealing time in the incubation period, showing that the composition fluctuations proceed in the depth direction with the annealing time, and the composition fluctuations 
may proceed inhomogeneously in the in-plane direction. These inhomogeneous composition fluctuations must induce the dewetting when the amplitude is enough large that the surface is destabilized. Thus, the present observations support the composition fluctuation induced dewetting mechanism for the PS/PVME blend film below 200 nm.

Finally we would like to comment on the fringed pattern observed in the NR profile at 360 min after the temperature jump in Figure 5.5(a). The fringed pattern with a long period suggests that a very thin PVME layer is formed in a very late stage after the dewetting. The thickness evaluated by fitting is $\sim 10 \mathrm{~nm}$, which is shown in the scattering length density profile in Figure 5.5(b). Why does such a thin film is formed after the dewetting? In the incubation period the phase separation occurs mainly in the depth direction, but when the dewetting occurs at around 60 min after the temperature jump the phase separation is not completed. After the dewetting into the droplets the phase separation may proceed within the droplets. Outer surface of the droplets must be PVME because the preferential interactions between PVME and air [13-17]. The phase separation further proceeds and the surface PVME increases in thickness. Finally PVME comes out from the surface of the droplets onto the substrate to form a very thin layer $10 \mathrm{~nm}$ thick. Similar thin film formation was reported by Stamm et al [23] after a very long annealing of dPS and PpMS blend thin film.

\subsection{Conclusion}

In this study we investigated structure development of blend thin films of dPS (PS) and PVME below $200 \mathrm{~nm}$ at $115{ }^{\circ} \mathrm{C}$ in the two phase region during the incubation period before the dewetting. It was found in the NR measurements that the thin film had a tri-layer structure consisting of the surface PVME layer, the middle blend layer and the bottom PVME layer even in the one phase region. This must be due to preferable interactions of PVME and air and PVME and Si substrate. When the thin film was annealed at $115{ }^{\circ} \mathrm{C}$ the interfacial roughness $\sigma_{\mathrm{NR} \text {,inter }}$ between the top PVME and the middle blend layer rapidly increased with the annealing time. This is the phase separation of the middle layer enhanced by the preferable interaction between PVME and air. This observation supported the dewetting mechanism induced by composition fluctuations. After very long annealing of $360 \mathrm{~min}$ the NR result clearly showed that a thin PVME layer was formed on the Si substrate. It implies that the 
phase separation proceeds in the dewetted droplets, and the inner PVME comes out, and finally wets the Si surface. 


\section{References}

[1] Ebisawa T, Tasaki S, Otake Y, Funahashi H, Soyama K, Torikai N, Matsushita Y, Physica B, 213, 901 (1995).

[2] Braun C. Parratt32 program (Berlin: Berlin Neutron Scattering Center (BENSC), Hahn-Meitner Institut).

[3] Parratt L G, Phys. Rev. 95, 359 (1954).

[4] Cahn J W, Hilliard J E. J. Chem. Phys. 28, 258 (1958).

[5] Cahn J W, J. Chem. Phys. 42, 93 (1965).

[6] Wang H, Composto R J, Chem. Phys. 113, 10386 (2000).

[7] Wang H, Composto R J, Interface Science, 11, 237 (2003).

[8] Liao Y, Su Z, Sun Z, Shi T, An L, Macromol. Rapid Commun. 27, 351 (2006).

[9] Wensink K D F, Je'remoe B, Langmuir, 18, 413 (2002).

[10] Sharma A, Mittal J, Phys. Rev. Lett. 89, 1861 (2002).

[11] Sharma A, Mittal J, Verma R, Langmuir, 18, 10213 (2002).

[12] Clarke N, Macromolecules, 38, 6775 (2005).

[13] Bhatia Q, Pan D, Koberstein J, Macromolecules, 21, 2166 (1988).

[14] Cowie J, Devlin B, McEwen I, Macromolecules, 26, 5628 (1993).

[15] Lee S, Sung C, Macromolecules, 34, 599 (2001).

[16] Forrey C, Koberstein J, Pan D H, Interface Science, 11, 211 (2003).

[17] Kawaguchi D, Tanaka K, Kajiyama T, Takahara A, Tasaki S, Macromolecules, 36, 6824 (2003).

[18] Ermi B D, Karim A, Douglas J F, J. Poly. Sci., Part. B, 36, 191 (1998).

[19] Karim A, Slawecki T M, Kumar S K, Douglas J F, Satija S K, Han C C, Russell T P, Liu Y, Overnay R, Sokolov J, Rafailovich M H, Macromolecules, 31, 857 (1998).

[20] Jones R A L, Norton L J, Kramer E J, Bates F S, Wiltzius P, Phys. Rev. Lett. 66, 1326 (1991).

[21] Liao Y, Su Z, Sun Z, Shi T, An L, Macromol. Rapid Commun. 27, 351 (2006).

[22] Shull K R, Mayers A M, Russell T P, Macromolecules, 26, 3929 (1993).

[23] Mueller-Buschbaum P, O'Neil S A, Affrossman S, Stamm M, Macromolecules, 31, 5003 (1998). 


\section{Chapter 6}

\section{Time-resolved Neutron Reflectivity Measurements for Dewetting Process in Polystyrene/Poly(vinyl methyl ether) Blend Thin Films}

\subsection{Introduction}

In Chapter 5 we have studied structural development in the incubation period before the dewetting for dPS/PVME thin films below 200 nm using neutron reflectivity for the depth direction and atomic force microscopy (AFM) for the in-plane direction to elucidate the dewetting mechanism. It was found in the NR measurements that the interfacial roughness $\sigma_{\mathrm{NR} \text {,inter }}$ between the top PVME and the middle blend layer rapidly increased with the annealing time in the two phase region. This is the phase separation of the middle layer enhanced by the preferable interaction between PVME and air, which occurs in the early stage in the incubation period. It was suggested that the phase separation in the depth direction occurred inhomogeneously in the in-plane direction. In this incubation period, we concluded that the dewetting was induced by composition fluctuations. However we only measured and evaluated few depth profiles in the early stage in the incubation period in the previous NR measurements, and the measurements were conducted on quenched samples to the room temperature. For more detailed studies of the dewetting mechanism, we performed time-resolved measurements on structural development in the depth direction in the incubation period before the dewetting after temperature jump from the one phase region to the two phase region using a time-of-flight (TOF) spectrometer, and we also tried to measure the morphologies inside the dewetting droplets after the dewetting in the off-specular reflection.

\subsection{Experimental}

Deuterated polystyrene (dPS) and poly(vinyl methyl ether) (PVME) used in this study have weight-average molecular weights $M_{\mathrm{w}}=280,000$ and 90,000, and the molecular weight distributions in terms of $M_{\mathrm{w}} / M_{\mathrm{n}}=1.08$ and 1.88, respectively, where $M_{\mathrm{n}}$ is number-average molecular weight. Both of dPS and PVME were purchased 
from Scientific Polymer Products, Inc. The detailed sample preparation was written in Chapter 5. Blend films of dPS and PVME were prepared on a cleaned Si wafer with native oxide layer in the same manner as for the PS and PVME films. All the measurements were done on the blend samples with the critical concentration. Thickness of polymer film was controlled by varying the polymer concentration in solution and confirmed with ellipsometer measurements. Time-resolved neutron reflectivity measurements were performed with the Surface Profile Analysis Reflectometer (SPEAR) at the Los Alamos National Laboratory, which is a time-of-flight (TOF) reflectometer. Confocal laser scanning microscope (CLSM) measurements were done using LASER TECH. ILM15.

\subsection{Results and Discussion}

First we performed time-resolved confocal laser scanning microscope (CLSM) measurements on the dPS/PVME blend thin films after the temperature jump into the two phase region. The typical time evolution of the CLSM images are shown in Figure 6.1 for the $98 \mathrm{~nm}$ dPS/PVME blend film after the temperature jump to $125^{\circ} \mathrm{C}$. The dewetting patterns are clearly observed at around 63 min. Performing fast Fourier transform (FFT) of the images we evaluated the intensity evolution due to the dewetting to estimate the onset time of the dewetting. It was $49 \mathrm{~min}$ in the case of the $98 \mathrm{~nm}$ film at $125^{\circ} \mathrm{C}$.

Time evolution of the NR profiles for dPS/PVME 98nm blend thin film at $125^{\circ} \mathrm{C}$ in the two phase region is shown in Figure 6.2(a). The fringed pattern was clearly observed just after the temperature jump ( $t=0 \mathrm{~min}$ ), and gradually smeared with the annealing time in the early stage in the incubation period ( $\sim 50 \mathrm{~min})$. We have already shown in Chapter 5 that the interface roughness increase with the annealing time in the early stage. At $\sim 50$ min the fringed pattern is completely smeared out, showing the dewetting in the film. The NR profile calculated from the tri-layered model was fitted to the observed one to evaluate the thickness and the surface and interface roughness for each layer. It was found that the tri-layer model gave a good agreement between the observed reflectivity and the calculated one as shown by a solid curve in Figure 6.2(a). The depth profile of the evaluated scattering length density is plotted in Figure 6.2(b). In Chapter 5 the NR measurements were performed on the quenched sample. The estimated values of the interface roughness between the top PVME layer and the middle 


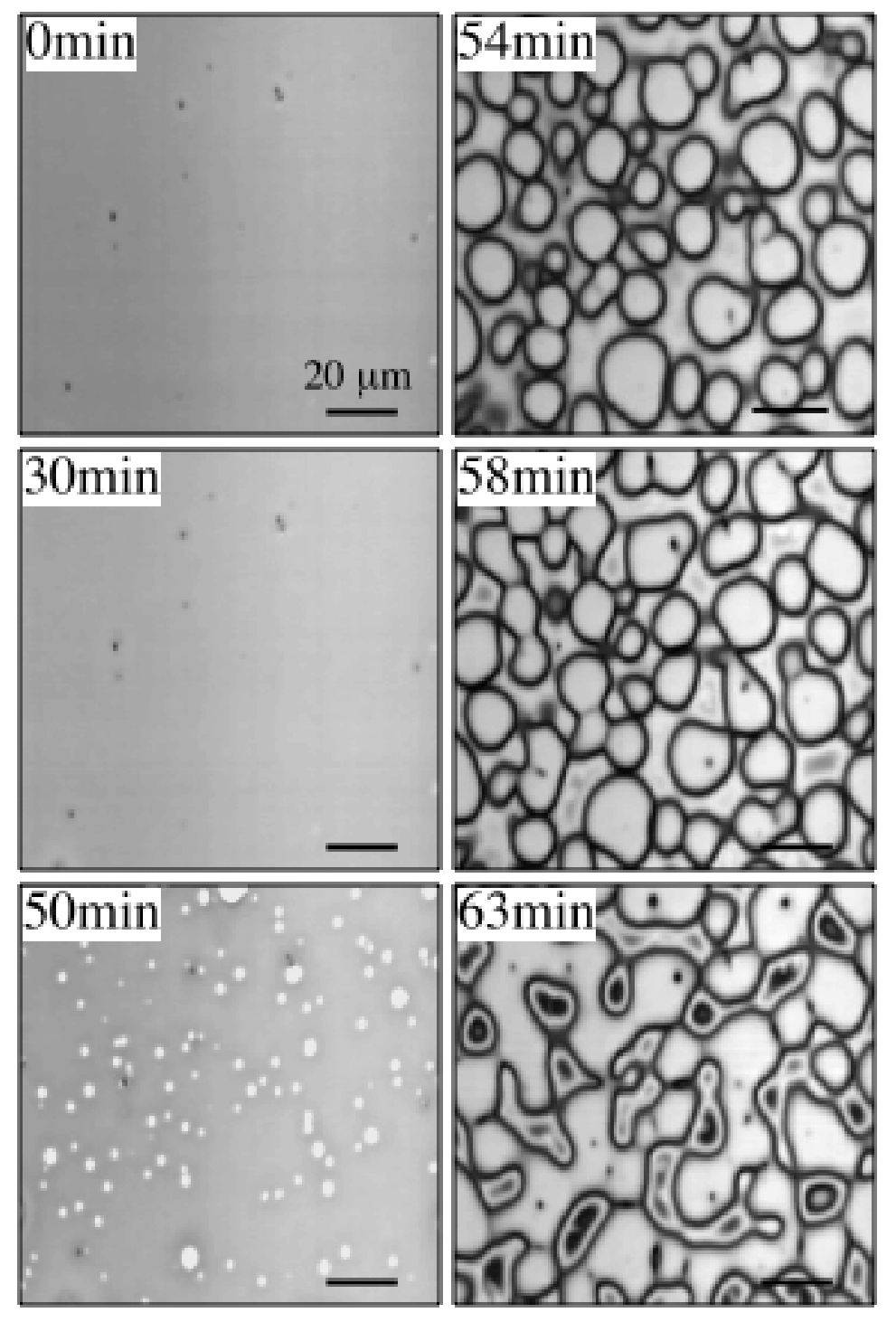

Figure 6.1: Time evolution of CLSM images of $98 \mathrm{~nm}$ $\mathrm{dPS} / \mathrm{PVME}$ blend thin film after the temperature jump to $125^{\circ} \mathrm{C}$ in the two phase region. 


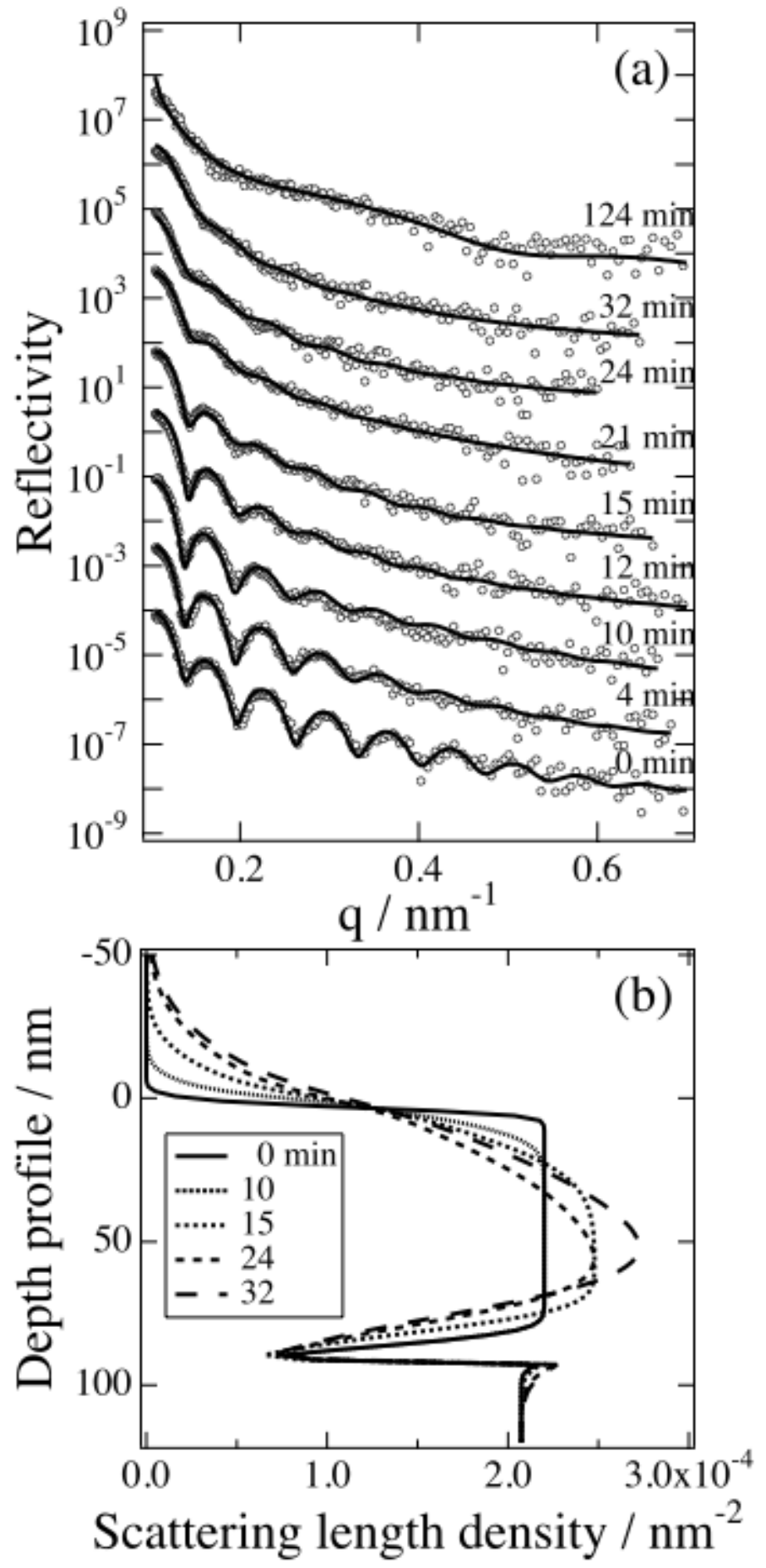

Figure 6.2: Time evolution of NR (a) and depth profile of scattering length density (b) of $98 \mathrm{~nm} \mathrm{dPS/PVME} \mathrm{blend}$ thin films after temperature jump to $125^{\circ} \mathrm{C}$. 
blend layer was $3.2 \mathrm{~nm}$ at $0 \mathrm{~min}$ in the SPEAR measurement which is close to that is 2.7 $\mathrm{nm}$ in the MINE measurement, showing the reliability of the MINE measurements on the quenched sample.

We also show the time evolution of the NR for the $42 \mathrm{~nm}$ dPS/PVME blend thin film at $125^{\circ} \mathrm{C}$ in the two phase region in Figure 6.3(a) with the results of the fits with the tri-layer model. The depth profiles of the scattering length density are shown in Figure 6.3(b) as a function of the annealing time. Qualitatively the time evolutions of the NR profiles are almost identical for the two thicknesses. However, we can see some characteristic features and/or differences in the time evolution of the depth profile. (i) Both of the interface roughness between the top PVME layer and the middle blend layer (hereafter, it terms the top interface) and between the bottom PVME layer and the middle blend layer (hereafter it terms the bottom interface) increase with the annealing time even in the incubation period before the dewetting. (ii) the two roughnesses evolve with the annealing time very asymmetrically in the depth direction for the 98 and $42 \mathrm{~nm}$ films, (iii) the roughness of the $98 \mathrm{~nm}$ film is more rapidly increases than that of the $42 \mathrm{~nm}$ film. (iv) at the late stage after dewetting, a fringed pattern with a very long period is observed for both film thicknesses (see 124 min data in Figure 6.2(a) and 159 min data in Figure 6.3(a)). In the following we will discuss these features to elucidate the mechanism of the dewetting in a thickness region of $2 R_{\mathrm{g}}$ to $\sim 200 \mathrm{~nm}$.

The time evolution of the top interface roughness is shown in Figures 6.4(a) and (b) for the $98 \mathrm{~nm}$ film and the $42 \mathrm{~nm}$ film, respectively, at three temperatures of 115, 125 and $135{ }^{\circ} \mathrm{C}$. The increase of the interface roughness is clearly observed even in the early stage in the incubation period. The increase of the roughness shows that the phase separation proceeds even in the incubation period before dewetting, which must be accelerated by the preferential interactions between PVME and air as discussed in Chapter 5. It is noted that the time evolution of the bottom interface roughness is very different from that of the top interface roughness. In order to show the difference, time evolutions of the interface roughness at $125^{\circ} \mathrm{C}$ are shown in Figure 6.5 (a) and (b) for the 98 and $42 \mathrm{~nm}$ films, respectively. The top interface roughness begins to increase with the annealing time just after the temperature jump while the bottom interface roughness stays almost constant in the beginning of the annealing and slowly increases. This result shows that the phase separation near the substrate is hindered compared with that near the surface, resulting in an asymmetric depth profiles of the 

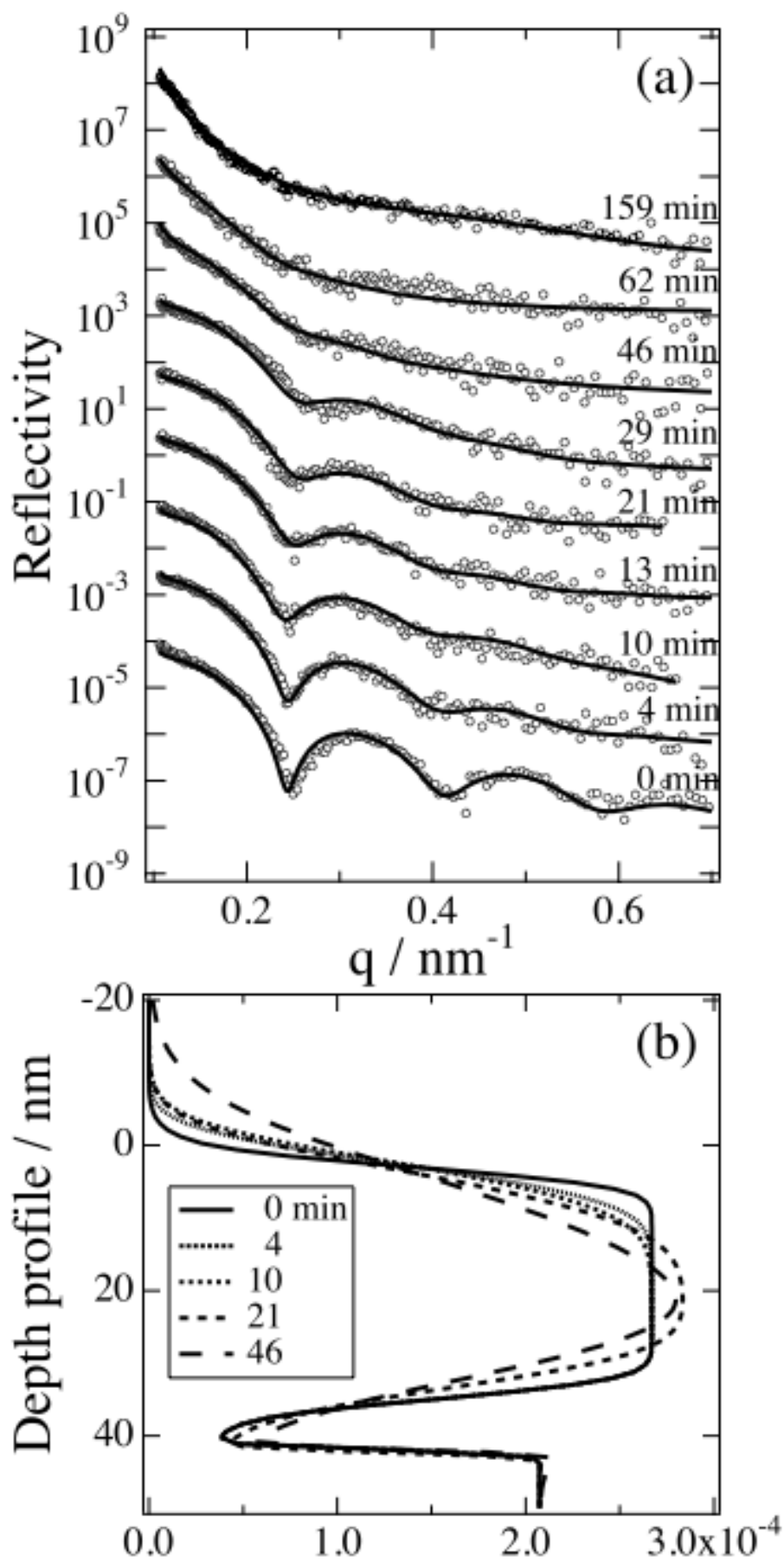

Scattering length density $/ \mathrm{nm}^{-2}$

Figure 6.3: Time evolution of NR (a) and depth profile of scattering length density (b) of $42 \mathrm{~nm}$ dPS/PVME blend thin films after temperature jump to $125^{\circ} \mathrm{C}$. 

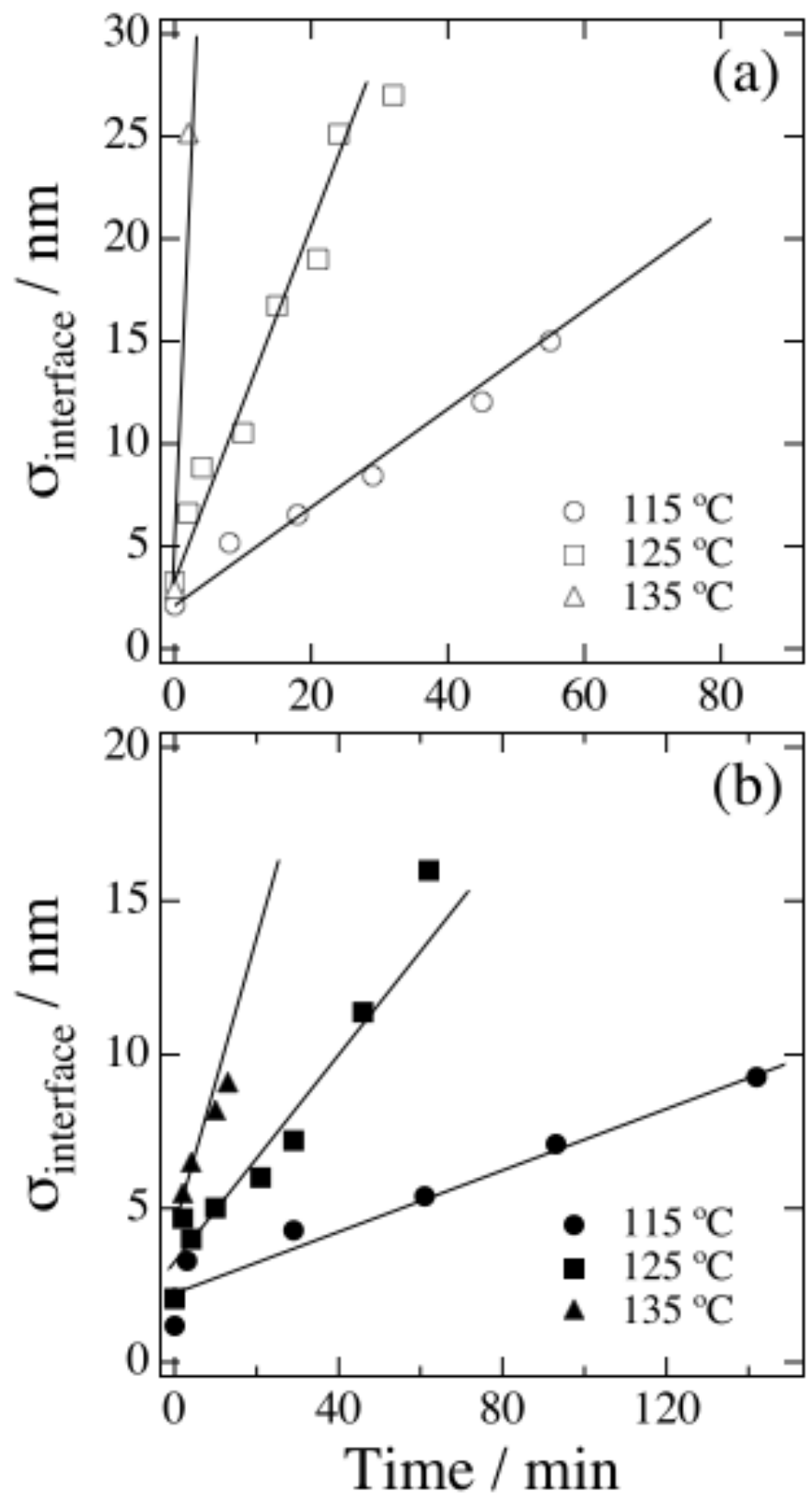

Figure 6.4: Time evolution of top interface roughness evaluated NR results for 98nm (a) and $42 \mathrm{~nm}$ (b) dPS/PVME blend thin films after temperature jump to 115. $125.135^{\circ} \mathrm{C}$. 

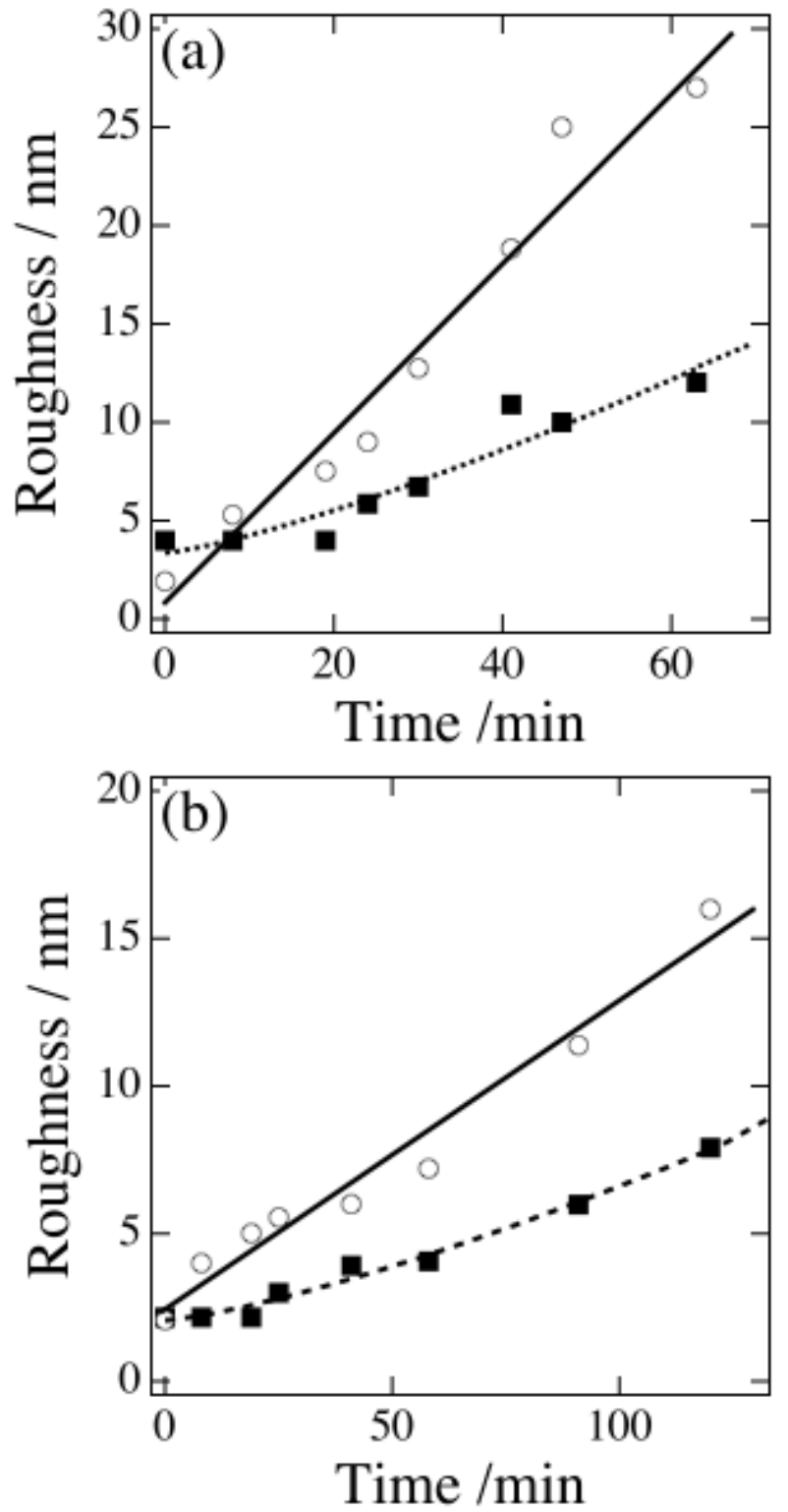

Figure 6.5: Comparison of the time evolution of interface roughness between the top interface layer and the bottom interface layer for $98 \mathrm{~nm}$ film (a) and $42 \mathrm{~nm}$ film (b) after temperature jump to $125^{\circ} \mathrm{C}$. 
scattering length. According to the phase separation near the substrate, the bottom PVME layer thickness increases with the annealing time before the dewetting. For example, the thickness of the bottom PVME layer is $\sim 5 \mathrm{~nm}$ before $10 \mathrm{~min}$, and increases to $15 \mathrm{~nm}$ at $32 \mathrm{~min}$. With increasing bottom PVME layer thickness, the scattering length density in the middle layer also increases. In the case of the $98 \mathrm{~nm}$ film the estimated PS concentration is 0.42 .

As shown above, in the early stage in the incubation period the composition fluctuation occurs only from the surface region, and in the late stage in the incubation period PVME in the middle layer separates to the bottom layer although that of the surface region still increases. This asymmetry of composition fluctuations must be caused by the difference between molecular mobility near the surface and that near the substrate. We consider that the molecular mobility near the substrate is lower than that near the surface. In other words, it is a hard wall effect on molecular mobility. Hence, the composition fluctuations near the substrate are delayed due to the lower mobility. On the other hand, the composition fluctuations near the surface region can occur without the constraint of the hard wall, and increase rapidly compared with those near the substrate. As for the hard wall effect, Tanaka et al. reported that $T_{g}$ at the solid/polymer interface was higher than that of bulk $T_{g}$ by fluorescence lifetime measurement using evanescent wave excitation [1], suggesting the low mobility near the substrate.

It was shown in Chapter 5 the fringed pattern with a long period was observed at 360 min after dewetting, implying that a thin layer was formed on the surface of the substrate. In this measurement we also observed the fringed pattern with a long period in both the 98 and $42 \mathrm{~nm}$. It appears at 124 and 159 min for the 98 and $42 \mathrm{~nm}$ films at $125^{\circ} \mathrm{C}$, respectively. This is the first observation of the onset of the thin layer after dewetting. We evaluated the thickness of the thin PVME layers. They are $13 \mathrm{~nm}$ for both the 98 and $42 \mathrm{~nm}$ films, and very close to the value evaluated in the MINE measurement (=11 nm).

Next we examine the temperature dependence of the evolution rate or the growth rate of the interface roughness or the concentration fluctuations. In the $98 \mathrm{~nm}$ and 42 nm films (see Fig. 6.4(a) (b)) the top interfacial roughness $\sigma_{\mathrm{NR}, \text { inter }}$ increases as the annealing temperature increases. It means that the phase separation rate increases and the onset time of dewetting become earlier as the temperature increases. To evaluate 
the temperature dependence of the growth rate of the interface roughness due to the concentration fluctuations we evaluated the slope of the top interface roughness vs. time in Figure 6.4 at each temperature. The slope has a dimension of diffusion coefficient so that it terms an apparent diffusion coefficient for the concentration fluctuations in this chapter. The temperature dependence of the apparent diffusion coefficient is plotted in the Arrhenius fashion in Figure 6.6 for the 98 and $42 \mathrm{~nm}$ films. It is clear that the apparent diffusion coefficient is lower in the $42 \mathrm{~nm}$ film than the $98 \mathrm{~nm}$ film. We consider that the diffusion coefficient in the bulk system is controlled by that of PS because the glass transition temperature of PS $\left(\approx 100^{\circ} \mathrm{C}\right)$ is much higher than PVME $\left(\approx-30^{\circ} \mathrm{C}\right.$ ) in this blend. Therefore we also showed the temperature dependence of the diffusion coefficient of PS in the bulk [2] in Figure 6.6 to compare with that in the thin films. The diffusion coefficients of the 98 and $42 \mathrm{~nm}$ are larger than that of PS, suggesting that these coefficients are not governed by the relaxation of PS, but the mutual diffusion between dPS and PVME dominates the growth rate of the concentration fluctuations.

Comparing the growth rates of the interface roughness between the 98 and $42 \mathrm{~nm}$ films, the rate of the $42 \mathrm{~nm}$ is slower than the $98 \mathrm{~nm}$ (see Figures 6.3(a) and (b)). This must be the hard wall effect. In the $42 \mathrm{~nm}$ film the bottom PVME layer is $3.4 \mathrm{~nm}$ while it is $5.1 \mathrm{~nm}$ for the $98 \mathrm{~nm}$ film, suggesting that the effect of the hard substrate is stronger for the $42 \mathrm{~nm}$ film than $98 \mathrm{~nm}$. Therefore, the molecular mobility near the interface is suppressed in the $42 \mathrm{~nm}$ film more than in the $98 \mathrm{~nm}$ film.

In the next step, we will discuss the results in the off-specular reflection. Figure 6.7 shows the time evolution of $2 \mathrm{D}$ spectra of dPS/PVME $42 \mathrm{~nm}$ at $135{ }^{\circ} \mathrm{C}$. In Figure 6.7(a) we see the fringed pattern along the specular position, showing the normal thin film structure in the early stage, which is described above. As the annealing proceeds the fringed pattern are smeared due to the progress of the dewetting (Figure 6.7(b)) and the off-specular scattering increases with annealing time (Figure 6.7(c)). After the dewetting, we see an scattering peak in the off-specular reflection, which must correspond to the out-of-plane grazing incidence small-angle neutron scattering (out-of-plane GISANS) along $q_{\mathrm{x}}$ direction (see Figure 6.7(d)). From physical viewpoints the peak in the off-specular reflection (or out-of-plane GISANS) shows the structure due to phase separation inside the droplets after the dewetting. This is the first observation of phase separation in the dewetted droplet. The peak position 


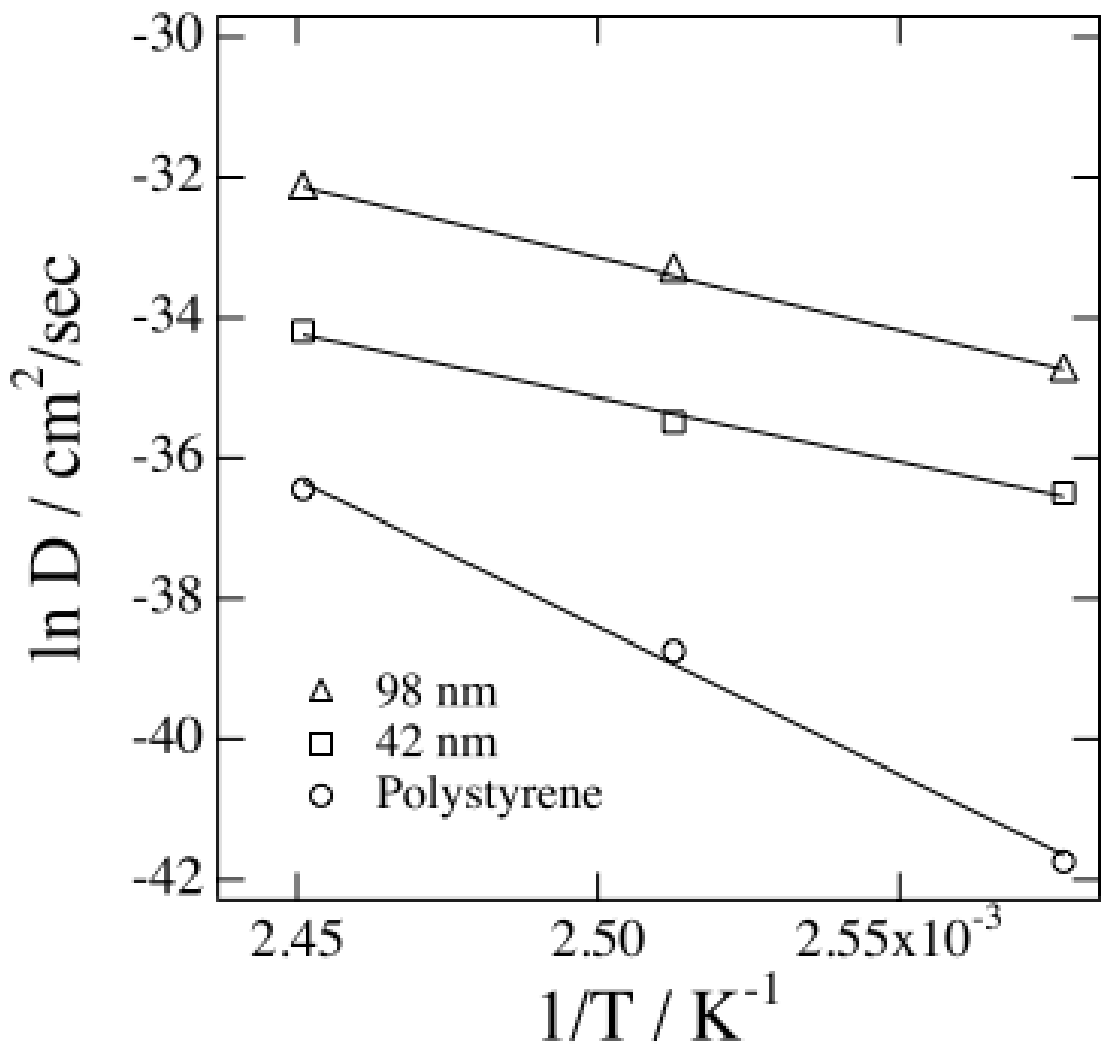

Figure 6.6: Apparent diffusion coefficients evaluated from NR results in $98 \mathrm{~nm}$ and $42 \mathrm{~nm}$ thin films and that of PS in the bulk. 

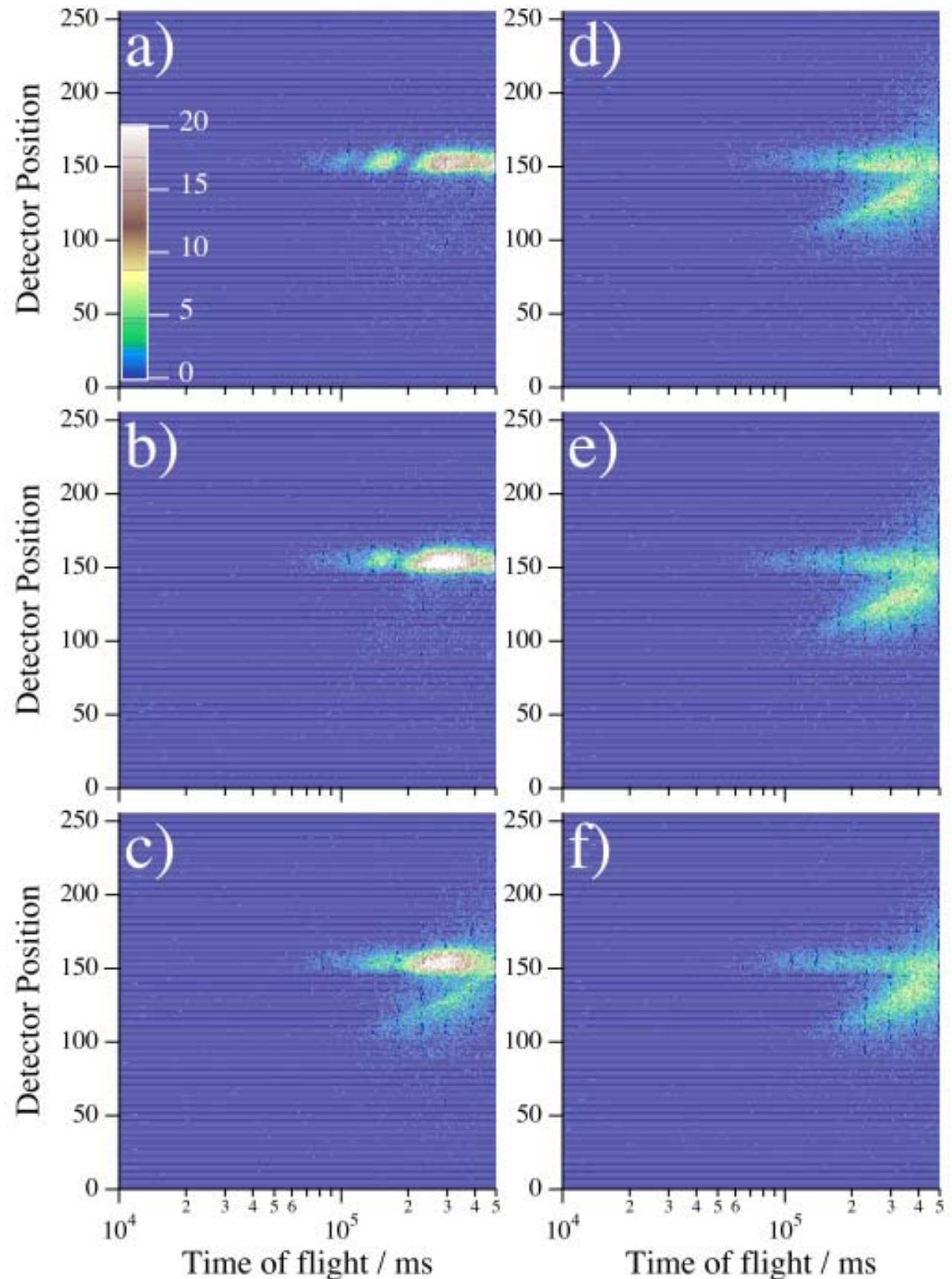

Figure 6.7: Time evolution of 2D (position and TOF) spectra of dPS/PVME $42 \mathrm{~nm}$ thin film at $135^{\circ} \mathrm{C}$ in the two phase region. 
gradually shifts to low $q_{\mathrm{x}}$ (Figures 6.7(e) and (f)), implying the growth of the domains in size within the droplets.

\subsection{Conclusion}

In this chapter we performed time-resolved NR measurements for the dewetting process in the 98 and $42 \mathrm{~nm}$ dPS/PVME blend thin films. When the thin films were annealed at $125{ }^{\circ} \mathrm{C}$ the interfacial roughness between the top PVME layer and the middle blend layer rapidly increased with the annealing time in the early stage in the incubation period. This is the phase separation of the middle layer enhanced by the preferable interaction between PVME and air. In the late stage in the incubation period, PVME in the middle blend layer also phase-separates and segregates to the bottom PVME layer with the annealing time. This asymmetric phase separation of the middle blend layer in the incubation period before dewetting could be explained in terms of a hard wall effect of the substrate, which suppresses the mobility of molecules near the substrate. As the film thickness decreases, the rate of the phase separation in the middle blend layer decreases, also showing the decrease in the mobility in the thinner films due to the hard wall effect.

In the 2D (TOF and counter position) reflectivity measurements we observed GISANS intensity in the off-specular position after the dewetting. We found that the GISANS pattern had a scattering peak after the dewetting. The peak position gradually shifted to a lower $q$, meaning the growth of the structure. This peak was assigned to a characteristic length of the phase separation in the dewetted droplets, implying that the phase separation proceeds inside the droplets after the dewetting. 


\section{References}

[1] Tanaka K, Tsuchimura Y, Akabori K, Ito F, Nagamura T, Appl. Phys. Lett. 89, 061916 (2006).

[2] Karim A, Mansour A, Felcher G P, Russell T P, Phy. Rev. B 42, 6846 (1990). 


\section{Chapter 7}

\section{Effect of Composition on Phase Separation and Dewetting in Polystyrene/Poly(vinyl methyl ether) Blend}

\subsection{Introduction}

In Chapters 3 and 4 we investigated dewetting and phase separation of polystyrene (PS) and poly(vinyl methyl ether) (PVME) blend thin films in a wide thickness range from semi-infinite thickness $(65 \mu \mathrm{m})$ to that smaller than radius of gyration $R_{\mathrm{g}}$ of the polymer chains $\left(d \sim 0.5 R_{\mathrm{g}}\right.$ ) for the critical composition of PS/PVME $=3 / 7$. We classified the phase separation and dewetting of PS/PVME blend films in the two phase region into five thickness regions. In the thickness region below about $200 \mathrm{~nm}$ and above the radius of gyration $R_{\mathrm{g}}$, it was found in Chapter 5 that the dewetting occurred in a spinodal decomposition (SD) mechanism after a long incubation time, and the dewetting was induced by composition fluctuations or the phase separation in the depth direction. This implies that the spinodal patterns in the dewetting is influenced by the phase separation. If the phase separation mechanism is not the SD type, the dewetting mechanism might be different from the SD type one. In other words, if the composition in the blend is apart from the critical one, we would expect other dewetting mechanism such as the nucleation and growth one. In fact, Karim et al. [1] studied the surface morphology of PS/PVME lend thin films with critical and off-critical compositions in the two phase region to find that the composition influences the surface morphology. In this Chapter, therefore, we studied the effects of the composition in the blend on the dewetting morphology and kinetics for PS/PVME thin films below 200 nm using optical microscope, and the structural development in the depth direction before the dewetting using neutron reflectivity.

\subsection{Experimental}

We used polystyrene (PS) and poly(vinyl methyl ether) (PVME) in this study with weight-average molecular weights $M_{\mathrm{w}}=280,000$ and 90,000, and the molecular weight distributions in terms of $M_{\mathrm{w}} / M_{\mathrm{n}}=3.01$ and 1.88, respectively, where $M_{\mathrm{n}}$ is 
number-average molecular weight. We also used deuterated polystyrene (dPS) with weight-average molecular weight $M_{\mathrm{w}}=280,000$ and the molecular weight distribution $M_{\mathrm{w}} / M_{\mathrm{n}}=1.08$ for neutron reflectivity measurements as in Chapter 5 . The detail sample preparation was written in Chapter 5 and we prepared $98 \mathrm{~nm}$ thin films with the PS compositions of 0.1, 0.3, 0.4, $0.5 \mathrm{wt} \%$.

Optical microscope (OM) measurements were done after temperature jump from one phase region to two phase region $\left(=125^{\circ} \mathrm{C}\right)$ using OLYMPUS BX50 equipped with a CCD camera. Neutron reflectivity measurements were done on MINE-II reflectometer at JRR-3 reactor in Tokai [2].

\subsection{Results and Discussion}

The OM images of PS/PVME blend thin films with the concentration of $\phi_{\mathrm{PS}}=0.1$ wt \%, $0.3 \mathrm{wt} \%, 0.4 \mathrm{wt} \%$ and $0.5 \mathrm{wt} \%$ after temperature jump to $125{ }^{\circ} \mathrm{C}$ in two phase region are shown in Figure 7.1. In the critical concentration of $\phi_{\mathrm{PS}}=0.3$ (Figure 7.1(b)), bicontinuous morphologies are observed after the incubation period in the OM images, and finally become isolated droplets at 45 min similar to the result in Chapter 3. On the other hand, in the off-critical concentration of $\phi_{\mathrm{PS}}=0.1$, some holes are formed inhomogeneously in the in-plane direction after a long incubation period (Figure 7.1(a)). These holes increase rapidly in size and number within few minutes, and finally become isolated droplets. The morphology change suggests that the dewetting proceeds in the nucleation and growth (NG) mechanism, but not the SD type one. Note that the final size of droplets is different from that for the critical composition. In the dewetting process with the off-critical composition of $\phi_{\mathrm{PS}}=0.4$ shown in Figure 7.1(c), some holes are formed inhomogeneously in the in-plane direction and grow in size with the annealing time after an incubation period of about $50 \mathrm{~min}$, which is shorter than that for $\phi_{\mathrm{PS}}=0.1$. Qualitatively, the dewetting process is similar to the case of $\phi_{\mathrm{PS}}=0.1$, suggesting that the dewetting mechanism is the NG type. However, quantitatively the time evolution of morphology is different: the growth rate of the hole size for $\phi_{\mathrm{PS}}=0.4$ is slower than that for $\phi_{\mathrm{PS}}=0.1$, but the number of the holes increases with the annealing time for $\phi_{\mathrm{PS}}=0.1$, but not for $\phi_{\mathrm{PS}}=0.4$.

As the PS concentration further increases to $\phi_{\mathrm{PS}}=0.5$, no dewetting was observed even at $180 \mathrm{~min}$ after the temperature jump into the two phase region. At last, a morphology with very weak contrast was observed at 300 min in the two phase region, 
(a)
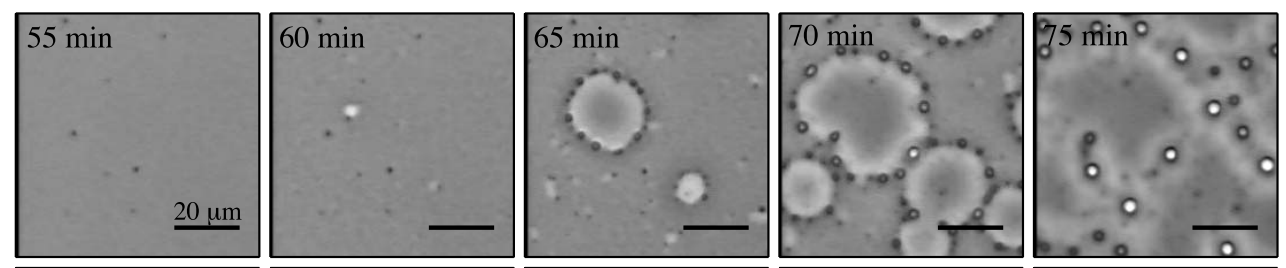

(b)
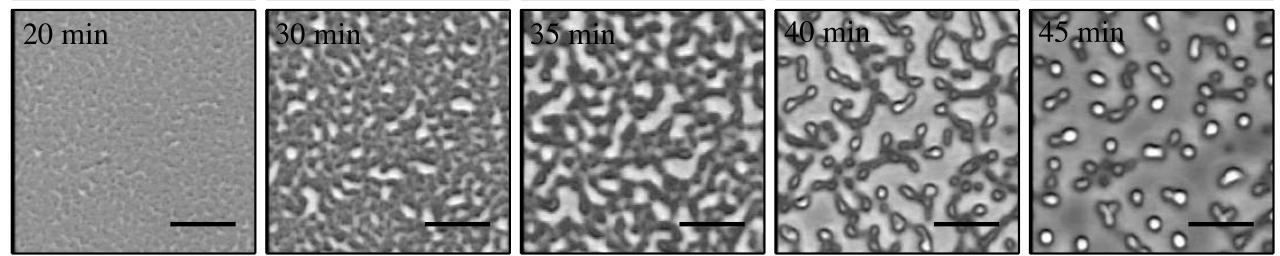

(c)
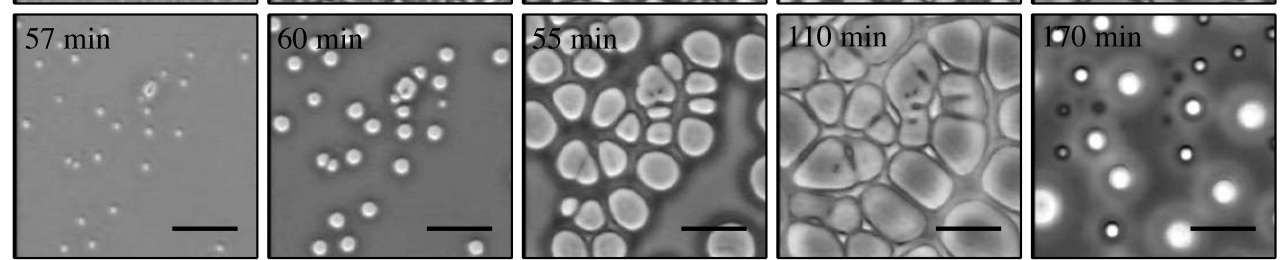

(d)
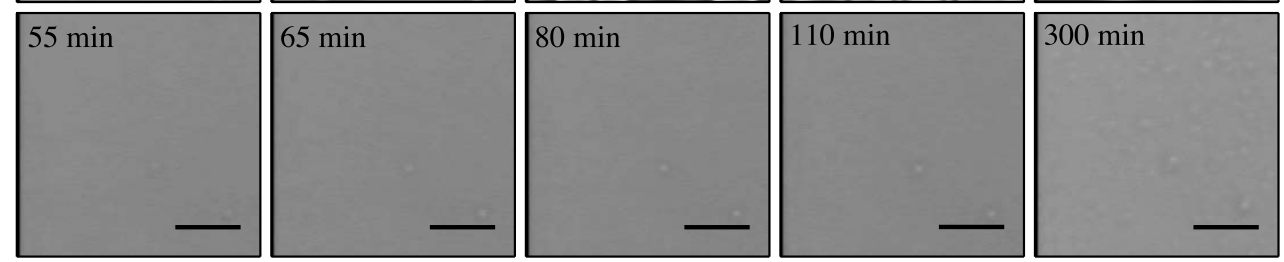

Figure 7.1: Time evolution of OM images of PS/PVME blend $98 \mathrm{~nm}$ thin films after jump to $125^{\circ} \mathrm{C}$ with (a) 10/90, (b) 30/70, (c) 40/60, (d) 50/50 composition. Scale bar is $20 \mu \mathrm{m}$. 
showing very slow kinetics for $\phi_{\mathrm{PS}}=0.5$. This may be due to the high viscosity of the blend.

These results suggest that the composition in the blend influences the morphology and kinetics of dewetting in the PS/PVME blend. For single component thin films, there are two dewetting mechanisms; one is spinodal decomposition type and another is nucleation and growth type [3]. These mechanisms are explained from the free energy of thin film as the function of thickness. In thick films, the dewetting proceeds from metastable region through the NG mechanism, on the other hand, in thin films it proceeds from unstable region through the SD mechanism. However, we found that dewetting patterns were different for the blend films with the same thickness. Hence we considered that phase separation influenced the dewetting pattern. In the critical composition, the regular bicontinuous patterns in the dewetting process suggest that the dewetting process proceeds through the SD mechanism of phase separation. On the other hand, the hole formation in the off-critical composition suggests that the dewetting process proceeds through the NG mechanism of phase separation. We have explained that the dewetting might be induced by phase separation in Chapter 3. In Chapter 5 we also showed that the dewetting in the thickness region between $\sim 20 \mathrm{~nm}<\mathrm{d}<\sim 200 \mathrm{~nm}$ is induced by the composition fluctuations in the depth direction in the incubation period. Hence we consider that the SD and ND type mechanisms in the dewetting process are dominated by the lateral phase separation process.

In order to confirm the composition fluctuations in the depth direction before dewetting for the $98 \mathrm{~nm}$ films with critical and off-critical compositions, we performed NR measurements at room temperature after annealing at $125^{\circ} \mathrm{C}$ in the two phase region. In Figure 7.2(a) the observed NR profiles of the $98 \mathrm{~nm}$ films with critical composition ( $\phi_{\mathrm{dPS}}=0.3$ ) are shown for various annealing periods. The results were fitted with the three-layer model and the results of fits are also shown by solid curves in Figure 7.2(a). Figure 7.2(b) indicates the depth profile of neutron scattering length density for various annealing periods. The fringed pattern is gradually smeared with the annealing time even in the incubation period ( $30 \mathrm{~min}$ ), suggesting that the surface roughness and/or the interface roughness increases with the annealing time before the dewetting. At 120 min the fringed pattern is completely smeared out, showing the film is dewetted as confirmed by the OM. With further annealing for 360 minutes, the fringed pattern with a long period was again observed. 

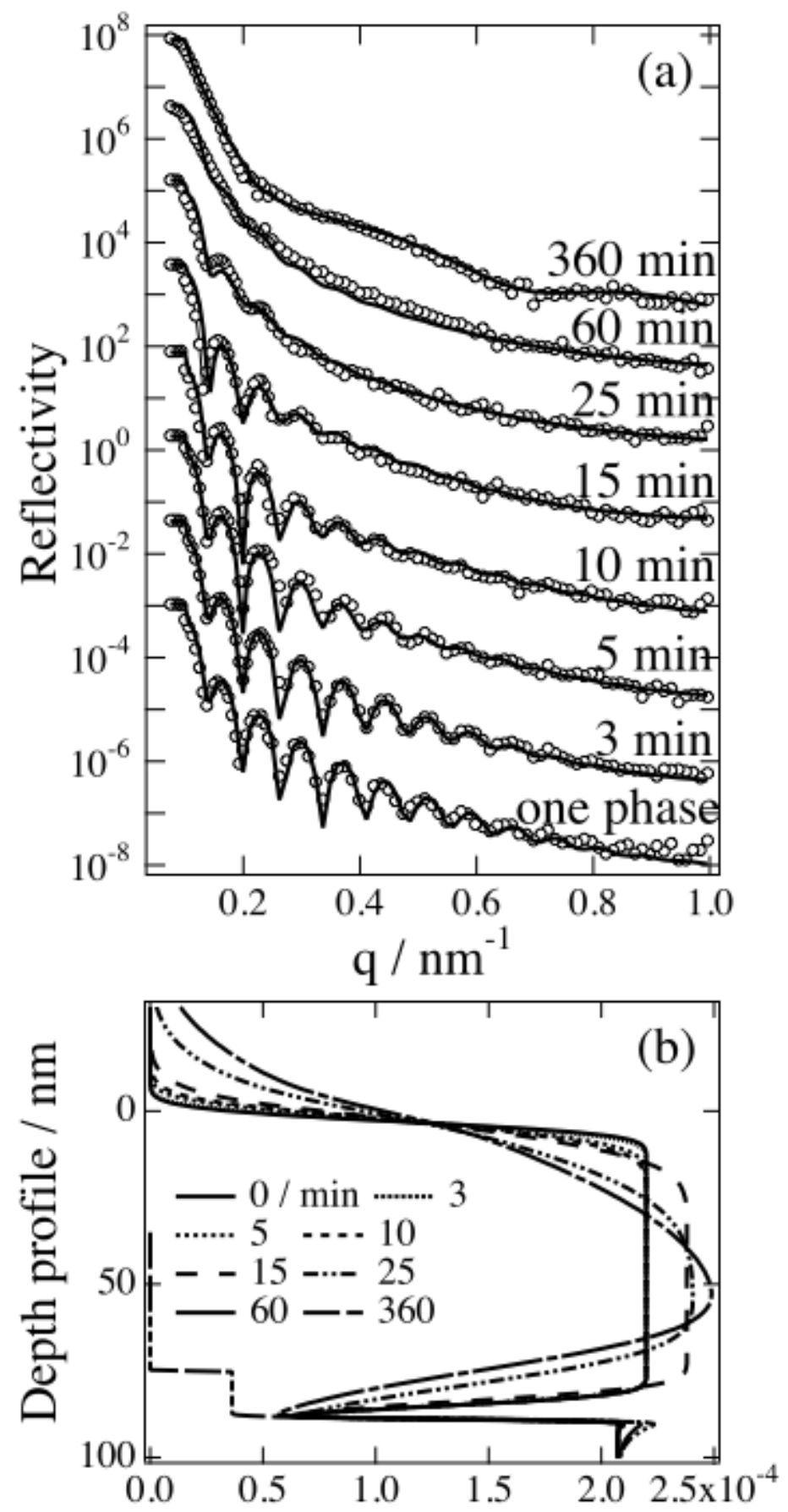

Scattering length density $/ \mathrm{nm}^{-2}$

Figure 7.2: Time evolution of NR and depth profile of PS/PVME blend $98 \mathrm{~nm}$ thin films with off-critical composition (40/60) after jump to $125^{\circ} \mathrm{C}$. 
In Figure 7.3 (a), we also showed the NR profiles for the $98 \mathrm{~nm}$ films with off-critical composition $\left(\phi_{\mathrm{dPS}}=0.4\right)$ for various annealing periods. The NR profiles were also fitted with the tri-layer model and the results are shown in Figure 7.3(a) by solid curves. Figure 7.3(b) shows the depth profiles of the scattering density evaluated from the fits. The fringed pattern is not significantly smeared with the annealing time for $30 \mathrm{~min}$, and after the incubation period it is gradually smeared with the annealing time. With further annealing for 360 minutes, the fringed pattern was again observed. The thickness of PVME layer evaluated by fitting is $\sim 10 \mathrm{~nm}$, which is the same thickness as that in the critical composition and the scattering length density profiles evaluated here are shown in Figure 7.3 (b).

In the fits we estimated the interface roughness $\sigma_{\mathrm{NR} \text {,inter }}$ and the surface roughness $\sigma_{\mathrm{NR} \text {,surf }}$ in the dewetting processes for the critical and off-critical compositions. The interface roughness $\sigma_{\mathrm{NR} \text {,inter }}$ is plotted as a function of the annealing time in Figure 7.4. We also showed the onset times of dewetting in the in-plane direction determined from OM results for the critical and off critical compositions in Figure 7.4, which are 17 and 55 minutes, respectively. The NR interface roughness $\sigma_{\mathrm{NR} \text {,inter }}$ increases for both the critical and off-critical compositions in the incubation period of the dewetting, implies that the phase separation proceeds in the depth direction. However, the roughness $\sigma_{\mathrm{NR} \text {,inter }}$ increases in very different manners between the critical and the off-critical compositions. In the critical composition, $\sigma_{\mathrm{NR} \text {,inter }}$ begins to increase just after the temperature jump, but in the off-critical composition $\sigma_{\mathrm{NR} \text {,inter }}$ increases very slowly with annealing time before $30 \mathrm{~min}$ and begins to increase rapidly after $30 \mathrm{~min}$, showing that the mechanisms of the compositional fluctuations are different between critical and off-critical compositions in the incubation period. In the following we will discuss the dewetting mechanism on the basis of the observations.

In the critical composition, the increase of NR interface roughness $\sigma_{\mathrm{NR} \text {,inter }}$ in the incubation period suggests that the phase separation proceeds in the depth direction, though we do not observe the phase separation in the OM and LS profiles in the early stage in the incubation period. On the other hand, we see very weak SD-like pattern in the OM image and slight increase in the LS intensity in the late stage in the incubation period, which was discussed in Chapter 5. We suggested that the composition fluctuations in the depth direction occur heterogeneously in the in-plane direction. In 

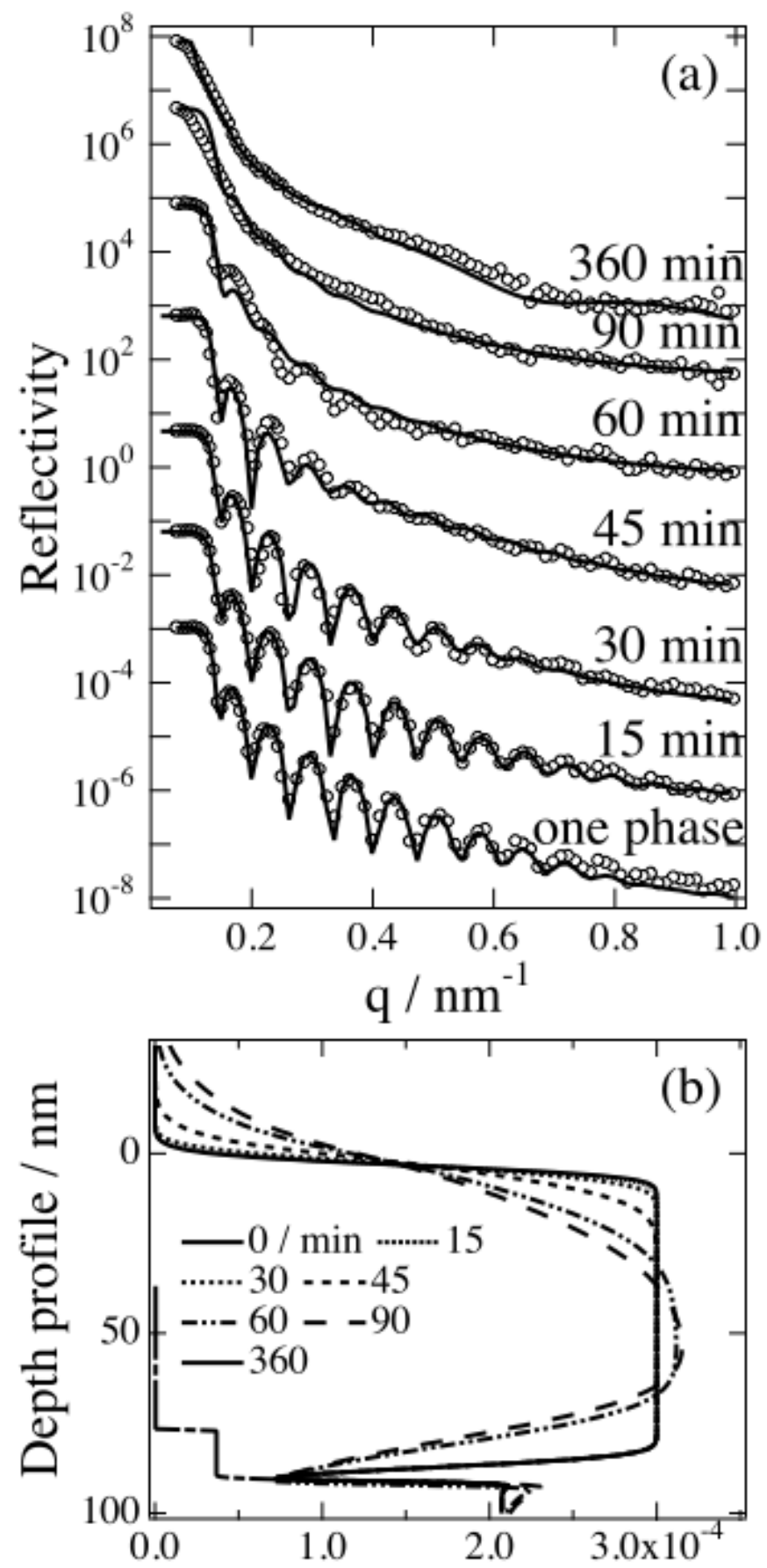

Scattering length density $/ \mathrm{nm}^{-2}$

Figure 7.3: Time evolution of NR and depth profile of PS/PVME blend $98 \mathrm{~nm}$ thin films with off-critical composition (40/60) after jump to $125^{\circ} \mathrm{C}$. 


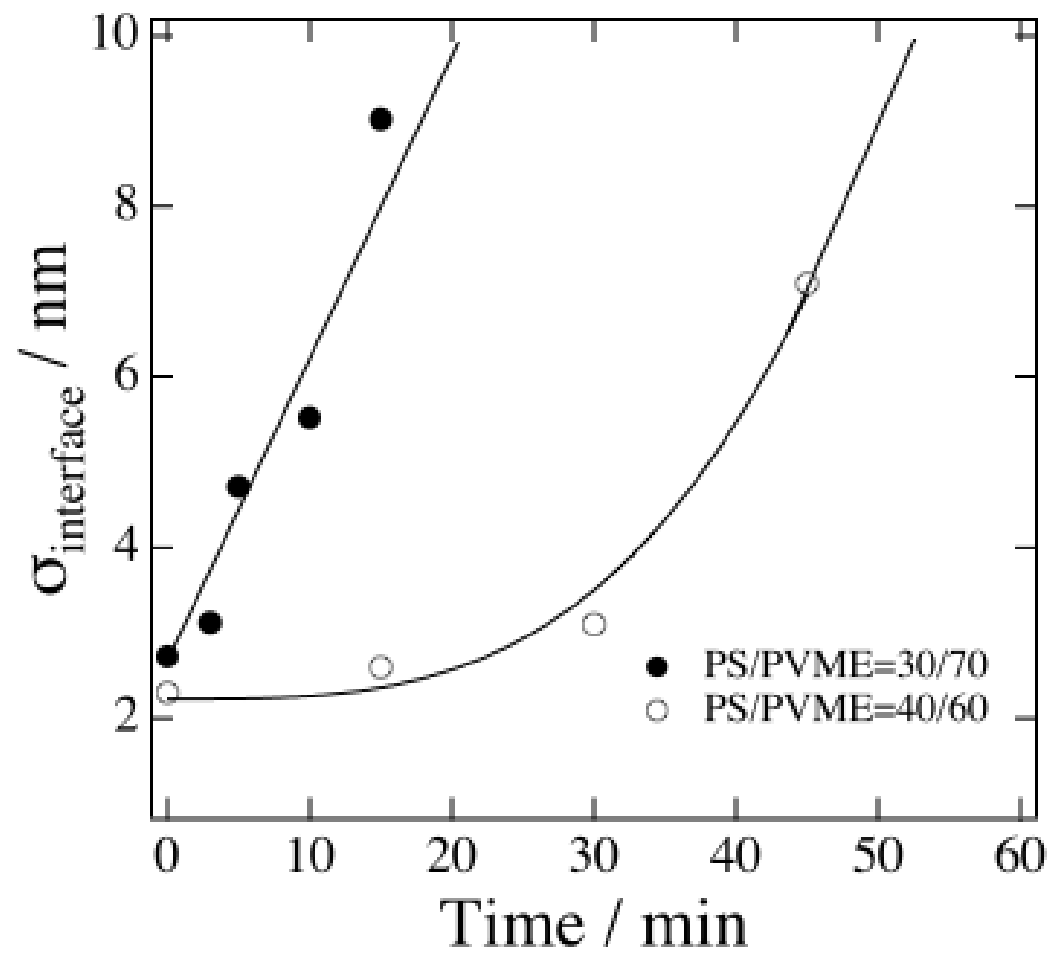

Figure 7.4: Time evolution of interface roughness evaluated from NR data of PS/PVME blend $98 \mathrm{~nm}$ thin films after jump to $125^{\circ} \mathrm{C}$ with critical and off-critical composition. 
the off-critical composition, as mentioned above, the NR interface roughness $\sigma_{\mathrm{NR} \text {,inter }}$ hardly increases in the early stage before $\sim 30 \mathrm{~min}$ and rapidly increases after $\sim 30 \mathrm{~min}$. Here it should be noted that these increases are in the incubation period of the dewetting. These observations suggests that the phase separation in the depth direction has an incubation period, suggesting that the phase separation proceeds in the NG mechanism. The NG type composition fluctuations may induce the NG type dewetting when the amplitude is enough large to destabilize the film. On the other hand, in the critical composition the composition fluctuations in the depth direction occur without the incubation time, suggesting that they proceed from the unstable region or in the SD mechanism. Therefore the interface roughness $\sigma_{\mathrm{NR} \text {,inter }}$ increases even from the early stage in the incubation period of the dewetting. The dewetting may be influenced by the composition fluctuations in the depth direction, and the SD type and NG type dewetting are observed respectively for the critical and off-critical compositions.

\subsection{Conclusion}

In this Chapter, we have studied structure development of 98nm blend thin films of PS and PVME at $125{ }^{\circ} \mathrm{C}$ in the two phase region with the critical and off-critical compositions. It was found in the $\mathrm{OM}$ observations that the dewetting process proceeds through the SD mechanism for the critical composition. On the other hand, the dewetting process proceeds through the NG mechanism for the off-critical composition. These observations suggest that the phase separation before dewetting affects the dewetting mechanism. In order to confirm the idea the NR measurements have been performed to investigate the structure development in the depth direction before dewetting for the critical and off-critical compositions. It was found that the interface roughness $\sigma_{\mathrm{NR} \text {,inter }}$ between the top PVME and the middle layer rapidly increased with annealing time just after the temperature jump for the critical composition. On the other hand, the interface roughness $\sigma_{\mathrm{NR} \text {,inter }}$ for the off-critical composition hardly increased in the early stage and rapidly increased in the late stage in the incubation period of the dewetting. These results may suggest that phase separation in the depth direction occurs in the SD and NG type mechanisms for the critical and off-critical compositions, respectively. It is considered that the dewetting mechanisms were affected by the phase separation or the compositional fluctuations before dewetting in the depth direction, resulting in the SD ad NG type for the critical 
and off-critical compositions, respectively. 


\section{References}

[1] Ermi B D, Karim A, Douglas J F, J. Poly. Sci., Part. B, 36, 191 (1998).

[2] Ebisawa T, Tasaki S, Otake Y, Funahashi H, Soyama K, Torikai N, Matushita Y, Physica B, 213\&214, 901 (1995).

[3] De Gennes P, Quere D, Brochard-Wyart F, Capillarity and Wetting Phenomena: Drops, Bubbles, Pearls, Waves: Springer (2003). 


\section{References}

[1] Ermi B D, Karim A, Douglas J F, J. Poly. Sci., Part. B, 36, 191 (1998).

[2] Ebisawa T, Tasaki S, Otake Y, Funahashi H, Soyama K, Torikai N, Matushita Y, Physica B, 213\&214, 901 (1995).

[3] De Gennes P, Quere D, Brochard-Wyart F, Capillarity and Wetting Phenomena: Drops, Bubbles, Pearls, Waves: Springer (2003). 


\section{Summary}

This thesis includes studies on phase separation and dewetting of polymer blend thin films. The contents of the respective chapters are summarized below.

In Chapter 1, the author briefly reviewed the mechanisms of nucleation and growth type phase separation and spinodal decomposition type one and also dewetting, and described the current situation of studies on polymer blend thin films.

In Chapter 2, the author described the fundamental theories of scattering and reflectivity. In addition various apparatus used in this thesis were briefly explained mainly focusing on the small-angle light scattering.

In Chapter 3, we investigated the morphology and the kinetics of phase separation and dewetting in blend thin films of PS/PVME as a function of film thickness from 40 $\mathrm{nm}$ to $65 \mu \mathrm{m}$ using $\mathrm{OM}$ and AFM as well as LS. It could be classified into four thickness regions (Regions I, II, III and IV). In Region I above $\sim 15 \mu \mathrm{m}$, the phase separation kinetics can be explained as normal SD phase separation in bulk. As the film thickness decreases, the characteristic wavelength to the SD phase separation becomes smaller with the film thickness in Region II between $\sim 15$ and $\sim 1 \mu$ m because the composition fluctuations are suppressed by the film thickness. In Region IV below $\sim 200 \mathrm{~nm}$ the films dewet after a long incubation time. In Region III (1 $\mu \mathrm{m} \sim 200 \mathrm{~nm})$ between Region II and Region IV the phase separation and dewetting occur simultaneously and their characteristic wavelengths are mixed up.

In Chapter 4, the morphology and the kinetics of the PS/PVME blend thin films below about twice radius of gyration were studied. It was found that the dewetting occurred to form droplet structure in the thickness region below $13 \mathrm{~nm}$ when the film was prepared by spin-coating the solution even in the one phase region. The SNOM measurements were also done on the dewetted structure to see if the phase separation occurred inside the droplets or not using perylene labeled PS. It was found that PS was inside the dewetted droplet. Comparing the SNOM and the AFM data it was found that the outer PVME layer was in the order of $\sim 50 \mathrm{~nm}$.

In Chapter 5, we studied structure development of blend thin films of dPS (PS) and PVME below $\sim 200 \mathrm{~nm}$ at $115{ }^{\circ} \mathrm{C}$ in the two phase region during the incubation period before the dewetting. It was found in the NR measurements that the thin film had a 
tri-layer structure consisting of the surface PVME layer, the middle blend layer and the bottom PVME layer even in the one phase region. This must be due to preferable interactions of PVME and air and PVME and Si substrate. When the thin film was annealed at $115{ }^{\circ} \mathrm{C}$ the interfacial roughness $\sigma_{\mathrm{NR} \text {,iner }}$ between the top PVME and the middle blend layer rapidly increased with the annealing time. This is the phase separation of the middle layer enhanced by the preferable interaction between PVME and air. This observation supported the dewetting mechanism induced by composition fluctuations.

In Chapter 6, time-resolved NR measurements for the dewetting process in the 98 and $42 \mathrm{~nm} \mathrm{dPS} / \mathrm{PVME}$ blend thin films have been investigated. When the thin films were annealed in the two phase region the interfacial roughness between the top PVME layer and the middle blend layer rapidly increased with the annealing time in the early stage in the incubation period. This is the phase separation of the middle layer enhanced by the preferable interaction between PVME and air. In the late stage in the incubation period, PVME in the middle blend layer also phase-separates and segregates to the bottom PVME layer with the annealing time. This asymmetric phase separation of the middle blend layer in the incubation period before dewetting could be explained in terms of a hard wall effect of the substrate, which suppresses the mobility of molecules near the substrate. As the film thickness decreases, the rate of the phase separation in the middle blend layer decreases, also showing the decrease in the mobility in the thinner films due to the hard wall effect.

In the $2 \mathrm{D}$ (TOF and counter position) reflectivity measurements we found that the GISANS pattern had a scattering peak after the dewetting. The peak position gradually shifted to a lower $q$ with annealing time, meaning the growth of the structure. This peak was assigned to a characteristic length of the phase separation in the dewetted droplets, implying that the phase separation proceeds inside the droplets after the dewetting.

In Chapter 7, we studied structure development of 98nm blend thin films of PS and PVME with the critical and off-critical compositions in the two phase region. It was found that the dewetting process proceeded through the SD mechanism for the critical composition. On the other hand, the dewetting process proceeded through the NG mechanism for the off-critical composition. In order to confirm the idea the NR measurements have been performed to investigate the structure development in the 
depth direction before dewetting for the critical and off-critical compositions. It was found that the interface roughness $\sigma_{\mathrm{NR} \text {,iner }}$ between the top PVME and the middle layer rapidly increased with annealing time just after the temperature jump for the critical composition. On the other hand, the interface roughness $\sigma_{\mathrm{NR} \text {,iner }}$ for the off-critical composition hardly increased in the early stage and rapidly increased in the late stage in the incubation period of the dewetting. These results may suggest that phase separation in the depth direction occurs in the SD and NG type mechanisms for the critical and off-critical compositions, respectively. It is considered that the dewetting mechanisms were affected by the phase separation or the compositional fluctuations before dewetting in the depth direction, resulting in the SD ad NG type for the critical and off-critical compositions, respectively. 


\section{List of Publications}

“High Resolution Small-Angle Light Scattering Instrument for Soft Material Study”

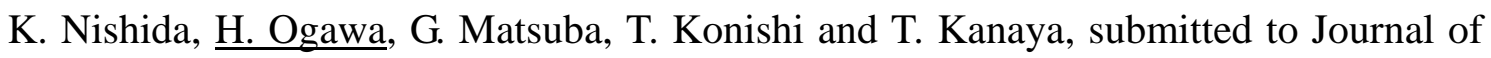
Applied Crystallography. (Chapter 2)

"Phase separation and dewetting in polymer blend thin films"

H. Ogawa, T. Kanaya, K. Nishida and G. Matsuba, Eur. Phys. J. ST, 141, 189 (2007). (Chapter 3)

"Phase separation and dewetting in polystyrene/poly(vinyl methyl ether) blend thin films in a wide thickness range"

H. Ogawa, T. Kanaya, K. Nishida and G. Matsuba, Polymer, 49, 254 (2008). (Chapter 3)

"Phase Separation and Dewetting for Ultra-thin Films in Polystyrene/Poly(vinyl methyl ether) Blend"

H. Ogawa, Y. Echizenya, T. Kanaya, R. Sekine, H. Aoki and S. Ito, to be submitted to Langmuir. (Chapter 4)

“Composition Fluctuations before Dewetting in Polystyrene/Poly(vinyl methyl ether) Blend Thin Films"

H. Ogawa, T. Kanaya, R. Inoue, K. Nishida, G. Matsuba and M. Hino, Polymer, in press. (Chapter 5)

“Time-resolved Neutron Reflectivity Measurements for Dewettnig Process in Polystyrene/Poly(vinyl methyl ether) Blend Thin Films”,

H. Ogawa, T. Kanaya, J. P. Majewski, K. Nishida, G. Matsuba, to be submitted to Journal of Chemical Physics. (Chapter 6)

\section{Patent}

K. Nishida, T. Kanaya, G. Matsuba, H. Ogawa and T. Konishi

"Instrument for Light Scattering Measurement"

Japanese Patent Pending, 2005, 2005-058211.

\section{Acknowledgement}


The present thesis is based on the studies carried out under the direction of Professor Toshiji Kanaya, Laboratory of Polymer Materials Science, Division of Fundamental Materials Properties, Institute for Chemical Research, Kyoto University, from 2004 to 2008.

The author would like to express his most sincere thanks to Professor Toshiji Kanaya for his kind guidance, encouragement, valuable comments, discussions and collaborations.

Professor Shinzaburo Ito and Professor Yoshikazu Takigawa are especially acknowledged for their critical review of this thesis.

The author wishes to express his gratitude to Associate Professor Koji Nishida for his helpful guidance, discussions and collaborations throughout this investigation.

The author wishes to express sincere thanks to Dr. Go Matsuba for his helpful guidance and advise.

The author is sincerely grateful to Associate Professor Masahiro Hino for the kind support in neutron reflectivity measurements with MINE-II at JRR-3M, Tokai. The author is grateful to Dr. Jaroslaw P. Majewski and Dr. Erik Watkins for their kind support in neutron reflectivity measurements with SPEAR at LANSCE facility, Los Alamos National Laboratory, USA. The author is sincerely grateful to Associate Professor Naoya Torikai for his kind support in the measurements at LANSCE facility and for his kind guidance and encouragement. The author is sincerely grateful to Professor Shinzaburo Ito, Associate Professor Hiroyuki Aoki and Mr. Ryojun Sekine for their kind support in scanning near-optical microscopy measurements. The author is sincerely grateful to Professor Yoshinobu Tsujii and Dr. Takashi Morinaga for their kind support in ellipsometry measurements.

The author especially wishes to express thanks to Dr. Takahiko Kawai, Dr. Nobuaki Takahashi, Dr. Takashi Konishi, Dr. Rintaro Inoue, Mr. Tsuyoshi Tsubouchi, Mr. Toru Iseki and Mr. Yuki Echizenya for their continuous support throughout this study at Kanaya laboratory and also wishes to express all the other past and current members of Kanaya laboratory.

Finally, the author expresses his sincere gratitude to his family.

March, 2008

Hiroki Ogawa 